\title{
AN AGENT-BASED COMPUTATIONAL MODEL FOR BANK FORMATION AND INTERBANK NETWORKS
}




\section{AN AGENT-BASED COMPUTATIONAL MODEL FOR BANK FORMATION AND INTERBANK NETWORKS}

By

OMNEIA R.H. ISMAIL, M.SC.

A Thesis Submitted to the

School of Graduate Studies

in Partial Fulfilment of the

Requirements for the Degree of

Doctor of Philosophy

McMaster University

(C)Copyright by Omneia R.H. Ismail, July 2012 
Doctor of Philosophy (2012)

McMaster University

Hamilton, Ontario

School of Computational Engineering and Science

TITLE: An agent-based computational model for bank formation and interbank networks

AUTHOR: Omneia R.H. Ismail, M.Sc. (Twente University/ McMaster University ) SUPERVISOR: Professor Matheus R. Grasselli

NUMBER OF PAGES: xiv, 173 


\begin{abstract}
The aim of this thesis is to study the role of banking in society and the effect of the interbank market on the performance of the banking system. It starts by reviewing several studies conducted on empirical banking networks and highlighting their salient features in the context of modern network theory. A simulated network resembling the characteristics documented in the empirical studies is then built and its resilience is analyzed with a particular emphasis in documenting the crucial role played by highly interconnected banks.

It is our belief that the study of systemic risk and contagion in a banking system is an integral part to the study of the economic role of banks themselves. Thus the current work focuses on the fundamentals of banking and aims at identifying the necessary drivers for a dynamical setup of the interbank market.

Through an agent-based model, we address the issues of bank formation, bank runs and the emergence of an interbank market. Starting with heterogeneous individuals, bank formation is viewed as an emergent phenomenon arising to meet the needs for investment opportunities in face of uncertain liquidity preferences. When banks work in isolation (no interbank market), in the long run and through a long experience with bank failures, banking turns into a monopoly or a market with few players.

By equipping banks with their own learning tools and allowing an interbank market to develop, fewer bank failures and a less concentrated banking system are witnessed. In addition, through a scenario analysis, it is demonstrated that allowing banks to interact does not weaken the banking system in almost all the cases, and improves the performance on multiple occasions.

The work is concluded by studying the effects of a banking system on individuals and the economy in what is called social measures. We establish that the effects of banking on social measures such as consumption level, consumption inequality between individuals, long term investment and economic waste, varies significantly based on the structure of the society.
\end{abstract}




\section{Acknowledgements}

To work on a PhD degree is a long journey, and coming to its end I would like to express my gratitude to a number of people who shaped my journey.

I have been very fortunate working with my supervisor Prof. Matheus Grasselli, who provided insightful guidance, critical questions throughout my years as his student, and invaluable help with the writing of this thesis. Working under his supervision I enjoyed the freedom as a student to think and to pursue my research interest. I am in debt to him for allowing me such freedom and for his patience with my wanderings throughout the work on this thesis.

I had the best committee members I could have hoped for: Profs. Tim Davidson and Tom Hurd. With time constrains, they kindly gave me the opportunity to work on my topic as an initial trial before judging its validity, then encouraged me as results materialized and crucially directed my path early on. I cannot thank them enough.

I would like to particularly thank Prof. Hurd, who over the years of my study at McMaster looked after me, guided me in instances defining my academic life, and always welcomed my academic existentialist discussions.

I would also like to thank Prof. Leigh Tesfatsion and Prof. Alfred Lehar for stimulating discussions.

I am grateful to many people who taught me over the years. My undergraduate teachers in Cairo university (Egypt) and my graduate teachers at Twente University (The Netherlands) and McMaster University. Especially I would like to thank Profs. David Earn and Zeinab Amin, who have been wonderful teachers and influenced me far beyond the classroom.

I would also like to thank the faculty and staff back home at Cairo University for their continuous support and the staff at Twente University and McMaster, both at the Mathematics and Statistics department and the School of Computational Engineering and Science, for assisting me in many different ways.

I have been surrounded by the love and support of many close people, and I cannot 
imagine these past years without them. My coffee mates Pam and Sheila, my close friends Rosa Assalone, Dafna Nitzan and Tsunehiro Tsujimoto. My companion Nima Anvari with the calm and happiness he brought to my life. My very best friend since middle school Hoda Mira and my strong tie to home who always ended her emails with "I miss you so much". My small and loving family, Mourad, Iman and Adam. My guardian angel Ming Yan on the other side of the universe as the only person who could always see the light at the end of my PhD tunnel.

Finally my dearest people, my parents who I left almost a decade ago to pursue my graduate studies. They taught me that seeking knowledge comes first and have been my ideals. This thesis is the fruit of our time apart (I hope it announces an end to it!) and makes them proud. To them I dedicate it. 
To my parents

Salwa Abdullah, Raouf Hamed

and the times we have been together 


\section{Contents}

Abstract ................................ ii

Acknowledgements ....................... iv

1 Introduction 1

2 Networks $\quad 11$

2.1 Basic notions . . . . . . . . . . . . . . . . . . 11

2.1.1 Degrees ........................... 12

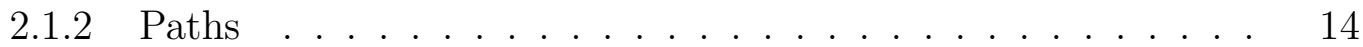

2.1.3 Clustering . . . . . . . . . . . . . . 16

2.1.4 Small-world effect . . . . . . . . . . . . . . . . . 16

2.1.5 Degree distributions . . . . . . . . . . . . . . . 17

2.2 Network generating models . . . . . . . . . . . . . . . . . . . . . . 18

2.2.1 Erdős-Rényi model . . . . . . . . . . . . . . . . . . 18

2.2.2 Small world model . . . . . . . . . . . . . . . . . . . . . . . . 19

2.2.3 Preferential attachment . . . . . . . . . . . . . 20

2.3 Scale-free networks . . . . . . . . . . . . . . . . . . . . . 21

2.3.1 Generating Model . . . . . . . . . . . . . . 21

2.3.2 Failures and attacks .............. . . 25

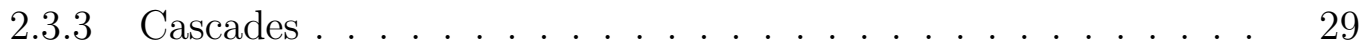

3 Empirical Networks $\quad 34$ 


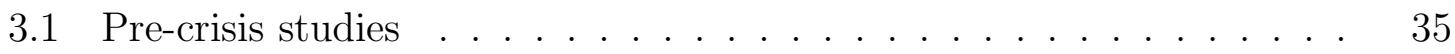

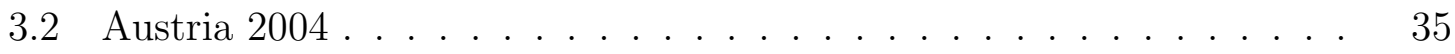

$3.2 .1 \quad$ Japan $2004 \ldots \ldots \ldots \ldots$

$3.2 .2 \quad$ USA $2007 \ldots \ldots \ldots \ldots \ldots \ldots$

$3.2 .3 \quad$ Brazil $2007 \ldots \ldots \ldots \ldots$

3.3 Post-crisis studies . . . . . . . . . . . . . . . . . . . . . . . . . . . . 39

$3.3 .1 \quad$ Italy $2009 \ldots \ldots \ldots \ldots$

$3.3 .2 \quad$ Brazil $2009 \ldots \ldots \ldots$

4 Simulated networks $\quad 46$

4.1 Network construction . . . . . . . . . . . . . . . . . . . . 46

4.1.1 Preferential attachment in a directed unweighted network . . 47

4.1 .2 Examining the simulated network . . . . . . . . . . . 52

4.1 .3 Directed weighted network . . . . . . . . . . . . . . 55

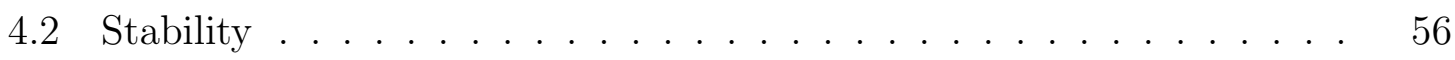

4.2.1 Single bank defaults in scale-free banking networks . . . . . 57

4.2 .2 Single bank defaults in large networks . . . . . . . . . . 60

4.2.3 Highly connected banks in a scale-free interbank network. . . 65

4.2.4 The Systemic Risk Index . . . . . . . . . . . . . . . . 66

4.3 Conclusions and further directions . . . . . . . . . . . . . 68

5 Bank formation and bank runs $\quad 71$

5.1 The Diamond and Dybvig Model . . . . . . . . . . . . . 71

5.2 The pre-banking society $\ldots \ldots \ldots \ldots \ldots \ldots \ldots$

5.2.1 Individuals, preferences, and investment choices . . . . . . 75

5.2 .2 Searching for partners $\ldots \ldots \ldots \ldots \ldots$

5.2 .3 Learning and predicting . . . . . . . . . . . 78

5.3 Bank formation . . . . . . . . . . . . . . . . . . . . . 81

5.3 .1 Joining a bank . . . . . . . . . . . . . . 81 
$5.3 .2 \quad$ Bank birth . . . . . . . . . . . . . . . . . 84

5.4 Bank runs . . . . . . . . . . . . . . . . . . . . . . . 86

5.4 .1 Bank survival problem . . . . . . . . . . . . 87

5.4 .2 Banks learning and allocation problem . . . . . . . . 89

5.4 .3 Extensions . . . . . . . . . . . . . . . . . . . . . . 92

6 Interbank Market $\quad 94$

6.1 The Allen and Gale Model . . . . . . . . . . . . . . . . . . 95

6.2 Banks and learning . . . . . . . . . . . . . . . 100

6.2 .1 Learning from experience . . . . . . . . . . . . . . . 100

6.2.2 Windows of opportunities . . . . . . . . . . . . . 102

6.2 .3 Desired deposits . . . . . . . . . . . . . . . . . . . 103

6.3 Building the network . . . . . . . . . . . . . . 105

6.3.1 Preferential attachment . . . . . . . . . . . . . . 106

6.3 .2 Dissolving links . . . . . . . . . . . . . . . . . . 107

6.4 Numerical experiments . . . . . . . . . . . . . . . . . . . . . . 113

6.4 .1 Scenario analysis . . . . . . . . . . . . . . . . 117

6.5 Correlated preference shocks . . . . . . . . . . . . . . . . 122

6.5 .1 Preference regions . . . . . . . . . . . . . . . . . 122

6.5.2 Numerical experiments . . . . . . . . . . . . . . . . . . 125

6.6 Conclusions . . . . . . . . . . . . . . . . . . . . . . . . . . . . . . . . . 131

7 Banks and Social Measures 132

7.1 Satisfaction . . . . . . . . . . . . . . . . . . . . 133

7.1.1 Accumulation and redistribution . . . . . . . . . . 135

7.1.2 Differences between individuals . . . . . . . . . . . . 138

7.1.3 Numerical experiments . . . . . . . . . . . . . . . . . . . . . 139

7.2 Consumption, investment and waste . . . . . . . . . . . . . . 144

7.2.1 Individual consumption, inequality, and the cost of banking . 144 
7.2 .2 Investments and liquidations . . . . . . . . . . . . 146

7.2 .3 Further trends . . . . . . . . . . . . . . . . . . . . . . . . . . . 148

7.3 Consumption and investment in societies with correlated preference shocks . . . . . . . . . . . . . . . . . . . 154

7.3.1 Correlated periodic preference shocks . . . . . . . . . . 154

7.3 .2 Communities . . . . . . . . . . . . . . . . 157

7.3 .3 Further trends . . . . . . . . . . . . . . . . . . . . 160

7.4 Conclusions . . . . . . . . . . . . . . . . . . . . . . 162

8 Conclusions and Further Directions 165

$\begin{array}{ll}\text { A Numerical Experiments } & 168\end{array}$

A.1 Banking system performance: interbank vs no interbank . . . . . 168

A.2 Effects of banking on social measures . . . . . . . . . . . . . 169 


\section{List of Figures}

4.1 Network for $\alpha=0.05 \ldots \ldots \ldots \ldots \ldots \ldots \ldots$

$4.2 \quad$ Network for $\alpha=0.1 \ldots \ldots \ldots \ldots \ldots \ldots \ldots \ldots$

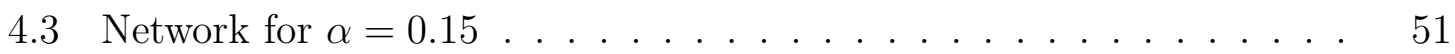

4.4 Histograms for the in and out-degree distributions for different $\alpha$ (attachment probabilities) values. . . . . . . . . . . . . . . . 52

4.5 Histogram of $D I$ caused by a single bank default in a scale-free network. 57

4.6 Histogram of the ratio of the total capitalization lost to the defaulted bank capitalization in a scale-free network. . . . . . . . . . . 58

4.7 Relation between number of the defaulted bank in-degrees and the number of subsequent defaults in a scale-free network. . . . . . . . 59

4.8 Relation between in-degrees of defaulted banks and the percentage of capitalization lost in a scale-free network. . . . . . . . . . . . . 59

4.9 Histogram of banks in-degree for scale-free and random networks. . . 61

4.10 Histogram of default impact of a single bank default in scale-free and random networks. . . . . . . . . . . . . . . . . . . 62

4.11 Relation between the in-degree of initially defaulted bank and the number subsequent defaults in scale-free (top) and random (bottom) net-

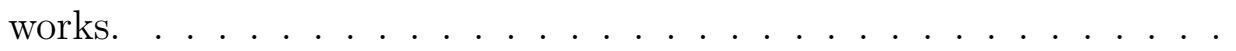

4.12 Relation between the in-degree of initially defaulted bank and default impact in scale-free (top) and random (bottom) networks. . . . . . 
4.13 Results for 500 Simulated Networks. Top 10 connected banks compared to rest of the banks in a scale-free network of 500. . . . . . . . . . 65

4.14 Results for 500 simulated networks, in a 1000 Monte Carlo runs. . . . 67

5.1 Society, preference shock, and the search for partners. . . . . . . . 78

5.2 Percentage of correct predictions. . . . . . . . . . . . . . 80

5.3 Banks established after 80 periods in a society of 6400 individuals, without the possibility of a bank run. . . . . . . . . . 86

5.4 Banks evolution in 100x100 society over the course of 100 periods . . 91

6.1 Banks learning, an example . . . . . . . . . . . . . . 103

6.2 Cyclicity in interbank at time $t=2$, an example . . . . . . . . . 109

6.3 Established banks in a $100 \times 100$ society over the course of 100 periods with interbank market . . . . . . . . . . . . . . . . . 114

6.4 Histogram of number of banks established by the end of the 80-th period for the 50 simulations, interbank case (upper graph) and no interbank case (lower graph). . . . . . . . . . . . . . . 115

6.5 Communities established in a $100 \times 100$ society. . . . . . . . . . . . . 124

6.6 Histogram of number of banks established by the end of the 80-th period for the 50 simulations, interbank case (upper graph) and no interbank case (lower graph). Preference shocks every 15 periods. . . 126

6.7 Histogram of number of banks established by the end of the 80-th period for the 50 simulations, interbank case (upper graph) and no interbank case (lower graph). Preference shocks every other period. .

6.8 Histogram of number of banks established by the end of the 80-th period for the 50 simulations, interbank case (upper graph) and no interbank case (lower graph). Established preference communities. . . 130

7.1 Accumulated deviations in a $80 \times 80$ society with no banks by the end of 80 periods. . . . . . . . . . . . . . . . . . 136 
7.2 Accumulated deviations in $80 \times 80$ society with banks (No interbank market) by the end of 80 periods. . . . . . . . . . . . . . . . 137

7.3 Accumulated deviations in $80 \times 80$ society with banks (interbank market) by the end of 80 periods. . . . . . . . . . . . . . . . . 137

7.4 Evolution of Gini index over time in a $80 \times 80$ society over the course of 80 periods. . . . . . . . . . . . . . . . . . . . . . 139

7.5 Evolution of Gini index over time for societies with no banks - 50 independent simulations of $80 \times 80$ society over the course of 80 periods. 140

7.6 Evolution of Gini index over time for societies with banks yet no interbank market -50 independent simulations of $80 \times 80$ society over the course of 80 periods. . . . . . . . . . . . . . .

7.7 Evolution of Gini index over time for societies with banks and interbank market -50 independent simulations of $80 \times 80$ society over the course of 80 periods. . . . . . . . . . . . . . . . . .

7.8 Gini index, percentage of thrilled individuals, and banking coverage for the 50 independent simulations of societies with banks with No interbank market (upper), and for societies with banks in interbank market (lower). . . . . . . . . . . . . . . . .

7.9 Mean of positive and negative deviations for the 50 independent simulations of societies with banks but no interbank market (upper), and societies with banks and interbank market (lower). . . . . . . . . . 143

7.10 Individuals' consumption Gini index and their relative aggregate consumption relative to the sum of the consumption together with banks reserves at the end of the 80th period for the 50 independent simulations of society with banks with No interbank market (upper), and for banks with interbank market (lower). . . . . . . . . . . . 
7.11 Gini index and the percentage of individuals not in banks at the end of the 80th period for the 50 independent simulations of society with banks with No interbank market (upper), and for banks with interbank market (lower). . . . . . . . . . . . . . . . . . . . . 150

7.12 Individuals' consumption Gini index and banks aggregate reserves at the end of the 80th period for the 50 independent simulations of society with banks with No interbank market (upper), and for banks with interbank market (lower). . . . . . . . . . . . . . . . . . . . . . 152

7.13 Aggregate individuals' consumption and banks aggregate reserves at the end of the 80th period for the 50 independent simulations of society with banks and no interbank market (upper), and for societies with banks in the interbank case (lower) . . . . . . . . . . . . 153 


\section{List of Tables}

4.1 Parameters values for choice of attachment probability $\alpha \ldots \ldots$. . . 49

4.2 Parameters estimated for power law fitting of the simulated networks. $\quad 55$

6.1 Regional liquidity shocks . . . . . . . . . . . . . . . . . 96

6.2 Networks topology considered in Allen and Gale . . . . . . . . . . 97

6.3 Regional liquidity shocks with perturbation . . . . . . . . . . . 98

6.4 Dissolving Cyclicity in the interbank market at time $t_{2 k+2}$ in a network with one cycle. . . . . . . . . . . . . . . . . . . . 111

6.5 Dissolving cyclicity in Interbank at time 2, another example . . . . . 112

6.6 Dissolving cyclicity in the interbank market at time $t_{2 k+2}$ in a network with three interconnected cycles. . . . . . . . . . . . . . 113 


\section{Chapter 1}

\section{Introduction}

Motivated by the recent financial crisis, there has been increased interest in the study of banking systems. With the unfolding of the crisis, many concerns were raised that called for considering additional factors that can contribute to the stability of the system and were not sufficiently accounted for in the standard theory.

Human causes and panic responses were argued to be the core of the recent crisis, in a clear manifestation of "tragedy of the commons", where self-interested individual actions cause a system wide failure. Too much government in the form of incentives in affordable housing programs, or too little in the form of lax regulation, were alternatively said to weaken the resilience of the financial system [Hal09]. Questions were raised about the validity of the assumptions underlying the models used by financial institutions, such as normality of distributions, rationality of market players, continuous price changes, etc. Each of these assumptions had been discussed before, together with proposed modifications [MH04]. Yet with the aftermath of the crisis, the criticism of these assumptions was grounded in the fact that they collectively reflected a picture of a manageable financial system with modest price changes that clearly underestimated the probabilities of such crises. Indeed, even as the first signs of crisis started to unfold, many held the believe that the odds of a system wide crisis were still too low to be of any importance. 
Another concern was the structure of the modern financial market. It was argued that financial innovation and integration have produced a highly interconnected complex financial network that further weakened the financial systems exposing it to what can be referred to as a "domino effect", where the collapse of one of the key "hubs" of the network caused a collapse of further links exposing the whole system to a systemic crisis. The effect of financial innovations and interbank links on the stability of the financial system had long been considered in literature [Oor90] with two opposing viewpoints. The first is that they can work as a propagation mechanism of small shocks and eventually cause a crisis to the whole system. For example in Gai, Jenkinson and Kapadia [GJK07] it is held that even though financial innovation and integration had served to make systemic crises less likely than in the past, the impact once occurred would have a significantly larger scale. That view is clearly expressed in the crisis aftermath paper by Haldane [Hal09]. On the other hand there is the view which expresses the initially held belief that innovation and integration work to spread the risks inherent in the financial system and improve liquidity, as expressed by Shiller in [Shi08], as well as by many earlier research papers arguing that a more complete set of links between banks would lower the risk of system contagion [AB08].

This last concern was particularly motivated by the recent advances in network theory. Network thinking, that is, focusing on relationships between different entities as the unit of study as well as the entities themselves, provided a novel language in describing many complex systems, particularly those where the complexity arises from the interaction among the system units in addition to the anatomy of their connection network. In late 90s the publication of two papers by Watts and Strogatz [DJW98] and Barabási and Albert [BA99] motivated a wide interest in networks and the search for unifying principles governing the networks found in different branches of science from earthquakes, ecosystems, power transition networks, gene networks, to the spread of infectious diseases [Mit09]. Under the new direction an emphasis has been given to how the network anatomy affects the network dynamical behaviour and 
its stability, and paved the way to see similarities between networks in different fields of study. For example, Haldane's paper [Hal09] draws similarities between the SARS epidemic, HIV/AIDS prevention programs, fisheries management, and the current crisis, and argues that they all represent environments exposed to risk that can be studied under the dynamic network framework.

The literature addressing banking networks can generally be classified into two streams. The first, and more recent, is based on empirical studies that typically start with a certain assumption on the topology of actual banking networks and proceed to examine their stability. Within this stream, supported by multiple empirical studies for actual financial networks (US, Austria, Italy, Brazil among others countries), the current trend motivated by recent work in network theory is to start from so-called scale-free network of banks and impose a shock (endogenously or exogenously), observing the effect on the network in terms of observed losses. Such approach emphasizes the interconnected nature of the network, in particular the notion of robust-yet-fragile systems, that is, robust with respect to random shocks affecting all of its nodes but fragile with respect to targeted failures of important banks.

The second stream of literature, on the other hand, bears a more theoretical flavour. It starts with a simplified model with typically a small number of homogeneous banks and examines the interaction and stability of this small network under a variety of theoretical scenarios for shocks.

Based on their nature, the two streams essentially targeted different questions and as such on multiple occasions can be viewed as drawing different conclusions. Amongst them, there are four major questions to be addressed for a full understanding of the banking system:

1. why was the network initially formed?

2. what are its current properties?

3. how do these properties affect its functionality and stability? 
4. how does the network evolve over time?

The first question of how the network came about and what motivated banks to interact in an interbank network, we believe to be better addressed in the theoretical stream set of studies, while the second question concerning the current shape and structure of the network is better addressed in the empirically based studies. The third question of the effect of network topology on its stability has been addressed by both streams of literature, nevertheless leading to what might be viewed as contradictory conclusions. The recent stream of empirical-based studies documented the network topology as being scale-free and accordingly concluded the robust-yet-fragile nature of the network (see for example [CS09] on the Brazilian interbank market). On the other hand, theoretically based studies addressing network and the effect of topology on its stability told a different story where the more connected the interbank network is the more stable it is. For example, the well-cited paper of Allen and Gale [AG98] supported the idea that connected complete networks strengthened the financial systems by spreading any losses that might be suffered from a single bank throughout the network, thereby lowering the possibility of any systemic risk. The fourth question, regarding the evolution of the network, is rarely addressed, apart from a few empirical studies describing how the network characteristics change using several snapshots of the system at different points in time [AB08].

Differences in results can be attributed to different mechanisms for propagation of shocks in the banking network. The Allen and Gale model (which will be denoted by AG) considered shocks as an excess liquidity demand faced by single banks based on the Diamond and Dybvig model [DD00] (denoted by DD) and used the mechanism of direct interbank linkages as the source of contagion in the financial system. Similar models are found in Temzelides [Tem97], Dasgupta [Das04], and Romero [Rom09], except that the propagation of the shocks is mainly carried out by agents in the system rather than by direct transfer through interbank links. For example, [Rom09] considers a social network of agents, where the agent decides on early withdrawing 
by asking three of his eight neighbours if they have already withdrawn.

On another strand we find papers like Cifuentes, Ferrucci and Shin [CFS05] and Nier et al [NYYA07], which consider models of systemic risk yet are not based on an initial shock through bank runs as in the previous papers. In [CFS05], there are a number of banks with direct interbank linkages and the authors are interested in examining the effect of changes in asset prices as a single bank failure tracks the sale of assets and a fall in the asset prices, coupled with the mark-to-market regulation and minimum capital ratio requirements. On the other hand in [NYYA07], a simulation based work, the authors generate a network of a number of banks guided by a bank-level, as well as aggregate-level, balance sheet restrictions, and an initial shock targeted to the asset side of the bank wipes out a certain percentage of the bank's external assets. In the two papers the authors report a nonmonotonic relation between the interbank network connectivity and system resilience to contagious default. In [CFS05] the contagion effect is small either when there are no interbank connections or when every bank is connected to every other bank. Similarly in [NYYA07] an initially small increase in connectivity between banks increases the contagion effect, nevertheless after a threshold value the connectivity improves the system resilience to contagion effects. The authors also reported that for less capitalized systems an increase in connectivity is associated with an increase in contagious defaults, and all else being equal, the more concentrated the banking system is the more prone to systemic breakdown.

Apart from the seemingly contradicting results drawn from different streams of studies, researchers have been under pressure (see for example [SLU09], [MLS08] and [Hal09]) to employ the recent advances in network theory and fill the gap between the financial systemic risk studies and some other fields of study such as epidemiology and ecosystems, which routinely use concepts such as the "small-world effect" (six degrees of separation principle), "scale-free" feature (most nodes have very few link connections and very few nodes have many connections), and "Achilles heel" (robust 
yet fragile network).

Such calls for employing recent advances of network theory in studying banking system need to be answered with a careful consideration of the limitations in the empirically based stream of studies and with a critical appreciation of the insights offered by the theoretically based stream. Empirically based studies, while based on actual network structures as a starting point in examining the system stability, provided a rather static picture of the system as manifested by knocking one bank at a time and examining the domino effect, if any, on the network. Such an approach, while providing the flexibility of examining the network, neglected the dynamical nature of the system where banks as well as individual depositors affect each other, learn, adapt and interact. In addition, the approach lacked the motivating forces for the dynamical nature of the system, such as the reasons for bank failures or the motives for banks to interact with each other. Rather the approach imposed a set of simplifying assumptions to compensate for the lack of such forces, such as the mechanical nature of bank failures by knocking them down and the zero recovery rates associated with their failure.

On the other hand we find that theoretical studies such as that of Allen and Gale [AG98], by employing an economist's intuition in addressing bank failures and bank interaction, provided the interacting forces that the empirical based studies lacked. Nevertheless, theoretical studies building on assumptions of optimized behaviour as well as restricting network topologies hindered their generalization to more relevant settings or different network structures. Between the static picture provided by the empirically based studies and the simplified and constrained picture of the theoretical studies, the underlying theory of banking systems implicitly or explicitly reflected an unrealistic picture of a manageable and predictable organization. We believe the more appropriate call for the banking theory is to combine the contributions of both streams, the flexibility of the empirical based studies and the quest to understand the underlying dynamical forces in theoretically based studies, in a new direction of 
Omneia R.H. Ismail - PhD Thesis - McMaster University - CES

research.

Two core modifications to the assumptions of orthodox economic theory have recently been proposed, helping pave the way for such new directions of research. The first is an emphasis on inductive rather than deductive thinking. The deductive approach for economic analysis has long been argued against by many economists given that our rationality is essentially bounded (the notion of bounded rationality goes back to Nobel prize laureate Herbert Simon in the 1950s [Sim55]). Recently, it has been explicitly argued that inductive reasoning (as put forward in the El Farol problem by Arthur [Art94]) gives a proper candidate approach or model for our decision making. That approach implied a learning, adaptive process through which market participants form hypotheses given their set of information and experiences, test the hypotheses, learn and update their beliefs accordingly. The second modification is a rejection of the micro foundations for macroeconomic behaviour, namely aggregating micro level components to reach the macro level together with the notation of the "representative agent" in finance and economics. The recent study of chaos gave the flavour of complexity to many disciplines including economics, together with the notion of emergence giving a clear distinction between the micro and macro level interactions, where the interaction between micro level components can lead to complex macro level phenomena that are not deducible from our knowledge about the components.

These two modifications imply a new approach of doing analysis, as manifested in the rise of bottom-up modelling to capture emergent phenomena coupled with relying on simulations of artificial adaptive agents employing inductive thinking within their given environments. The result is the new discipline of agent-based modelling in economics $(\mathrm{ABM})$, reflecting the view of considering the economy as an evolving complex system. The goal of ABM is not only to capture the dynamical nature of a system but also its emerging properties. Since its introduction in early 1990s, ABM had been applied to many economical problems, such as the building of artificial finan- 
cial markets (one of the most famous is the Santa Fe artificial financial market) that worked successfully in solving many empirical puzzles, such as fat-tailed distributions of returns and volatility clustering.

Combining the basic ideas of the ABM with network analysis, we believe would land us somewhere in the domain of adaptive coevolutionary networks, where there are the two forces of dynamics for the banking network: dynamics of relationships between the banks (the topology of the network itself as the unit of dynamics) and dynamics of the banks themselves as unit of analysis (the nodes in the network as the unit of dynamics while holding the topology static) [GB08].

Our aim is to study the effect of the interbank market on the performance of the banking system. Accordingly, we organize our work as follows. We start with a brief introduction to network theory in Chapter 2, mostly to fix the notation and explain the main concepts as applied to the interbank market. This chapter also addresses some of the widely discussed phenomenon of modern network models and presents three of the most well-known network generating models. We take a particular interest in the special kind of network known as scale free. We discuss the origin of the discovery of such topology, its characteristics and generating algorithms. We present the implications of such topology on the network resilience to shocks and the extreme case of cascading failures.

This is followed by a review of empirically observed characteristics of actual networks and examination of the effects of such structures on the system stability in Chapter 3. The set of studies all agree on reporting a scale-free nature in the different networks. We conclude the overview of empirical studies with a detailed presentation of the recent study carried for the Brazilian interbank market by Cont and Santos [CS09] and their methodology for quantifying contagion risk. Based on the observed characteristics we build a simulated network to mimic these characteristics in Chapter 4, upon which we examine network stability under the risk of contagion. In addition we examine the effect of network topology on its stability by considering different 
network topologies.

As indicated such empirically based studies are essentially static ones. Moving into a dynamic model constitutes the majority of the work in this thesis. Guided by the intuition presented in theoretical studies, we build a dynamical model of the interbank market and examine the effects of the interbank market on our system. Presenting an agent-based model for an interbank network, we address the issues of bank formation, bank run and interbank emergence in the banking system. Such a dynamical model, which by structure addresses different aspects of the interbank market and effects on the banking system, provides a complementary understanding of the banking system, rather than a contradictory one to that of the static model of empirically based studies.

In a simplified agent-based model, we position ourselves at the moment preceding the creation of the banking system and witness what Hayek referred to as the "simple power of organic growth" [Hay44] in our society. In a society which we do not deem predictable and far from manageable, we provide a simple view of the historical development of banks and the interbank market. Starting with heterogeneous individuals we view bank formation in Chapter 5 as an emergent property of the individual heterogeneous needs for investment opportunities. We then discuss what might motivate the establishment of banks, how they operate, and under what conditions they are subject to bank runs.

Our setup permits the establishment of multiple banks that further call for building an interbank market to safeguard against anticipated liquidity shocks. The possibilities of cooperation is examined in Chapter 6, together with a detailed discussion of how banks establish and dissolve interbank links. We then present how our model performs under such an interbank cooperation versus the case where banks are to operate in isolation from one another. The chapter is then concluded with examining the performance of our model under different parameter choices. Through a various set of Monte Carlo simulations we conclude that the presence of the interbank mar- 
ket leads to a better banking system as manifested with fewer bank runs and a less concentrated banking system.

In Chapter 7 we discuss the effects of banking and interbank market on the society welfare and its economic status. We introduce the idea of desire deviation and illustrate how banks contributed to a redistribution of satisfaction, as well as a significant change to the individuals consumption levels in the society. In addition, we examine the effects of banking on long term investment in productive technologies and economic waste.

Our model, in its simplicity, facilitated the examination of multiple core ideas in banking and upon which we are able to draw a number of interesting conclusions presented in Chapter 8. 


\section{Chapter 2}

\section{Networks}

In this chapter we first introduce the basic networks notions and terminology that will be used later and explain how they are related to the particular case of banking

network. At the second part of this chapter we address some of the widely discussed phenomenon of modern network models and present three of the most well-known network generating models.

\subsection{Basic notions}

Networks (also known as graphs in mathematical literature), are collections of nodes (vertices or sites) joined together by edges (links or bonds). For banking networks, the nodes are financial institutions and edges are established relations or interbank links between them, such as deposits, loans, or derivative exposures.

Graphs where more than one edge is allowed to connect two nodes are referred to as multigraphs and the edges are called multi-edges, whereas those where edges can connect vertices to themselves are called self-edge, or self-loops. Graphs that have neither self-loops nor multi-edges are called simple graphs or simple networks [New10], and will be our interest of study.

Networks can be represented in a number of ways, the most common is through 
the use of the adjacency matrix $A=\left(a_{i j}\right)$. Let us denote the number of vertices by $n$ and the number of edges connecting them by $m$. An adjacency matrix, is then an $n \times n$ matrix with elements

$$
a_{i j}= \begin{cases}1 & \text { if there is an edge between node } i \text { and } j \\ 0 & \text { otherwise }\end{cases}
$$

Accordingly, in a simple network we expect all the diagonal entries to be zeros (no loops), and the matrix to be symmetric (edge between $i$ and $j$ is the same as edge between $j$ and $i$ ).

Another character the edges might have is a direction, in which case the network is referred to as directed network, or digraph. Each edge has a direction pointing from one node to another, such as an interbank deposit from one bank into another, represented as lines with arrows in a graphical form. In such a case, the adjacency matrix would then be defined as

$$
a_{i j}= \begin{cases}1 & \text { if there is an edge from node } j \text { to node } i \\ 0 & \text { otherwise }\end{cases}
$$

Unlike the undirected case, this matrix would generally be asymmetric.

Edges can be carrying weights or strength (value to them), such as the size of the deposits in the case of banking networks, and we call the graph/network a weighted network in this case. Similarly, vertices can also have weights attached to them.

While the weights can be presented directly in the adjacency matrix $A$, we prefer for our analysis purpose to introduce a new matrix denoted by $L$ to carry the weights of the edges, either for the directed or undirected case.

\subsubsection{Degrees}

The degree of a node is the number of edges attached to it. In banking networks, the degree of a node (or a bank) is then the number of interbank links that the bank 
has. Let us denote the degree of node $i$ by $k_{i}$. Using the adjacency matrix, in an undirected network the degree can then be defined as:

$$
k_{i}=\sum_{j=1}^{n} a_{i j} .
$$

Given that we have $m$ edges, and each having two ends attached to different nodes, it follows that

$$
2 m=\sum_{i=1}^{n} k_{i}
$$

or

$$
m=\frac{1}{2} \sum_{i j} a_{i j} .
$$

As such the mean degree $\langle k\rangle$ of a vertex in an undirected network is given by

$$
\langle k\rangle=\frac{1}{n} \sum_{i=1}^{n} k_{i},
$$

which can be written as $c=\frac{2 m}{n}$

Another measure related to the mean degree in a simple network is the density $\rho$, which is defined as the ratio between the number of edges present in a network to the maximum possible number of edges $\left(\begin{array}{l}n \\ 2\end{array}\right)$ :

$$
\rho=\frac{m}{\left(\begin{array}{l}
n \\
2
\end{array}\right)}=\frac{\langle k\rangle}{n-1}
$$

The network is said to be dense if the ratio $\rho$ tends to a constant as $n \longrightarrow \infty$, whereas if that ratio tends to 0 the network is said to be sparse.

For the case of directed network, we have two additional measures for degrees. The first is the in-degree $k_{i}^{i n}$, defined as the number of incoming edges to vertex $i$. In banking networks, this is the number of interbank links corresponding to deposits that other banks have made with bank $i$. The second is the out-degree $k_{i}^{\text {out }}$, defined as 
the number of outgoing edges from vertex $i$. In banking networks, this is the number of deposits that bank $i$ has made in other banks. It then follows that

$$
\begin{aligned}
k_{i}^{\text {in }} & =\sum_{j=1}^{n} a_{i j}, \\
k_{j}^{\text {out }} & =\sum_{i=1}^{n} a_{i j} .
\end{aligned}
$$

The total number of degrees $k_{i}$ (total number of links of a vertex) would be the sum of the vertex in-degree and out-degree. The total number of edges $m$ is then the total number of incoming edges (or out-going edges):

$$
m=\sum_{i=1}^{n} k_{i}^{i n}=\sum_{j=1}^{n} k_{j}^{o u t}=\sum_{i j} a_{i j} .
$$

Similarly we have the mean in-degree $\left\langle k_{\text {in }}\right\rangle$ and mean out-degree $\left\langle k_{\text {out }}\right\rangle$ :

$$
\left\langle k_{\text {in }}\right\rangle=\frac{1}{n} \sum_{i=1}^{n} k_{i}^{\text {in }}=\frac{1}{n} \sum_{j=1}^{n} k_{j}^{\text {out }}=\left\langle k_{\text {out }}\right\rangle=\frac{m}{n} .
$$

\subsubsection{Paths}

A path is the sequence of pairs of vertices connected by an edge, the length of a path is then the number of edges traversed along the path, which can be calculated in a straight forward way using the elements of the adjacency matrix. The shortest path, also called geodesic path, is the path between two vertices such that no shorter path between them exists. On the other hand, the diameter of a network is the longest geodesic path between any pair of its vertices.

Denote the length of the geodesic path from vertex $i$ to $j$ by $d_{i j}$. The mean geodesic distance from $i$ to $j$, averaged over all vertices $j$ in the network is then

$$
l_{i}=\frac{1}{n} \sum_{j} d_{i j}
$$


A small mean distance for a vertex then can be used to infer the 'centrality' role of the vertex. As such we define the closeness centrality $C_{i}$ as the inverse of the mean geodesic distance:

$$
C_{i}=\frac{1}{l_{i}}
$$

If every vertex can be reached from any other vertex (i.e. there is a path connecting every and all vertices) we say the network is connected. There might be a pair of vertices with no paths between them, in which case we say that the network is disconnected. There may exist more than one path between a given pair of nodes. Two paths connecting a given pair are called edge-independent if they share no edges, and are called vertex-independent if they share no vertices. The number of independent paths between a pair of vertices in turn measures how strongly they are connected. Accordingly the number of independent paths between a pair of vertices is called the connectivity of the vertices.

A closely related notion is that of a component of a network, defined to be the subset of vertices that form a connected set. It follows that a connected network has only one component, while a disconnected network essentially has more than one component. Typically, there is a large component that constitutes most of the network (usually more than half) and is often referred to as the giant component, while the rest of the network consists of a number of small components disconnected from each other.

In a directed network, these notions become more complicated, since a path has a direction and accordingly a component can be expressed either as an in-component or an out-component.

Using these definitions, a commonly used criterion to measure network resilience is related to cut sets. A vertex cut set is the set of vertices whose removal will disconnect a specified pair of vertices. In case of banking networks, the notions of centrality, components, and cut sets play a key role in identifying the banks whose defaults yield a fragmented banking system. 


\subsubsection{Clustering}

Another important measure is the clustering coefficient, which is a measure of the network transitivity or 'local' connections density. It is defined as the probability that two vertices are connected to each other given that each of them is connected to a common third one (commonly corresponding to 'the friend of my friend is also my friend'). In banking networks this is identified as the probability that two banks have deposits with each other given that each of them has an interbank link with a third common bank.

In general, this can be expressed as a triangle, or a loop of length three. As such the clustering coefficient is defined as the total number of vertices forming triangles divided by the total number of triplets (i.e three connected vertices). In addition, clustering can be defined for single vertices. The clustering coefficient for vertex $i$ (and referred to as 'local clustering') is defined as

number of pairs of neighbors of vertex $i$ that are connected total number of pairs of neighbors of $i$

\subsubsection{Small-world effect}

The small-world effect (also known as "six degrees of separation"), refers to the surprisingly short distance between vertices in a network. The feature is attributed to Milgram's experiment [AB09] of distributing letters to hundreds of random people aiming at reaching a particular person by sending the letter to another person they know on a first name basis. The question was how many steps it would take for the letter to reach the destination. The answer came to be only about six steps. Putting it mathematically, it refers to a small mean distance,

$$
l=\frac{1}{n} \sum_{i} l_{i},
$$


between the vertices in a network. The wide use of the term arises from the profound implications of the phenomenon in networks coupled with the universality of its existence when examining actual networks. The effect is crucially reflected in banking networks as 'bad news for one bank being not too far off other banks'.

\subsubsection{Degree distributions}

One of the most frequently used property of networks is the frequency distribution of the vertices. Let $p_{k}$ denote the fraction of vertices in the network having degree $k$. It follows that $p_{k}$ then represents the degree distribution of the network. For a directed network, as to be expected, there are two distributions, the in-degree distribution and out-degree distribution. Even more informative is the joint distribution of in and out-degrees $p_{j k}$, that is, the fraction of vertices having in-degree $j$ and out-degree $k$.

A frequently reported distribution of vertices in real networks is a power-law distribution, as will be discussed in coming chapters of actual banking networks. This gives rise to what is known as scale-free networks, which are visually realized as we plot the degree distribution on a logarithmic scale and obtain a straight line. The logarithmic degree distribution is then a linear function of degree $k$ :

$$
\ln p_{k}=-\alpha \ln k+b
$$

for the constants $\alpha$ and $b$, and accordingly

$$
p_{k}=\beta k^{-\alpha}
$$

for $\beta=e^{b}$ is another constant.

The constant $\alpha$ is known as the exponent of the power law and has been observed to typically take values in the range $[2,3]$. Nevertheless, it has been documented that the actual degree distribution of observed networks does not follow the power distribution for the entire range of $k$, but rather that there is a cutoff value of $k$ after 
Omneia R.H. Ismail - PhD Thesis - McMaster University - CES

which the distribution follows the power law. This indicates that the power law is observed in the tail of the distribution for larger values of $k$.

\section{$2.2 \quad$ Network generating models}

A related question to the description of a network is how it can be generated so as to mimic observed data. We briefly introduce three of the most well-known network generating models: the random graph model, the small world model and the preferential attachment model.

\subsubsection{Erdős-Rényi model}

Random graphs are network models in which a certain set of parameters are fixed values but the network is random in other aspects. One of the simplest models for generating random graphs consists of fixing both the number of vertices $n$ and the number of edges $m$ in advance and proceeding to connect the edges to the vertices by choosing a pair of vertices uniformly at random and then connecting them with an edge. The model is usually referred to as $G(n, m)$.

A slightly different model, and more widely studied, is the Erdős and Rényi model, denoted by $G(n, p)$, where the number of vertices is fixed and the probability of connecting them is also fixed at level $p$. Accordingly, we place an edge between each pair with an independent probability $p$, so that the number of edges is not fixed. In other words, fix a number $n$ of vertices, where each pair has the same probability of being connected by an edge. As such we are sampling every edge of the total number $\frac{n(n-1)}{2}$ of possible edges with probability $p$ [Cal07].

In such $n$-vertex Erdős-Rényi networks, a vertex is connected to the rest of the $(n-1)$ vertices with a fixed probability of $p$, so it follows that the probability to be connected to a particular set of $k$ vertices, and not to the remaining $(n-1-k)$ vertices, is $p^{k}(1-p)^{n-1-k}$, and there are $\left(\begin{array}{l}n-1 \\ k\end{array}\right)$ of such $k$ vertices. The probability of 
being connected to exactly $k$ vertices is then

$$
p_{k}=\left(\begin{array}{l}
n-1 \\
k
\end{array}\right) p^{k}(1-p)^{n-1-k},
$$

which is a binomial distribution. As $n \longrightarrow \infty$ the distribution accordingly approaches a Poisson distribution, so the model is also known as the Poisson random graph.

As the probability of any two vertices to be connected to a third one is fixed at $p$, it follows that the clustering coefficient is $C=p$. Since it can be shown that $p=\frac{\langle k\rangle}{n-1}$ where $\langle k\rangle$ denotes the mean degree, we have that

$$
C=\frac{\langle k\rangle}{n-1}
$$

While this model is one of the best studied and originally adopted for its simplicity, it has a number of problems that make it unrealistic to be used for real world applications. First as it can be seen that the clustering coefficient given above approaches 0 as the number of vertices approaches infinity. Even for a finite number of vertices the clustering coefficient of random graphs is sharply smaller than the actual high clustering coefficient observed in real world networks. Second, and most importantly, the shape of the degree distribution does not correspond to that observed in empirical networks, which are typically right skewed with a few number of vertices being highly connected "hubs", while most vertices have low degree.

\subsubsection{Small world model}

This was introduced by Watts and Strogatz in 1998 [Wat02b] with the motivation of understanding the origin of the small-world effect. They believed it depends on two contradicting forces: a large clustering coefficient (two friends of a common friend are more likely to know each other than two randomly chosen people), and the possibility to connect rather distant people to each other in only a few intermediates. Their goal was to tune a network between complete disorder (random graph), and complete 
order (lattice).

The solution was a model starting with vertices arranged on a one dimensional line bent around like a circle, each connected to $z$ vertices nearest to it and thus achieving a high clustering coefficient, unlike in the random graph theory. Nevertheless the result is a degree distribution with all vertices having the same degree (regular graph), leading to the more serious problem of having a network with "large world" instead. This issue can be solved by imposing a small-world effect through removing or rewiring edges from the circle to randomly chosen positions with probability $p$. Yet, similarly to the random graph model, the resulting degree distribution is not rightly-skewed matching observed empirical networks.

\subsubsection{Preferential attachment}

It is worth mentioning that while the Erdös-Rényi random graph model produces networks with unrealistic degree distributions, this shortcoming can be modified and random graphs with general degree distributions can actually be constructed. The most widely used procedure is called the configuration model. Nevertheless, a common shortcoming of random graph models is that their parameters are fixed exogenously, and while they can be modified to produce power-law degree distributions, they do not offer any insight to explain why actual networks exhibit power law distribution. The preferential attachment model developed by Barabási and Albert in 1999 [BA99] aims to address this issue.

The underlying idea was first introduced by the Nobel prize laureate Herbert Simon as early as 1955 to explain the size distribution of business firms [New10]. It is rooted on the principle that once people (or a nodes) become rich (or large), regardless of how they became to be rich, they have better chances of getting even richer compared to their poorer counterparts. The idea was later picked up by Price in 1970 [New10] in modelling the citation network. He proposed a model of network formation where newly appearing papers cites previous papers, which in turn are 
chosen with probability proportional to the number of citations they already have. Price's model was not well known outside the information science community. It was independently discovered by Barabási and Albert who proposed a simple model for generating a scale free undirected network (unlike Price's model which is a directed one) that produces a power law degree distribution with exponent 3 [New10].

There are two essences of the preferential attachment model for network formation: growth (vertices are added gradually) and preferential linking (vertices are chosen proportional to their degree to be connected with the new vertex). In the Barabási and Albert model vertices are added one by one and each vertex connects to a randomly chosen vertex proportional to its degree. The number of connections for each vertex is fixed to be equal the average degree $\langle k\rangle$. A number of modifications have been proposed to their basic model, such as the possibility of not only adding edges but also removing edges, addition of extra edges, introducing a non-linear preferential attachment mechanism, and so on and so forth.

\subsection{Scale-free networks}

Since the introduction of the Barabási-Albert model, a vast amount of work has been carried out to study scale-free networks, either by studying more general mechanisms to construct such networks or by studying their properties, particularly those related to their stability in the face of shocks. Before going on to study the interbank market represented as scale-free networks, we aim first to shed more light into their nature and stability.

\subsubsection{Generating Model}

As addressed by Barabási and Albert [BA99], the origin of the observed power-law degree distribution in real networks has been attributed to two mechanisms: growth and preferential attachment. Their algorithm proceeds as follows: 
1. (initial state): Start the network with a small number of nodes $m_{0}$.

2. (growth): At every time step, add a new node with $m \leq m_{0}$ edges.

3. (preferential attachment): Link the newly added node to $m$ different existing nodes in such a way that the probability $\pi$ that a new node is connected to an existing node $i$ with degree $k_{i}$ is

$$
\pi\left(k_{i}\right)=\frac{k_{i}}{\sum_{j} k_{j}} .
$$

In terms of banking networks, this corresponds to starting with a few initial banks and at each time step add one bank to the system connected to these previously establish banks with probability based on how many interbank links these old banks already have.

Their algorithm results in a network with $t+m_{0}$ number of nodes after $t$ time steps, and $m t$ edges. It can be shown that it produces a network with a power-law degree distribution of exponent 3. In addition, the authors showed in a subsequent paper [AB02] that both the mechanisms of growth and preferential attachment together are 'simultaneously' responsible for the scale-free nature of the network, and not one mechanism without the other.

Before moving to more generalized models for generating scale-free networks, it is worth stating the properties of the networks produced by the Barabási and Albert model:

- Average path length: they showed that the average path length increases approximately logarithmically with the number of nodes $n$, and is always smaller than that of an Erdős-Rényi random network with the same number of nodes.

- Degree correlations: unlike Erdős-Rényi random networks where the node degrees are uncorrelated, their model produces networks where correlations between the degree of connected nodes develop spontaneously. 
- Clustering Coefficient: the clustering coefficient of their scale-free network is five times higher than that found in an Erdös-Rényi random graph. Although there is no analytical prediction of the clustering coefficient for their model, it can be shown to decrease with the network size following a power law

$$
C \sim n^{-0.75}
$$

compared to a decay of

$$
C=\langle k\rangle n^{-1}
$$

for an Erdős-Rényi random graph with $\langle k\rangle$ denoting average degree.

As indicated, the basic features of their model is producing a scale-free unweighted undirected network having a power-law degree distribution with a fixed predetermined exponent of 3 , relying on a linear simple preferential attachment mechanism and a constant addition of nodes, one at each time step. Further work has been introduced to generalize their model features to address other observed features of real networks, such as the observed exponential cutoff in the degree distribution. In what follows we consider some of the modifications and generalizations proposed in [AB02].

In the Barabási-Albert model, the growth of the network is linear in time (i.e., constant average degree), whereas when considering real networks it is observed that in many cases the average degree increases in time. This feature is referred to as 'accelerated growth', and happens when the number of edges (say interbank links) grows faster than the number of nodes (say banks). A number of modifications have been introduced to adjust for this observed feature where, in addition to adding a new node as a function of time, there is also the addition of new edges between previously existing nodes.

Real networks for which growth can be observed (e.g., actor collaboration network, citation network of articles) tend to exhibit a preferential attachment feature. Whereas for some of these networks $\pi(k)$ is observed to be linear in $k$, as assumed in 
Barabási-Albert model, in general it appears to follow the more general form

$$
\pi(k) \sim k^{\alpha}
$$

Nevertheless, according to the analysis of Krapivsky, Render and Leyvraz (as discussed in $[\mathrm{AB} 02])$, the scale-free nature of a network is destroyed under nonlinear preferential attachment. The only case where the scale-free feature is not destroyed is for an asymptotically linear preferential attachment.

An additional characteristic of the preferential attachment is the initial attractiveness of the node. As observed in Barabási-Albert model, a node with no links has an attractiveness (probability of getting edges) $\pi(0)=0$, whereas for real networks that is not the case, where nodes with no edges still can acquire edges. This leads to proposing the more general form of

$$
\pi(k)=A_{0}+k^{\alpha}
$$

having $A_{0}$ representing an initial attractiveness of node $i$. It is shown that such a linear shift in $\pi(k)$ in the case of linear preferential attachement does not destroy the scale-free nature of the network, but only changes the distribution degree exponent [AB02].

Along these modifications to the two basic features of the Barabási-Albert model, other modifications have been considered, among which:

- introducing local events that incorporate the addition/removal of nodes and the addition/removal of edges, in addition to an edges rewiring process;

- growth constraints, where some nodes or edges have a finite life/capacity. For example, banks can be thought of as having limited capacity of accepting deposits or limited resources to deposit with other banks; 
- embedding nodes with a competitive aspect of individuality, with each node being assigned a fitness parameter $\eta_{i}$, and modifying the preferential attachment probability to account for this fitness according to

$$
\pi_{i}=\frac{\eta_{i} k_{i}}{\sum_{j} \eta_{j} k_{j}}
$$

As such, even a relatively young node with few edges can still attract more edges at a higher rate than other noes due to its fitness parameter, a feature that is commonly observed in real world applications. In the case of banking networks, such competitive aspects can be thought of as banking credit rating for example.

\subsubsection{Failures and attacks}

The recently recognized nature of power-law degree distributions in real networks revived a vast interest in networks and their representations as scale-free ones. In addition, the implication of such configurations in terms of network resilience is a particularly intriguing topic.

Many complex real systems proved to be robust in the face of errors, where local failures in the system rarely lead to a system wide crisis. This stability has been commonly attributed to the redundant connections between the system underlying network components. In 2000, Albert, Jeong and Barabási [AJB00] demonstrated that this robustness to random failures is only displayed in one class of complex systems, namely those that can be characterized as scale free. Because of its significant contributions to the study of network resilience, we now highlight the results of their paper.

Complex networks can generally be divided into two types according to their degree distribution. The first is the well studied case of random graphs characterized by a degree distribution $p(k)$ peaked around the its average $\langle k\rangle$ which decays expo- 
nentially for large $k$. The most famous is the model of Erdös and Rényi which leads to a homogeneous network where each node has approximately the same number of links. The second type are scale-free networks, where the connectivity distribution is described by a power law distribution. Unlike the previous case, it is an inhomogeneous network with the probability of nodes with high links statistically significantly different from zero. In comparing their robustness, Albert, Jeong and Barabási constructed two networks with an equal number of nodes: a random network, that is, a Erdös-Rényi random graph $(n, p)$, and a scale-free graph. They then examined the effect of two types of node removal: a random node removal, where the node to be removed is chosen at random (an error or failure of a node), and a removal of nodes chosen according to their degree, such as removing the most connected nodes (an attack). The effect on the network was then examined using its diameter and giant components.

Recall that the diameter is defined as the average length of the shortest paths connecting any two nodes and reflects the nodes' ability to communicate with one another. The effect of removal of nodes on the network diameter can be summarized as follows:

- Error tolerance (random removal of nodes): when a small fraction $f$ of nodes is removed there is in general an observed increase in the diameter for both types of networks (random and scale free). For the random network the diameter increases monotonically with $f$, which can be easily explained in terms of the homogeneity of the network nodes: since all nodes have approximately the same number of edges the removal of each node causes the same amount of increase in the diameter. On the other hand we find a drastically different behaviour for the scale-free network: the diameter remained unchanged above a certain fraction $f$ (say $5 \%$ of node removal). This robustness to node failure is again attributed to the degree distribution of the nodes, where under the power law the majority of nodes have only a small number of edges. Thus nodes with small connectivity 
will be the one most likely selected under the random removal scenario. That in turn would not alter the network diameter all that much.

- Attack survivability (removal of most connected nodes): an 'informed' network attacker will target the most connected nodes of the network. To simulate such an attack the authors removed nodes in a decreasing order in terms of their degree starting with the most connected one. Since in the random graph the nodes have approximately the same number of connections, there is no witnessed difference between the previous scenario of random removal and the attack scenario. For scale-free networks, the situation is quite different, with the diameter increasing rapidly with each removal, and by the time of removing $5 \%$ of the most connected nodes the diameter is doubled. The scale-free network is thus vulnerable to attacks, and the removal of its highest connected nodes causes a drastic alteration of the network configuration.

Viewing a network as clusters of nodes, an outcome of removing a node is the process of cutting nodes from the main (i.e., giant) cluster. In other words, removing a node from a network means removing the edges this node has with other nodes, which in turn might cut these nodes from the giant cluster. Measuring the giant cluster as a fraction $S$ of the total network size (in terms of the number of nodes), the authors obtain the following:

- Error tolerance (random removal of nodes): in random graphs, when a fraction $f$ of nodes is removed, a threshold-like behaviour is observed, in which for a small $f$ there is no major change in $S$, yet as $f$ increases to reach a threshold $f_{c}$ (found to be $f_{c} \simeq 0.28$ in their case), the giant cluster falls into pieces and $S \simeq 0$. However, for the scale-free network, as expected, $S$ remains close to its is starting value, where the random removal of nodes causes only single nodes isolations, not the isolation of clusters of nodes (extremely delayed critical point). 
- Attack survivability (removal of most connected nodes): while the random graph displays the same behaviour under attach as that under error, the scale-free network again displays a different behaviour under the two scenarios. Unlike the case of random removal of node, in the attack scenario there is an early critical value $f_{c}$, smaller than that observed in random graph (observed to be $f_{c} \simeq 0.18$ ), above which the network breaks apart into isolated clusters.

From the above two measures, the authors concluded that scale-free networks display an exceptionally high degree of robustness (tolerance against random failures), nevertheless that tolerance comes at the expense of its attack vulnerability.

Following the work of Albert, Jeong and Barabási, an enormous interest has been devoted to study the robustness of scale-free networks. Crucitti et al. [CLMR04] examined the resilience of random and scale-free networks with respect to error and attack using three criteria for identifying the node importance: the degree of the node, the number of shortest paths that pass through the node, and the number of shortest paths recalculated every time a node is removed. Also they used a slightly different measure for effect of node removal on the network called global efficiency, defined as

$$
E(G)=\frac{1}{n(n-1)} \sum_{i \neq j \in G} \frac{1}{t_{i j}}
$$

for $t_{i j}$ is the shortest path length between nodes $i$ and $j$ in graph $G$. The measure is a modification of using the shortest path length (diameter), with the advantages that it is well-defined for non-connected networks and is a global property of the network.

Their results agree with those of Albert, Jeong and Barabási for the random removal of nodes and for the intentional attack on nodes with the highest degrees. On the other hand when considering the second and third criteria for choosing the important nodes to remove, they found that the results for the scale-free network do not substantially differ from these reported from choosing important nodes based on their degrees. The difference is more pronounced for the random graph case, where 
attacks based on a recalculated number of shortest paths cause a greater damage than that based on degree. They attributed these observations to the fact that in scalefree networks the nodes with the highest degree are also the nodes with the highest number of shortest paths, whereas for the random networks there is not a perfect parallel between them.

The above discussion of error and attack tolerance should not be confused with another closely related yet different topic of the cascading failures in complex networks. In our above discussion we examined the effect of node removal on the network connectivity, diameter or effectiveness, where it should be emphasized that upon the removal of the node, all its edges are also removed from the network. Then the resulting effect on the network is examined. The second class of models of cascading failures is addressing the effect of node removal or failure as the load on that failed node has to be rerouted to other nodes, which may eventually lead to an avalanche of overloads on other nodes that are not able to handle the extra load or traffic. This class of models is sometimes referred to as the dynamic approach of node failures, opposed to the static one represented earlier. The main mechanism responsible for a system-wide crisis rests on the process of load redistribution, a problem well comprehended in complex communication, transportation, electrical power grids systems and traffic networks. Under such consideration, it has been shown that a single node failure can collapse the entire system due to the dynamics of the redistribution of its loads.

\subsubsection{Cascades}

As we have just indicated, whereas the removal of the most connected links will affect the diameter of the network, the existence of a giant connected component does not depend on the presence of these highly connected nodes. In addition the giant connected component will still display the small-world feature after the removal of the highly connected nodes [ML02]. A more drastic effect is to be witnessed when 
the dynamic properties in terms of the flow of the network is examined, as opposed to the static properties presented so far.

In the paper by Motter and Lai [ML02] focusing on the cascades triggered by the removal of single node, they proposed a model where at each time step a single unit of information or flow is exchanged between every pair of nodes, and this information takes the shortest path between the two nodes. Define the load $L_{i}$ of a node $i$ as the total number of shortest paths going through it and the capacity of a node as the maximum amount of load the node can handle, which they proposed it to be a linear function of the initial load

$$
\text { Capacity }_{i}=(1+\alpha) L_{i}, \quad i=1,2, \cdots, N
$$

where $\alpha \geq 0$ is a tolerance parameter. Starting with a network of $N$-nodes, the removal of a node would trigger a change in the distribution of shortest paths, and accordingly of the load distribution, which might cause the failure of further nodes if their load exceeded their capacity. The process is called a cascade failure. The damage caused by such cascade is measured in terms of the change in largest connected component size:

$$
G=N^{\prime} / N
$$

where $N^{\prime}$ and $N$ are the number of nodes in the largest component after and before the cascade, respectively.

They then examined their model on the two types of networks, random and scale free, under the two scenarios of random removal of nodes and targeted attack of the highly loaded nodes. Under the scale-free network model they found that, for a random removal of nodes, on average $G$ remains close to unity. Yet under intentional attacks, there is significant reduction in $G$, where for a high value $\alpha=1$, the initial attack can reduce the size of $G$ by more than $20 \%$. The damage is larger for smaller values of $\alpha$ (for $\alpha=0.2$ the size of $\mathrm{G}$ is reduced by more than $60 \%$ ). 
The results are not as dramatic for the random graph model, where the system does not experience any cascade failure for $\alpha$ as small as 0.05, under either node removal scenario. The same amount of $\alpha$ though under the intentional removal of nodes for the scale-free network will reduce $G$ to less than $10 \%$ of its original size. They concluded that the homogeneity of the random graphs makes them resilient to cascades. A remark to be noted on their model is that it follows from the way they assign loads that on average the nodes with higher links or edges are also those with heavier load, which would lead as expected to similar conclusions as the previous static approaches to confirm the robust-yet-fragile property of the scale-free networks, as the authors indicated in their paper. The addition of this model though as compared to the static approaches is signifying the amount of damage a single node can cause to the entire system.

In this category of dynamical approaches, the work of Crucitti, Latora and Marchiori [CLM04] also proposes a model for cascading failures in complex networks, again working under the two forms of networks, scale-free and random graphs. Assume each node is given a capacity to handle traffic (or information flow, or interbank deposits in banking network), and start with a network in a stationary state where each node has a load smaller than its capacity. Now as a breakdown (removal) of node is introduced, its load has to be redistributed over other nodes. If the capacity of any of these nodes has been exceeded, this node in turn would not be able to handle the extra load and again its load has to be redistributed triggering a cascading of overloaded failures.

They consider an undirected weighted graph $G$ with $N$ nodes and $K$ edges described by its $N \mathrm{x} N$ adjacency matrix $A$ with $a_{i j}$ having a value between $(0,1]$ if there is an edge connecting node $i$ and $j$, and 0 otherwise, so that $a_{i j}$ represents the efficiency in communication between edges $i, j$. The smaller the value, the longer it takes for the two edges to communicate. The model then proceeds as follows:

1. Initial state: start at time 0 with $a_{i j}=1$ for all existing links. The load on node 
$i$, denoted by $L_{i}(0)$, is the number of shortest paths passing through the node at time 0 . The capacity of each node is then defined as the maximum load a node can have and assume it is to be proportional to its initial load

$$
\text { Capacity }_{i}=\alpha L_{i}(0), i=1,2, \ldots, N
$$

for $\alpha \leq 1$ is the tolerance parameter defined for the whole network.

2. Node removal: the removal of a node affects the efficient (shortest) paths between nodes, consequently the distribution of loads changes using the iterative rule

$$
a_{i j}(t+1)= \begin{cases}a_{i j}(0) \frac{C_{i}}{L_{i}(t)} & \text { if } L_{i}(t)>C_{i} \\ a_{i j}(0) & \text { Otherwise }\end{cases}
$$

where $j$ extends to all first neighbors of $i$. As such, if at time $t$ node $i$ is congested, we reduce the efficiency of all edges going through it.

Unlike other studies, instead of removing the nodes once overloaded, they instead reduce the efficiency on the edges going through it which eventually will force flow to take alternative paths.

Applying their model to the two types of networks, random and scale-free, using two scenarios for node removal, random and load-based, they observed a decrease in network efficiency for small values of the tolerance parameter $\alpha$ and a collapse of the system for $\alpha$ smaller than a critical value $\alpha_{c}$. Nevertheless the random network appears to be more resilient to cascading failures and in both cases the collapse transition is sharper (higher critical value $\alpha_{c}$ ) for the scenario of load-based removals.

They concluded their paper by stating that a single node breakdown can be sufficient to affect the efficiency of the network provide that node happens to be among the ones with the largest load.

Common and crucially central to both of the previous models is how the two groups of authors defined the load of the node to reflect the node centrality, that is 
the number of geodesic paths the node lies on. It should be noted that, while on average nodes with high degrees would be the ones with high centrality, this is not always the case. It can happen that a vertex with low degree also happens to be connected to others with low degree, but is a long way from others on average, and therefore still has a high centrality. This can be easily be demonstrated by vertex lying as a bridge between two groups of nodes in a network, since in such a situation any path from any node in the first groups to a node in the second group has to go through that bridge node causing it to have a high centrality [New10].

In this chapter we introduced basic notions and recent work on networks error and attack tolerance and showed how they depend on the network structure. In the next chapter we present a set of empirical studies carried on banking networks to identify their structure as well as examine their resilience. 


\section{Chapter 3}

\section{Empirical Networks}

This chapter is devoted to the introduction a set of recent empirical studies of banking networks conducted shortly before or immediately after the recent crisis (Austria 2004, Japan 2004, USA 2007, Brazil 2007, Italy 2009, and Brazil 2009). For the most part, these studies are based on actual bilateral exposures of banks, which are to be distinguished from another stream of empirical studies that relied mainly on estimation methodologies like maximum entropy or cross entropy to arrive at the interbank exposure matrix. The gap between these two streams could be as wide as concluding contradicting results.

For example, in a study carried on the Dutch interbank system by using a mixture of estimation methodologies and data sources the authors concluded through a scenario analysis of contagion risk that "none of the large bank failures trigger the failure of another large bank" [LL04]. In the same spirit, we find an elaborated study of the Belgian interbank market for the period December 1992 to December 2002 that attempts to shed light on the evolution of the network (moving from a complete network to one characterized by 'multiple money centre' structure), its stability, propagation mechanism for bank failures, and the role of a foreign banking sector [DN07]. Using an estimation mechanism for the matrix of bilateral exposures based on the assumption of banks seeking to maximize the dispersion of their interbank activities, 
they conclude that significant losses can be prevented by focussing on the resilience of large banks only, in that small banks would not cause systemic crisis as long as the large ones are resilient.

By contrast, the commonly reported finding of empirical studies based on actual exposures is that the network associated with the interbank market is scale free. As we have seen in the previous chapter, the defining feature of these kinds of networks is having a power-law distribution of the degree of vertices. In the case of banking networks, this implies that a small number of banks are "hubs" with many connections, giving rise to the notion of being "too interconnected" rather than "too big", and most other banks have few connections. In addition, another commonly reported feature in banking networks is the small-world effect, that is a small number of connections is needed to go from one bank to another, indicating that one bank's bad news is never too far from other banks.

\subsection{Pre-crisis studies}

We begin with a brief overview of a few studies conducted before the crisis, which highlight some stylized facts about empirical networks but are nevertheless not complete enough to draw comprehensive conclusions about their stability.

\subsection{Austria 2004}

Using a dataset provided by the Austrian Central Bank, Boss et la [BEST04] conducted a study on the nature of the network of the Austrian interbank market in a paper that the authors claim to be "the first to provide an empirical analysis of the structural features of a real-world interbank network using concepts from modern network theory".

The network is characterized by an exposure matrix $L$, where $L_{i j}$ are the liabilities bank $i$ has toward bank $j$. Nodes represent 900 banks in addition to the Central Bank 
(OeNB), and an aggregated foreign banking sector. Data consists of ten $L$ matrices each representing the exposure for quarterly periods between 2000 and 2003. Using two different data sources, the authors gathered about $90 \%$ of the $L$ matrices entries, and used local entropy maximization to estimate the rest.

Considering the network alternatively as a directed unweighted graph, an undirected unweighted graph, and an undirected weighted graph, they concluded that

- The degrees distribution of the interbank market follows a power law for all three types of graphs. In each of these cases there are two regions that are to be fitted by a power law (left part and tail part).

- For the undirected weighted graph, the clustering coefficient is relatively small compared to other real world networks.

- Considering the giant component of the directed graph, the network has a smallworld feature with about 3 degrees of separation.

They conclude that "both the stability of the network with respect to the random removal of nodes and the disastrous consequences of the removal of specific hubs are a direct consequence of the power laws of the degrees distribution revealed in this paper".

\subsubsection{Japan 2004}

Similarly, Inaoka et al [INST04] analyzed the network structure formed by the financial institutions in Japan using records of transactions from June 2001 executed through the BOJ-NET, an on-line system held by the Bank of Japan for funds and Japanese government securities settlements between financial institutions. As such the data used are not only for banks but also takes into account other financial institutions like security firms and credit unions.

The initial network consists of 546 financial institutions and 7,351 pairs of linked nodes. Considering the number of transactions between these pairs, the authors de- 
fined the banking network by connecting a pair of institutions when the number of transactions between the pair is greater than or equal to 21 , as they found that above the number 20 the cumulative distribution of number of transactions follows a power law. As a result the number of nodes is reduced to 354 institutions, the resulting network is for the most part composed of unconnected pairs (low density), but nevertheless the distribution of cumulative degree exhibits a power law with exponent 2.1 for degrees $k>5$.

While the paper discussed the effect of the removal of individual nodes at random on the stability of the network structure, as well as the removal of the node with the largest number of links, the authors did not actually apply that to their network. Instead they discussed the difference between two types of network structures discussed in the previous chapter, namely a random network (Erdos and Rényi) and a scalefree network (Barabási and Albert), with the underlying implicit agreement that their network is scale free.

\subsubsection{USA 2007}

Three years later, Soramäki et la published paper $\left[\mathrm{SBA}^{+} 06\right]$ exploring the nature of the interbank network in the United States using transaction data from the Fedwire Funds Service, a real-time gross settlement system operated by the Federal Reserve System where transactions are immediate and final when processed.

Considering the first quarter of 2004, and modelling each day as a separate network, the authors considered only payments between commercial banks and were motivated to examine the properties of the network before and in the immediate aftermath of the events of September 11, 2001. Compared to the previous two papers we find the authors employed a diversified set of statistical measures to characterize the network. For example they described the network components as GWCC (Giant weakly connected components), DCs (Disconnected components), and the connectivity of the network. As in the Japanese case they concluded that the network is 
extremely sparse with $99.7 \%$ of the potential links not used. In addition they found that the number of links and nodes are both correlated with the value and volume of payments settled. Similar to the above two studies, they found a small-world feature with an average path length of $2.6 \pm 0.02$.

As for the degree distribution, they report a power-law tail of 2.11 for degrees $k>10$. On the other hand, and unlike the Austrian network, the authors reported a high clustering coefficient (after ignoring nodes with a degree smaller than three). In addition, they found the network to be disassortative, that is, high degree nodes are attached to low degree nodes.

\subsubsection{Brazil 2007}

In the same year, a paper by Cajueiro and Tabak [CT07] applies a weighted directed network approach to the interbank information provided by financial institutions to the Central Bank of Brazil. The authors collected data from 86 banks and 23 nonbank financial institutions for the period from January 2004 to December 2005, with a main interest on the role of different banks to the interbank market, where they classify banks according to size, ownership and type.

They report a structure compatible with scale-free networks. Nevertheless, no further information was provided on the nature of the distribution nor its exponent. In addition to using the in-degrees and out-degrees (weighted and unweighted), where they found a strong positive correlation between in-measures and out-measures, they

used a minimal spanning tree measure to conclude the existence of communities in the interbank markets (private, foreign and public banks are connected among themselves). 


\subsection{Post-crisis studies}

While all the papers mentioned above documented a scale-free network, none examine in detail the consequences of individual node failure (whether random or intentional) on the resulting network. Following the crisis, we find a number of empirical studies that attempt to introduce measures of resilience and examine the stability of the network under different scenarios of shocks.

\subsubsection{Italy 2009}

This study by Lazzetta and Manna [LM09] used a dataset of 222 monthly data points starting from January 1990 to June 2008 of month-end gross unconsolidated bilateral interbank deposits reported by the Italian banks to the Central Bank of Italy, with a sample of 1146 banks at the beginning of the study period and ending with 790 banks.

One addition the paper provided to the above empirical studies is a historical view on the network and how it had changed over time, for example they found that the amount of local interbank deposits as a percentage of total assets of the interbank system grew considerably and that the interbank market also become more concentrated, measured as the market share of Italy's largest 50 banks.

Similar to the above Brazilian network they found a strong positive correlation between in and out-degrees. On the other hand, similar to the USA study, they report the network to be disassortative. A scale-free distribution is once again observed, coupled with a small-world feature of average path length around 2.5. It is worth mentioning that over the examined period the average path length has increased from 2.1 to 2.4 .

Regarding measures of resilience, they started by examining the effect of removing a single bank at a time and identifying which of them would render the network disconnected. They reported that the number of banks with this property had increased 
from 5 or less at the beginning of the examining period to around 15 by the end of the examining period. Building on that finding the authors computed two measures of resilience. The first consists of identifying the set of these banks that will make the network disconnected. They then simulate their removal and measure the area of the network which becomes cut from the core. They concluded that "while global hubs have increased in role (when assessed against their potential for contagion), local hubs have increased in number". Accordingly, the rise of local hubs indicates that guaranteeing the smooth operation of the big banks, identified as major market players, is no longer a sufficient condition for the smooth circulation of funds across the whole network.

The second measure depends on identifying the banks with highest geodesic frequency. An important remark they made is that the group of banks whose removal may render the network disconnected does not overlap with the banks with the high geodesic frequency, or with the group of banks running large volumes of interbank deposits.

\subsubsection{Brazil 2009}

The more recent study of Cont and Santos [CS09] takes pride in building the analysis on a complete set of detailed real data for an interbank market. They refer to the study as being the first in that regard. In addition, the study aimed at exploring the relation between banks' capital buffers and the connectivity of the interbank network through two resilience measures: the default impact, where the contagion impact of single bank default on the network is measured, and the systemic risk index, where they consider not only contagion but also the effect of systemic events on the network.

Their database consists of 6 dates (June 2007, December 2007, March 2008, June 2008, September 2008 and November 2008). They used interbank exposure for all financial institutions contemplating all sources of risk (fixed income instruments, borrowing and lending, derivatives, and foreign exchange). They presented the interbank 
market as a directed weighted network $\Gamma_{t}=\left(V_{t}, L_{t}, C_{t}\right)$ for $t=1,2, \ldots, T$, where $V_{t} \in\left\{1,2,3, ., n_{t}\right\}$ is the number of vertices at time $t, L_{t}=\left(l_{i j}\right)_{t}$ is the $n_{t} \times n_{t}$ matrix of exposures of bank $i$ to bank $j$ at time $t$, and $C_{t}=\left(C_{r, i}, C_{1, i}, C_{2, i}\right)_{t}$ is the capitalization structure of each bank according to the following three types of capital:

- Required Capital as a function of the associated risks of each financial intuitions operations computed as

$$
C_{r, i}=\alpha \times \text { Risk Base, }
$$

where $\alpha$ is the minimum required Basel Index and equals to 0.11 for the Brazilian case, while the risk base is the sum of different risk components (credit exposures weighted by their respective risk, foreign currencies and gold exposures; interest rate exposures; commodity prices exposures; stock prices exposures and operational risk exposures).

- Tier 1 Capital $C_{1, i}$.

- Reference Capital $C_{2, i}$ given as the sum of tier 1 Capital and tier 2 Capital.

Denoting by $A_{t}=\left(a_{i j}\right)_{t}$ the adjacency matrix associated with $L_{t}$, the in-degree for a vertex $i \in V_{t}$ in the network is defined as

$$
k_{i n, i}=\sum_{j \in V_{i n, i}} a_{i j}
$$

the out-degree as

$$
k_{\text {out }, i}=\sum_{j \in V_{\text {out }, i}} a_{j i}
$$

and total degree as $k_{i}=k_{\text {out }, i}+k_{\text {in }, i}$, where $V_{\text {in }, i}=\left\{j: a_{i j}=1\right\}$ and $V_{\text {out }, i}=\left\{j: a_{j i}=1\right\}$. Similarly, the in-weighted degree, out-weighted degree and total weighted degree for 
financial institution $i$ are defined as:

$$
\begin{aligned}
w_{\text {in }, i} & =\sum_{j \in V_{\text {in }, i}} l_{i j} \\
w_{\text {out }, i} & =\sum_{j \in V_{\text {out }, i}} l_{j i} \\
w_{i} & =w_{\text {out }, i}+w_{\text {in }, i}
\end{aligned}
$$

The authors find support for the statistical significance of the following hypotheses about the empirical network:

1. Stationarity of degrees: The in-degree, out-degree and degree distributions are stationary.

2. Power-law distribution of degrees: Above a certain threshold $k_{m i n}$, the outdegree, in-degree and degree distributions follow a discrete power law with exponent ranging from 2 to 3 .

3. Power law distribution of the exposure size: Above certain threshold the distribution for exposures follows a continuous power law.

4. Relation between exposure size and connectivity: The number of degrees $k_{i}$ and mean weighted degree $w_{i} / k_{i}$ for financial institution $i$ are not independently distributed (while the authors could not confirm the existence of linear relationship, they cannot reject the hypothesis that there is a nonlinear relationship).

5. Capital buffer linear model: the best linear model describing the capital buffer $C_{e 2}:=C_{2}-C_{r}$ in terms of in-degrees, out-degrees, weighted in-degrees, weighted out-degrees, weighted degrees, and degrees is

$$
C_{e 2, i}=\beta_{0}+\beta_{1} \times w_{i n, i}+\epsilon_{i}
$$

where the least-squares estimates for the coefficient are $\beta_{0}=50.8826$ and $\beta_{1}=$ 
0.1887. It is worth noting that the $R^{2}$ (coefficient of determination) value for all the linear models they examined are below 0.5 and for that particular "best model" the value is $R^{2}=0.4370$. In that regard, they attributed the remainder of capital buffer in the linear model to be the part of resources allocated to non-interbanking operations.

6. Distribution of residuals: The residuals $\epsilon_{i}$ in the linear model above follow a scaled $t$-distribution.

Building on their previous definition for capital buffer $C_{e 2}$, they introduced a definition of capital buffer that is devoted to absorb losses only related to banking exposures that is

$$
\bar{C}_{e 2, i}=C_{2}-C_{r, i}+\alpha \times \sum_{j \in V_{i n, i}} l_{i j}
$$

Using the new capital buffer they introduced the definition of default of a financial institution if its modified capital buffer is negative, that is, the condition of default for institution $i$ is $\bar{C}_{e 2, i}<0$.

Upon the default of individual bank, the risk propagates through a write-off procedure. At time $t$, if bank $i$ has a reason to suspect that counter-party $j$ will not honour its obligations $l_{i j}$, then bank $i$ will write off that exposure from its asset portfolio and consequently also reducing its capital buffer by the same amount. If that causes bank $i$ to default as well, then a series of write-off procedures might take place triggering default via contagion.

Specifically, let the set $D^{(s)}=\left\{j \in V_{t}: \bar{C}_{e 2, j}^{(s)}<0\right\}$ represents the financial conglomerates in default in state s, where $\bar{C}_{e 2, j}^{(s)}$ for all $j \in V_{t}$ are the capital buffers in this state $s$. They modeled the process of contagion via the states notations, where they started their model at state 0 . Then the write-off procedure will be given by the buffer dynamics

$$
\bar{C}_{e 2, i}^{(s+1)}=\bar{C}_{e 2, i}^{(s)}-\sum_{j \in D^{(s)}} l_{i j}
$$


where $s+1$ is the subsequent state. As stated, the write-off procedure may cause further defaults and continues until the financial system finds an equilibrium state $s^{*}$ where no more defaults are being caused:

$$
s^{*}=\inf \left\{s: D_{0}^{(s)} \backslash D_{0}^{(s+1)}=\phi\right\}
$$

The default impact of a given bank default can then be measured as the total percentage of total capitalization lost in the system. As such, it can be defined as

$$
D I_{j}=\frac{\sum_{i \in V_{t}}\left\{\max \left(\bar{C}_{e 2, i}^{(0)}, 0\right)-\max \left(\bar{C}_{e 2, i}^{\left(s^{*}\right)}, 0\right)\right\}}{\sum_{i \in V_{t}}\left\{\bar{C}_{e 2, i}\right\}}
$$

given that the initial defaulting set is $D_{0}=\{j\}$. Applying their definition to the network, the authors reported an exponential shape for the histogram of the observed $D I$, with most financial institutions not destroying more than $4 \%$ of the total capitalization of the system, and with few institutions that could destroy as much as $15 \%$.

In addition to default impact, the authors introduce a systemic risk index to account for the systemic events case. The idea follows from hitting the system with an exogenous shocks (such as market information or credit information) that could affect the capital buffer of all institutions simultaneously. The systemic risk index of financial conglomerate $i \in V_{t}$ is given by

$$
S I_{i}=E\left[D I_{i}^{\epsilon} \mid \bar{C}_{e 2, i}+\sigma_{i} \epsilon_{i}<0\right]
$$

where the default impact $D I_{i}^{\epsilon}$ is computed considering the capital buffer after the effects of exogenous shock $\epsilon_{i}$, and $\sigma_{i}$ is a scale factor to adjust the exogenous shocks for the credit risk.

In simulating the shock, the authors started with a sequence of correlated uniform random variables which in turn is used to generate the sequence of heavy tail random 
variables $\epsilon_{1}, \epsilon_{2}, \ldots ., \epsilon_{n}$ through the inverse cumulative density function of the scaled t-distribution (see hypothesis 6 above). On the other hand they estimated $\sigma_{i}$ using the historical probability of defaults $\pi_{i}$ for the financial institutions..

Using Monte Carlo simulation to calculate the expectation in (3.2), the authors reported a lognormal shape for the histogram of the $S I$ distribution, indicating similarly a very few institutions that can destroy up to $40 \%$ of the system capitalization. Finally the authors concluded their paper by discussing the liquidity effect. They incorporated liquidity risk in times of hardship by modifying the capital buffer to

$$
\bar{C}_{e 1, i}=C_{1}-C_{r, i}+\alpha \times \sum_{j \in V_{i n, i}} l_{i j}
$$

That is, they replace the reference capital (which is tier $1+$ tier 2 capital) by only tier 1 capital. The results in terms of the shape of SI and DI distribution hold as before.

If we are to compare the study of the Italian network in [LM09] to this more recent one of Cont and Santos [CS09] considering the Brazilian case, we find that while the former shed more light on the historical trends and development of the network, the Cont and Santos study is directed more toward a quantitative detailed description of the network with a heavy employment of statistical significance analysis to their findings. As such we take the Cont and Santos study as our starting point for examining the contagion risk on a simulated network of the interbank market. In the next chapter, we build a simulated network resembling the characteristics documented in the empirical studies presented above, and examine the simulated network resilience. 


\section{Chapter 4}

\section{Simulated networks}

As presented in the previous chapter, the structure of typical empirical interbank market resembles that of a scale-free network, which in turn has a clear implication on the resilience of the network in face of shocks and cascading failures (see section 2.3.2). In this chapter we study the resilience of the interbank network from a simulation point of view. We start by building a simulated network mimicking the characteristics documented in the empirical studies. We then follow Cont and Santos [CS09] and shock the network with bank failures and systemic events and study their effects in terms of losses in the interbank system capitalization. We also examine the number of banks led to default following an initial bank failure and demonstrate the role played by the most connected banks in our system.

\subsection{Network construction}

Combining the empirical findings reviewed in Chapter 3 with the network formation mechanisms discussed in Chapter 2, it follows that what we are looking for is a mechanism that generates a power-law degree distribution for a directed weighted network. As such, our model would ideally rely on a preferential attachment mechanism that extends the Barabási and Albert model ([AB00], [AB02]) to the case of a weighted 
directed network. In addition we should use a model that allows the possibility of obtaining different in-degree and out-degree distributions in terms of different exponents for their power-law distributions. To start, we focus on generating an unweighted directed scale-free network for bank links, then consider populating the network with the sizes of exposures later.

\subsubsection{Preferential attachment in a directed unweighted net- work}

We adopt the mechanism introduced by Bollobas et al in [BBCR03]. As in Barabási and Albert [BA99], we start with an initial fixed directed graph $G_{0}$ with $t_{0}$ edges (for example a single vertex with no edges). Let $\alpha, \beta, \gamma, \delta_{\text {in }}$, and $\delta_{\text {out }}$, be non-negative real numbers such that $\alpha+\beta+\gamma=1$. Vertices are then added gradually at each time-step as follows:

1. With probability $\alpha$, add a new vertex $v$, together with a directed edge, originating from the new vertex $v$ to an existing vertex $w_{i}$ chosen at random with probability proportional to $k^{i n}(w)+\delta_{i n}$, that is, proportional to the in-degree of vertex $w$ plus a constant. In other words, for a graph $G(t)$ with exactly $t \geq t_{0}$ edges and $n_{t}$ vertices, the probability of choosing vertex $w$ is

$$
p\left(w=w_{i}\right)=\frac{k^{i n}\left(w_{i}\right)+\delta_{i n}}{\sum_{i=1}^{n_{t}} k^{i n}\left(w_{i}\right)+\delta_{i n}}=\frac{k^{i n}\left(w_{i}\right)+\delta_{i n}}{t+\delta_{i n} n_{t}} .
$$

2. With probability $\gamma$, add a new vertex $v$ together with a directed edge from an existing vertex $w$ chosen with probability proportional to $k^{\text {out }}(w)+\delta_{\text {out }}$ to the new vertex $v$.

3. With probability $\beta$, add an edge from an existing vertex $v$, chosen with probability proportional to $k^{\text {out }(v)}+\delta_{\text {out }}$, to another existing vertex $w$, chosen with a probability proportional to $k^{i n}(w)+\delta_{i n}$. 
It should be noted from the algorithm above that the preferential attachment criteria is not purely proportional to the in-degree or out-degree of vertices, rather it is proportional to them plus positive constants $\left(\delta_{\text {in }}\right.$ and $\left.\delta_{\text {out }}\right)$. This is because using a purely proportional mechanism would mean that new vertices with zero edges (in-edge) will have a zero probability of getting new edges (in-edges), and similarly for out-edges, thereby producing vertices with either in-edges or out-edges but not both. It should be also noted that this model does not prevent loops or multiple edges, however these should be negligible in large networks.

The authors proved that under the conditions of $\alpha \delta_{\text {in }}+\gamma>0$ and $\gamma \delta_{\text {out }}+\alpha>0$, in the limit the above procedure produces a scale-free network with in-degree following a power-law distribution with exponent $X_{I N}$ and out-degree following a power-law distribution with exponent $X_{O U T}$ given by:

$$
\begin{aligned}
& X_{I N}=1+\frac{1+\delta_{\text {in }}(\alpha+\gamma)}{\alpha+\beta} \\
& X_{\text {OUT }}=1+\frac{1+\delta_{\text {out }}(\alpha+\gamma)}{\gamma+\beta}
\end{aligned}
$$

As reported in the empirical analysis carried by Cont and Santos [CS09], the Brazilian network in March 2008, consists of 133 institutions and exhibits in-degree power-law distribution with exponent 2.2059 and out-degree power-law distribution with exponent 3.4. Note that these are tail power law distributions with the cut-off at $k_{\min }=7$ and $k_{\min }=16$ respectively, nevertheless we shall be ignoring the cut off for now. Replicating their results using the algorithm above requires a proper choice for the parameters $\beta, \gamma, \alpha, \delta_{\text {in }}$, and $\delta_{\text {out }}$. For simplicity, in case of adding a new vertex we assume that the probability of having in-edge is equal to the probability of out-edge, that is $\alpha=\gamma$. In addition, we use the equations for $X_{I N}$ and $X_{O U T}$ above to determine the constants

$$
\delta_{i n}=\frac{\left(X_{I N}-1\right)(1-\alpha)-1}{2 \alpha}
$$




$$
\delta_{\text {out }}=\frac{\left(X_{\text {OUT }}-1\right)(1-\alpha)-1}{2 \alpha}
$$

Hence, the structure depends on the choice of the probability $\alpha$ (or equivalently $\beta$ ), which should be chosen in such a way to ensure that $\delta_{\text {in }}$ and $\delta_{\text {out }}$ are positive. In what follows, we choose the values reported in Table 4.1, resulting in the networks shown in Figures 4.1 to 4.3 .

Table 4.1: Parameters values for choice of attachment probability $\alpha$

\begin{tabular}{ccc}
\hline \hline$\alpha$ & $\delta_{\text {in }}$ & $\delta_{\text {out }}$ \\
\hline 0.05 & 1.46 & 12.8 \\
0.1 & 0.43 & 5.8 \\
0.15 & 0.08 & 3.47 \\
\hline
\end{tabular}

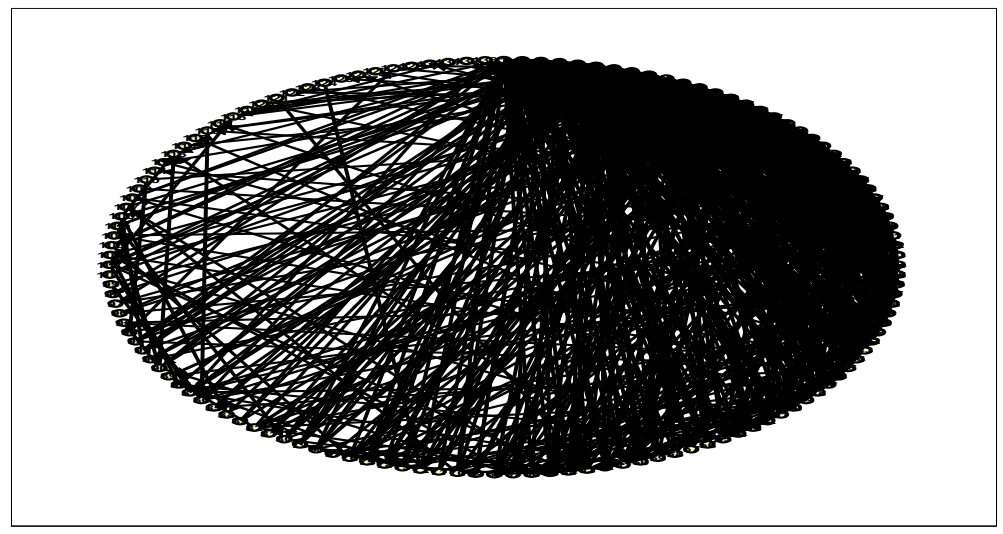

Figure 4.1: Network for $\alpha=0.05$ 


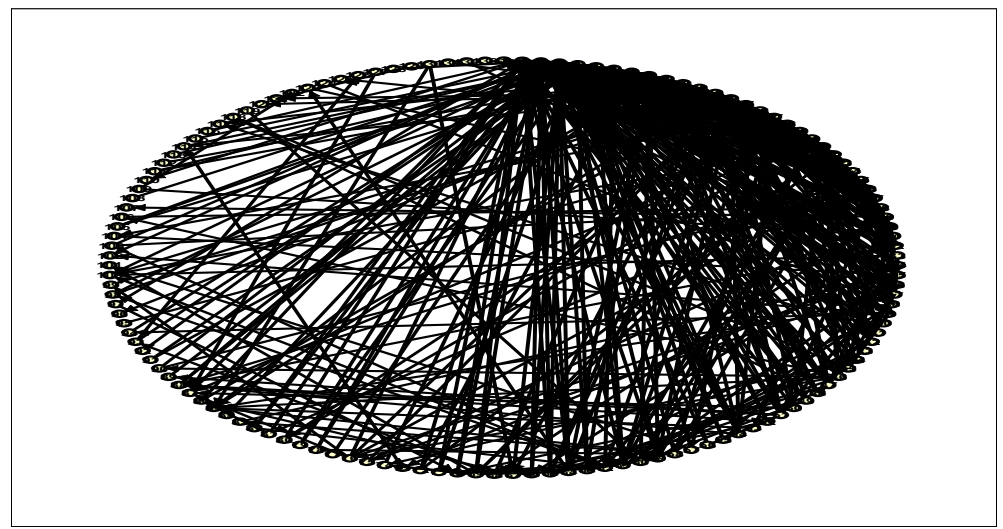

Figure 4.2: Network for $\alpha=0.1$ 


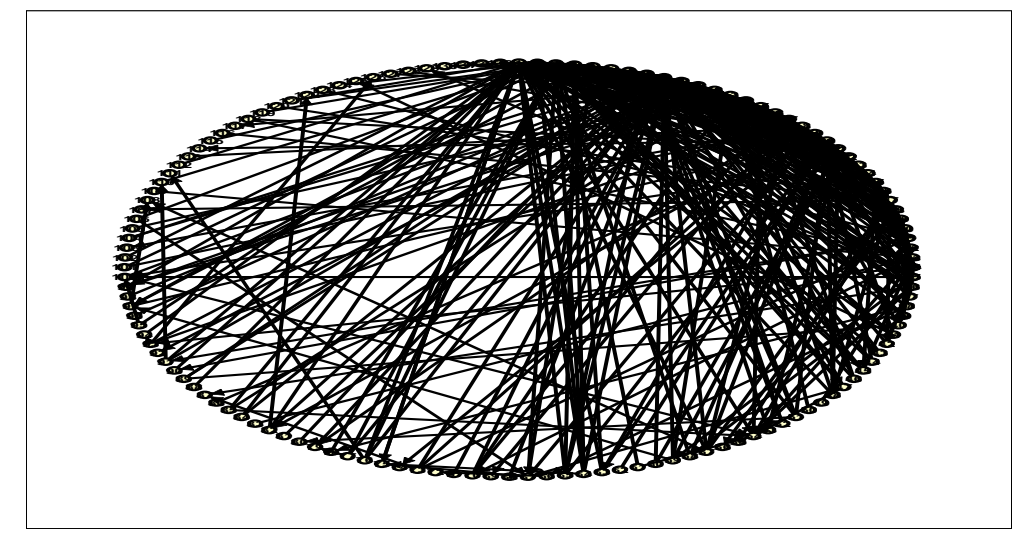

Figure 4.3: Network for $\alpha=0.15$

The plotted graphs start at the top centre (noon hour), with banks and links being added clockwise using the mechanism described above. The procedure stops when the number of desired banks (in our case 133 banks) has been reached. Upon visual inspection, it is obvious that moving clockwise, the network becomes less dense and the number of links clearly decreases, reflecting the anticipated effect of a scalefree distribution. Another observation is that for the low value of $\alpha=0.05$, the network seems much denser than the other two networks.

To verify our initial observations, we draw the in-degree, and out-degree distribution for the three networks in Figure 4.4. As seen from the histograms, the case of low $\alpha$ value produces a more dispersed distribution with the existence of banks with high in-degree/out-degree (hubs) compared to the other two cases of higher attachment probabilities $(\alpha=0.1$ and $\alpha=0.15)$. This is because for the case of low probability of adding new vertices $(\alpha=0.05)$, existing vertices have a higher chance of getting connected to each other. 

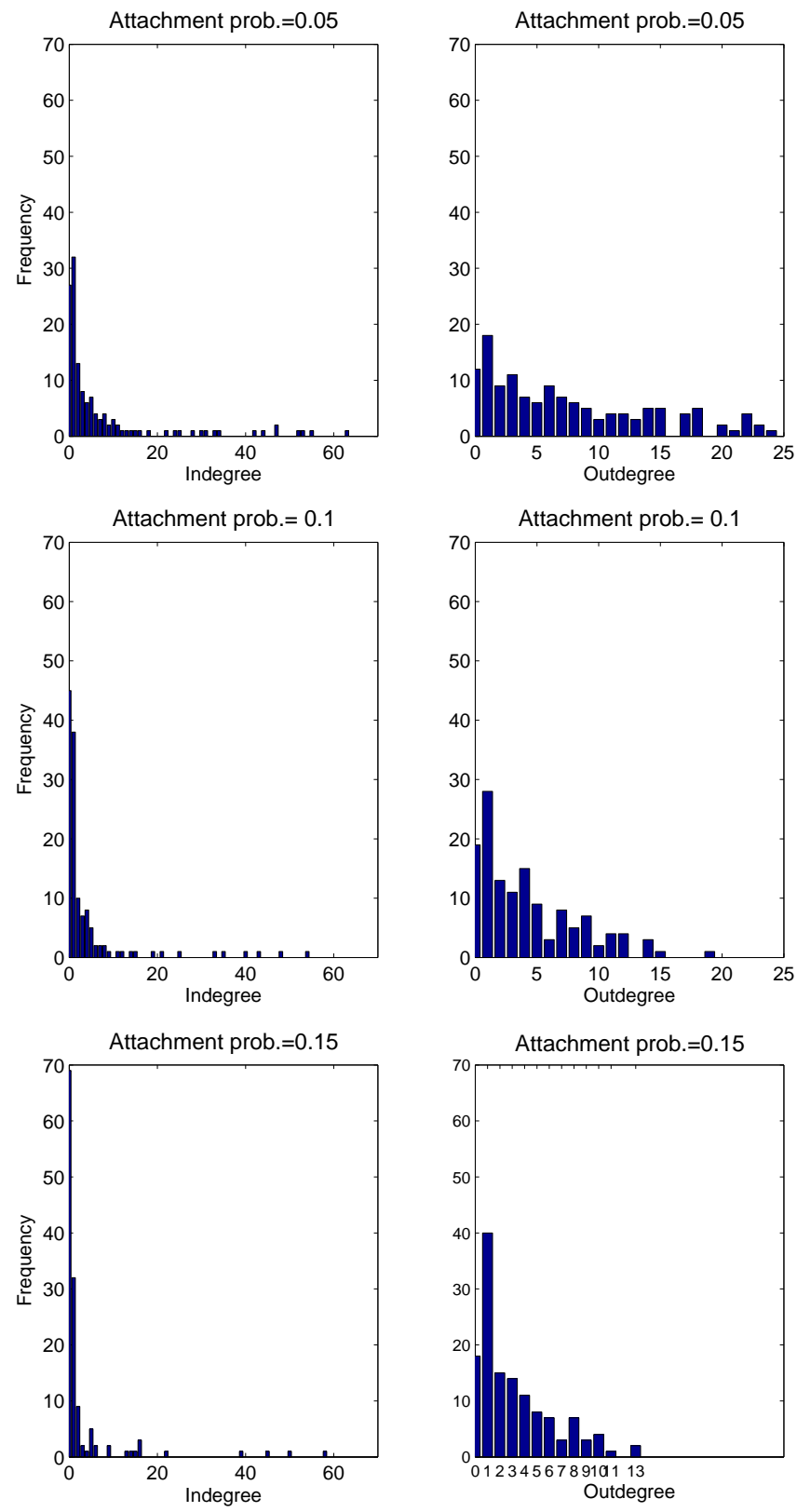

Figure 4.4: Histograms for the in and out-degree distributions for different $\alpha$ (attachment probabilities) values.

\subsubsection{Examining the simulated network}

Before moving to the second step of populating our network, it is worth pausing to examine the kind of network generated by the algorithm above. The method we 
used for generating the scale-free directed network following the work of Bollobás et al produces results theoretically holding in the limit. While they applied their method to generate an actual network with the observed in-degree and out-degree distributions, they simulated the worldwide web graph, that is a network with more than 20 million pages (vertices) and about 1.5 billion links. As such, we need an intermediate step of verifying the validity of the simulated network particularly since we are dealing with less than a few hundred nodes.

Verifying the validity of the simulated network can be achieved in one of two ways:

- assessing the goodness of fit of the simulated network in relation to the hypothetical power-law distribution with the desired exponents;

- estimating the empirical distribution of the simulated network.

We believe the second approach to be the more appropriate one, particularly when considering the divergence between simulated and actual exponents given in the example of Bollobás et al for the worldwide web graph [BBCR03]. We follow Clauset, Shalizi and Newman[CSN09], where they presented a framework for quantifying the power-law behaviour in multiple empirical data sets.

Let us start by assuming that the method we followed generated a network exhibiting power law in-degree and out-degree distributions. Our task is then to find the parameters of the distributions that best fit the data. Power-law distributions as previously indicated possesses two parameters: the exponent parameter or scaling, and the cut-off value, which we have not dealt with so far. If we assume for now that the cut-off $k_{\min }$ parameter value is known, the MLE of the exponent of the power law distribution is

$$
X=1+n\left[\sum_{i=1}^{n} \ln \frac{k_{i}}{k_{\min }}\right]^{-1}
$$

where $k_{i}, i=1, \ldots n$ are the observed values of $k$ such that $k_{i} \geq k_{\text {min }}$ which can be easily calculated ${ }^{1}$.

\footnotetext{
${ }^{1}$ We follow the common practice of assuming our data to follow a continuous power-law distri-
} 
In estimating the cut-off on the power-law distribution, we follow their method of choosing that cut-off value which makes the probability distribution of the measured data and the fitted data (with the exponent estimated above), as close as possiblevabove that cut-off value $k_{\text {min }}$. As such we proceed as follows

1. propose a cut-off $k_{\min }$;

2. find the MLE for the exponent for that cut-off value;

3. for values above the cut-off, measure the distance between the simulated distribution and the estimated one using the Kolmogorov-Smirnov ${ }^{2}$ (KS) statistic

$$
D=\max _{k \geq k_{\min }}|S(k)-P(k)|,
$$

where $S(k)$ is the CDF (cumulative distribution function) of the data of observations with a value at least $k_{\text {min }}$, and $P(k)$ is the best fit power-law model for the data in region $k \geq k_{\text {min }}$.

4. estimate $k_{\min }$ which minimizes $D$ (through comparing the different $D$ values for each of the proposed cutt-offs).

Before applying our approach to the networks simulating the Brazilian banking system for March 2008, it is worth noting that for such a small sample sizes of around one hundred nodes it becomes hard to distinguish between data drawn from the closely related power-law, log normal and exponential distributions, particularly as our fit is also controlled by the cut-off value. As stated in [CSN09], for samples of size of about a hundred "we can not accurately distinguish the data sets because there is simply not enough data to go on".

The results for our simulated networks are reported in Table 4.2. For each of the three networks we report the estimated parameters, the KS statistics and the KS bution to simplify our calculations.

${ }^{2}$ For non-normal data, the Kolmogorov-Smirnov is the commonest used measure for quantifying the distance between two probability distributions [CSN09]. 
tabulated value ${ }^{3}$. In all cases we see that the KS statistics is less than KS tabulated values, so we cannot reject the hypothesis that our data does actually follow a powerlaw distribution with the reported parameters. Finally in the last row entry of our table we report the number of data points above the cut-off value, that is the number of banks having degrees greater than $k_{\text {min }}$.

As obvious from the fitted values in the table, the case for network with $\alpha=0.15$ performs the worst, under a $1 \%$ level of significance. It produces a very high cut-off parameter for the out-degree/in-degree distribution, as only 20 data points in our network is above that cut-off for the out-degree, and 23 for the in-degree.

\begin{tabular}{|l||l|l|l|l|l|l|}
\hline \multicolumn{1}{|c||}{} & \multicolumn{2}{l|}{$\alpha=0.15$} & \multicolumn{2}{l|}{$\alpha=0.1$} & \multicolumn{2}{l|}{$\alpha=0.05$} \\
\cline { 2 - 7 } & In-deg & Out-deg & In-deg & Out-deg & In-deg & Out-deg \\
\hline \hline Cut off $\left(k_{\min }\right)$ & 3 & 7 & 3 & 3 & 6 & 5 \\
Exponent & 1.7903 & 5.1620 & 2.0386 & 2.4150 & 2.0055 & 2.3092 \\
KS statistic & 0.1209 & 0.1500 & 0.1750 & 0.1507 & 0.1042 & 0.1283 \\
KS tabulated & 0.30728 & 0.32688 & 0.2577 & 0.1908 & 0.2577 & 0.1870 \\
Data $>$ cut off & 23 & 20 & 40 & 73 & 40 & 76 \\
\hline
\end{tabular}

Table 4.2: Parameters estimated for power law fitting of the simulated networks.

\subsubsection{Directed weighted network}

Having obtained a satisfactory directed scale-free network with the desired degree distributions, the next step is to populate the network with exposures. The populating method depends crucially on the underlying assumption on the relationship between the node connectivity (node degrees) and the amount of exposure it bares (mean weighted degrees). As stated previously, Cont and Santos [CS09] rejected the hypothesis of the existence of a linear relationship between the two variables in their examination of the Brazilian network, but nevertheless could not reject that of a nonlinear relationship (Hypothesis 4). For simplicity we assume that the two variables, degrees and weights, are independent. As such the weights can be modelled as identi-

\footnotetext{
${ }^{3} \mathrm{KS}$ tabulated values reflect $1 \%$ level of significance
} 
cally distributed random variables drawn from a suitable distribution, and populated into our built unweighted directed network (i.e. populate it into the adjacency matrix). In determining the suitable distribution, we count again on the assumption of a continuous power-law distribution, following the findings of Cont and Santos (Hypothesis 3).

\subsection{Stability}

Having built the simulated directed weighted network, in this section we build bank buffers and examine the resilience of the simulated network as banks are subjected to shocks as well as systemic events

Following Cont and Santos [CS09], we first build bank buffers as indicated earlier (Hypothesis 5, 6):

$$
\bar{C}_{e 2, i}=\beta_{0}+\beta_{1} \times w_{i n, i}+\epsilon_{i}+\alpha \times \sum_{j \in V_{i n, i}} l_{i j}
$$

where the coefficients are $\beta_{0}=50.8826$ and $\beta_{1}=0.1887, \alpha=0.11$ is the minimal required Basel Index, and the residuals $\epsilon_{i}$ follows a scaled $t$-distribution.

The initial analysis for default mechanism is through examining single-bank defaults expressed by the default impact measure $D I$ defined in equation (3.1). As such, we proceed by hitting one bank at a time and measure the impact on the network. We analyze the effect of the network structure on the systemic risk by considering both a scale-free network and a random network. It is worth noting that while we expect to witness differences between them, our conclusions are limited by our small network size. Thus, we enlarge the size of the networks to examine the impact of the two different network topologies. Next we focus on the role of "too interconnected" banks in the scale-free network. Finally we conclude our analysis by assessing the performance of the network under the systemic events case as reflected in the systemic risk index (defined in equation (3.2)). 


\subsubsection{Single bank defaults in scale-free banking networks}

We start by constructing a weighted directed network with $\alpha=0.05$, based on the directed network built earlier ${ }^{4}$. We then examine the $D I$ impact, or the percentage of capital lost ${ }^{5}$ in the network caused by a single-bank defaults. We find that most of the banks will cause a loss of less than $4 \%$ while only one bank causes a loss of as large as $12 \%$.

A similar conclusion has been reached in [CS09], as they reported that most banks will cause an impact of not more than $4 \%$, nevertheless few will cause an impact of as big as $15 \%$.

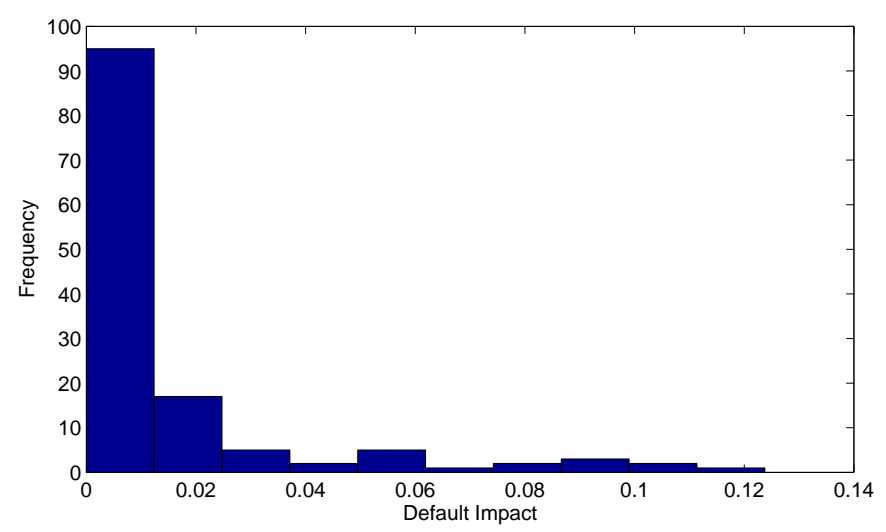

Figure 4.5: Histogram of $D I$ caused by a single bank default in a scale-free network.

Another interesting question is how losses relate to the defaulted bank own size. That is the ratio of total capitalization lost in the system to the bank's own capitalization. As shown in the histogram in Figure (4.6), most of the banks will cause losses as high as five times their sizes, nevertheless we find that few banks causes losses that can reach up to 20 times their size.

\footnotetext{
${ }^{4}$ Using the parameter values used earlier, combined with parameter values from [CS09] to replicate the observed network for Brazilian system in March 2008.

${ }^{5}$ Excluding the defaulted bank capitalization.
} 


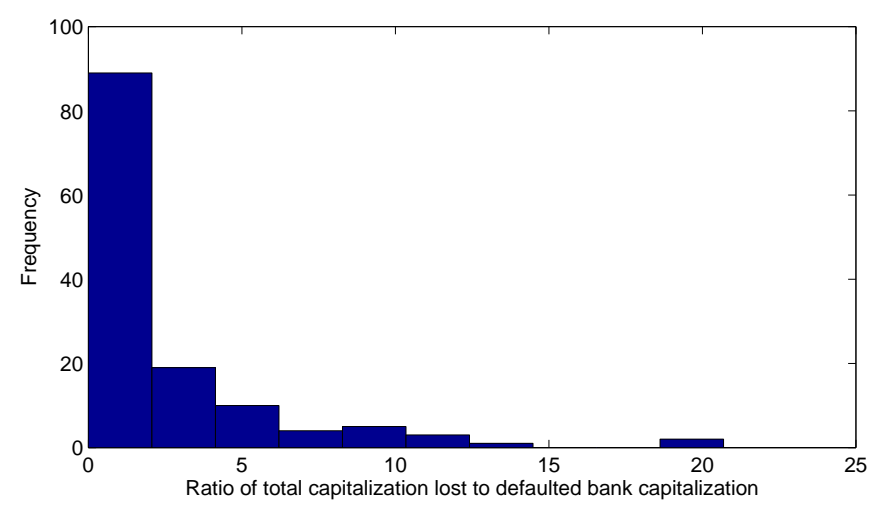

Figure 4.6: Histogram of the ratio of the total capitalization lost to the defaulted bank capitalization in a scale-free network.

While lost capitalization as measured by $D I$ should capture losses in the system, an additional dimension is how many other banks have been pushed to default as a consequence of the originally defaulted bank. As indicated in some recent studies, the notion of "too interconnected to fail" has been gaining wide support since the crisis. The statement can be examined both by the $D I$ impact and by the number of defaults that highly connected banks would cause.

For the second criterion, upon examining the relation between number of indegrees the defaulted banks have and the number of additional banks the initial default triggered, we find that while most banks will not trigger further defaults, a few banks will trigger multiple defaults (as high as causing another 4 banks to default). These high risk banks, as shown in Figure 4.7, have high in-degree, nevertheless they are not necessarily the most interconnected banks. 


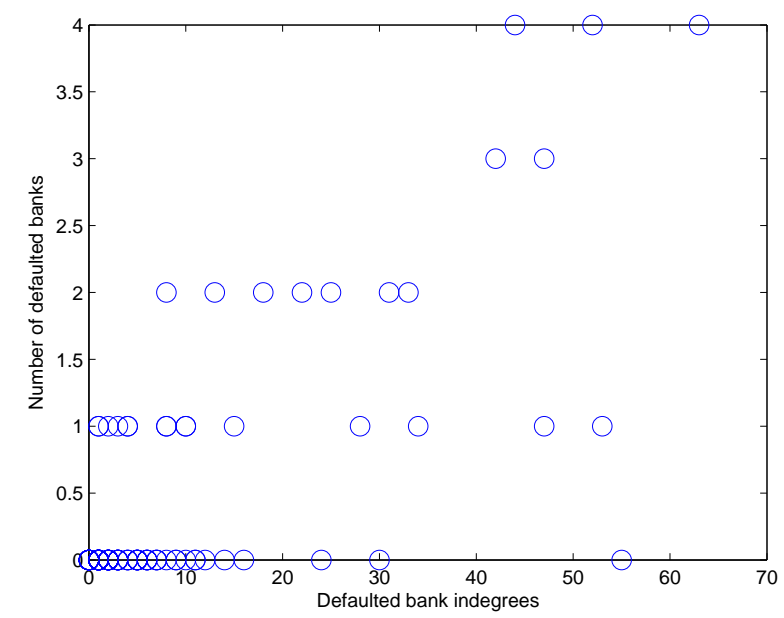

Figure 4.7: Relation between number of the defaulted bank in-degrees and the number of subsequent defaults in a scale-free network.

The relation between "too interconnected" and the system fragility is more apparent in terms of default impact $D I$. As we see in Figure 4.8, there is a clear positive relation between the two variables, with the most interconnected bank causing the highest percentage loss in the system total capitalization

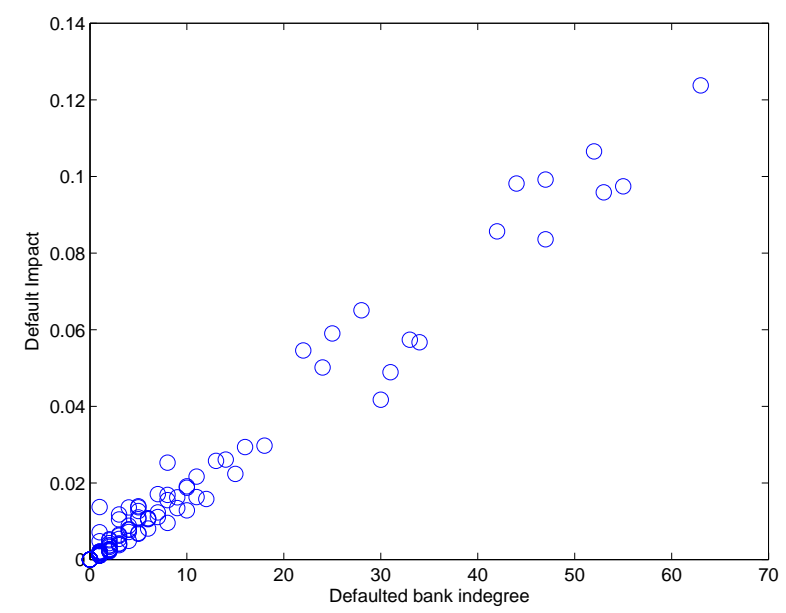

Figure 4.8: Relation between in-degrees of defaulted banks and the percentage of capitalization lost in a scale-free network. 


\subsubsection{Single bank defaults in large networks}

As mentioned earlier, the considerably small network in the example of the Brazilian banking system does not allow us to draw firm conclusions in terms of the impact of network structure on its stability. As such, by enlarging our network to 500 nodes (producing 4,279 links in the scale-free network), and keeping our parameter values as before $\left(\alpha=0.05, \delta_{\text {in }}=1.46, \delta_{\text {out }}=12.8, \beta_{0}=50.8826, \beta_{1}=0.1887\right)$, a clearer differentiation between the effect of structure can be drawn. We also built a random network $G(n, m)$ with $n=500$ nodes and edges $m=4,279$ and populate both networks using a Pareto distribution for exposures.

For the scale-free network we find banks with in-degrees as high as almost 165 (hubs) compared to less than 20 in-degree in the random network case, as shown in the histogram in Figure 6.4. In the same histograms, we see the clear difference in the distribution of the in-degrees for banks in the two networks, with a clear long tail distribution for the scale-free network. 

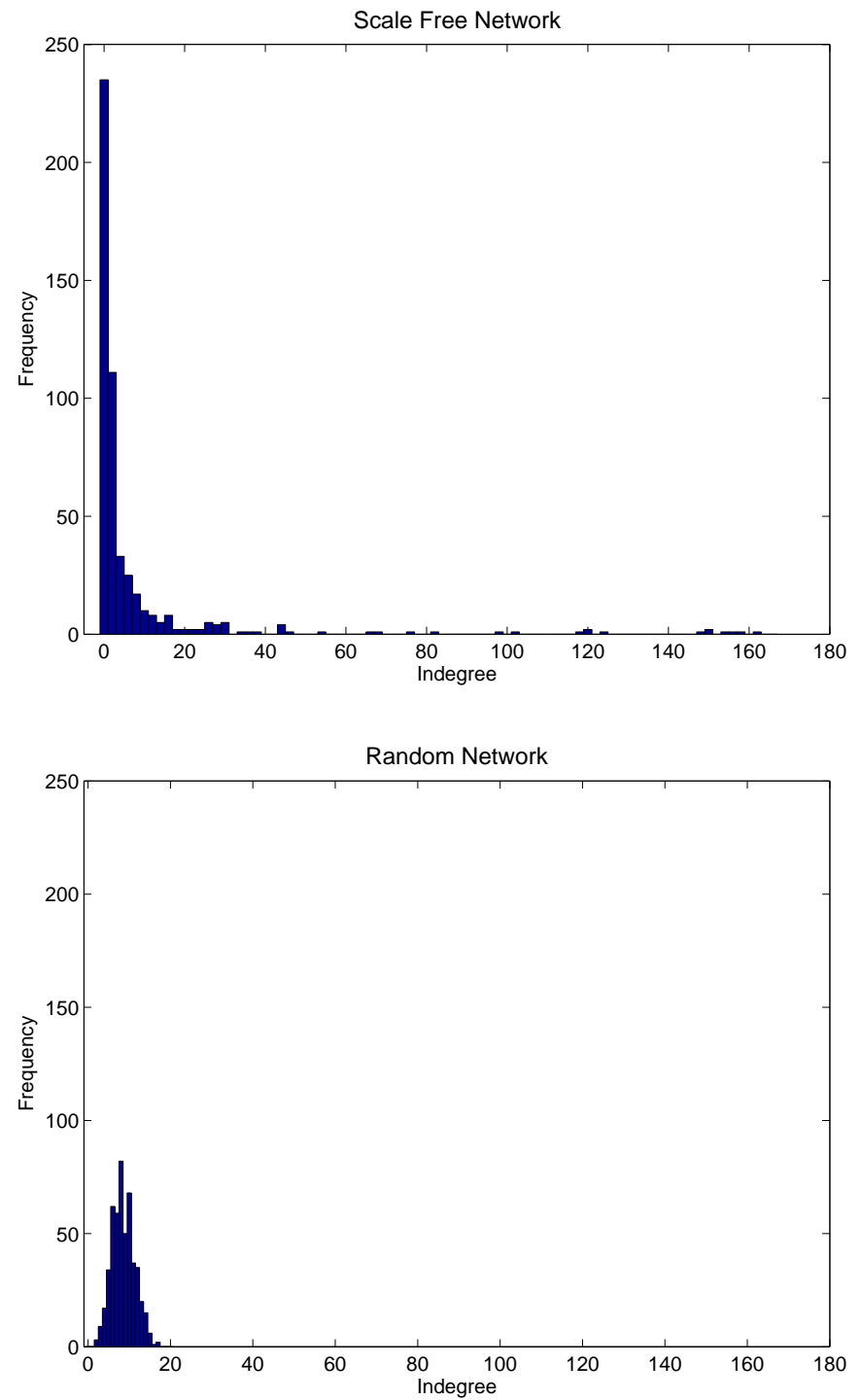

Figure 4.9: Histogram of banks in-degree for scale-free and random networks.

For the histogram of default impact, it is obvious that the distribution of total losses in the two networks are different in shape, with a sharper decline in the case of a scale-free network. Nevertheless we find that few nodes will cause damage as much as $10 \%$ of the whole system capitalization, while no bank in the random network will case a damage higher than 3.5\%, as seen in Figure 4.10. 

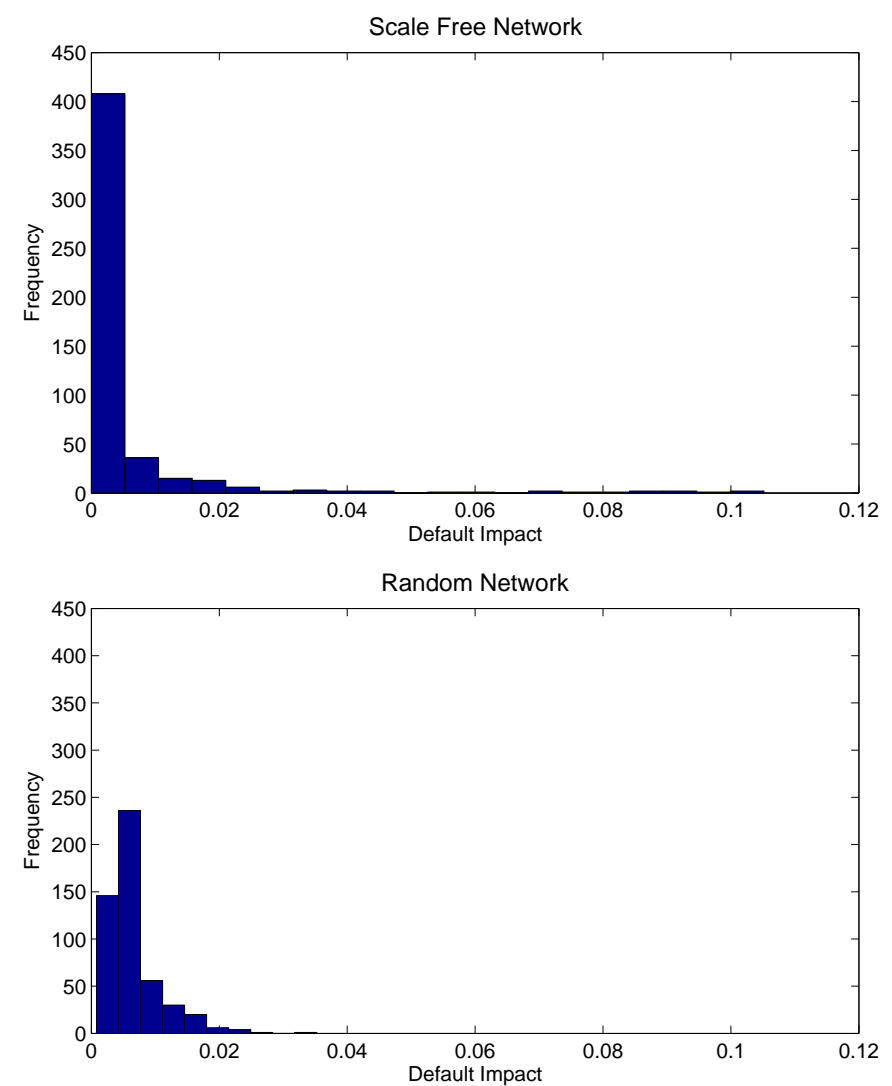

Figure 4.10: Histogram of default impact of a single bank default in scale-free and random networks.

In terms of the number of banks that have been triggered to default as a function of the in-degree of the initially defaulted bank, in the scale-free case we find that the number of defaulted banks is as high as 12, more than triple the number in the case of a random network. The effect of the highly connected banks is obvious from both Figure 4.11 (number of subsequent defaults) banks and Figure 4.12 (default impact). Highly connected banks cause the highest damage if compared to the less connected banks in the scale-free network. 

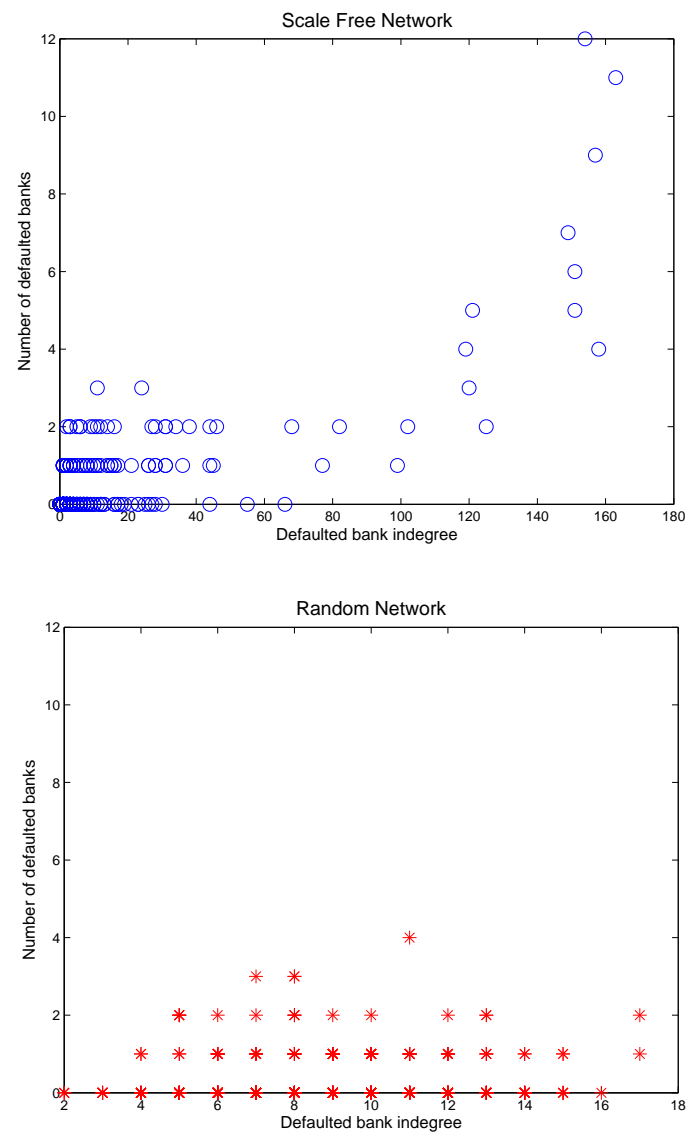

Figure 4.11: Relation between the in-degree of initially defaulted bank and the number subsequent defaults in scale-free (top) and random (bottom) networks. 

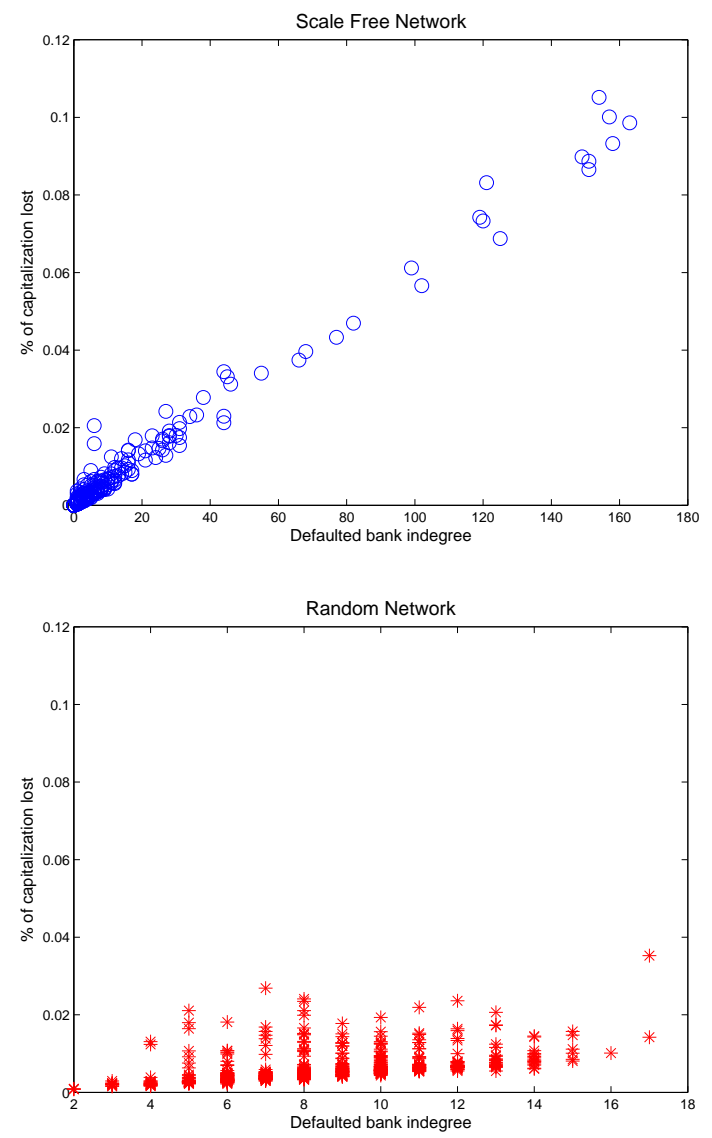

Figure 4.12: Relation between the in-degree of initially defaulted bank and default impact in scale-free (top) and random (bottom) networks.

While the examples above demonstrate the difference the network structure can have on its stability (in terms of percentage of capitalization lost and further number of banks triggered to default), our main aim is examining the stability of our network under the scale-free network model. The following section is devoted to a closer examination of the effect of highly connected banks. 


\subsubsection{Highly connected banks in a scale-free interbank net- work.}

From the simulation above, we observe that the most connected banks cause the most damage to the network under a single-bank default scenario. In a further examination to that observation we carried 500 independent simulations (using the same parameters as above) for a scale-free network of 500 banks. To examine the effect of these highly connected banks, we divided the banks in each of our networks into two sets: the top $2 \%$ highest connected banks (highest in-degree), and the rest of the banks. For each set, and for each simulated run of the network, we calculated the average in-degree, average default impact and average number of banks triggered to default. The results are shown in Figure 4.13.
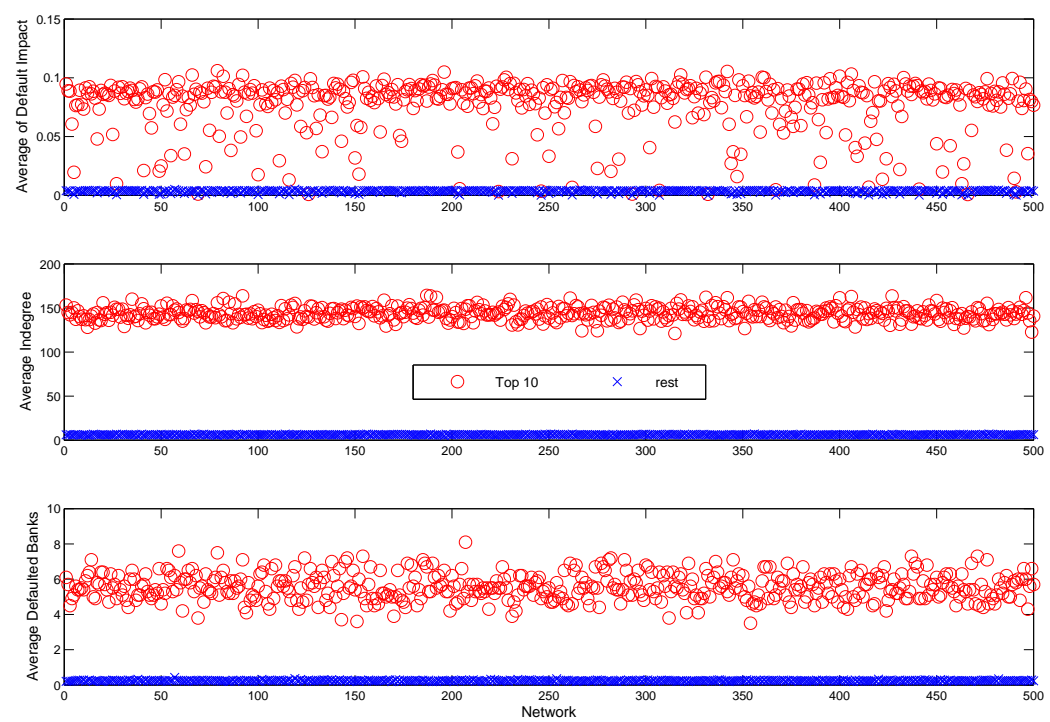

Figure 4.13: Results for 500 Simulated Networks. Top 10 connected banks compared to rest of the banks in a scale-free network of 500 .

As illustrated, there is a clear distinction between results for the two sets of banks. The first panel of Figure 4.13 indicates that on average a bank in the top 10 most 
connected will cause an average loss of $7.75 \%$ of capitalization for the system (corresponding to the average of the 500 simulated runs, with standard deviation of 0.02 ) compared to an average damage of $0.35 \%$ caused by a bank that is not in the top 10 (with standard deviation of 0.001), that is a top bank can cause a damage of 20 times more than that of a not highly connected bank. The second panel demonstrates the existence of hubs: for the first set the average is 143.8 in-degrees compared to 6.16 in-degrees for the rest of the banks (7.3 standard deviation for the first and 0.31 for the second). Finally the bottom graph of the figure shows that the initial shock of one of the top 10 most connected banks will cause an average of 5.57 other banks to default as a result, compared to 0.24 as an average for the second set of banks (standard deviation of 0.76 for top banks and 0.03 for the rest).

\subsubsection{The Systemic Risk Index}

Recall that the systemic risk index for bank $i$ is define in [CS09] as

$$
S I_{i}=E\left[D I_{i}^{\epsilon} \mid \bar{C}_{e 2, i}+\sigma_{i} \epsilon_{i}<0 .\right]
$$

To calculate the systemic risk index associated with banks in our networks, we started with a sequence of correlated uniform random variables $u_{1}, u_{2} \ldots, u_{n}{ }^{6}$ (correlation coefficient of 0.4 ) which in turn is used to generate the sequence of heavy tailed random variables $\epsilon_{1}, \epsilon_{2}, \ldots ., \epsilon_{n}$. Next we follow [CS09] and obtain $\sigma_{i}$ for each bank using their historical probability of defaults $\pi_{i}$ through the relation

$$
\sigma_{i}=-\frac{\bar{C}_{e 2, i}}{F^{-1}\left(\pi_{i}\right)} .
$$

\footnotetext{
${ }^{6}$ Following [CS09] to obtain a sequence of correlated uniforms through generating a sequence of $\left(n_{t}+1\right)$ IID standard normal $N(0,1)$ random variables $z_{0}, z_{1}, \ldots, z_{n}$. Applying gaussian copula with desired correlation coefficient $\rho$, we have that

$$
u_{i}=\Phi\left(\sqrt{\rho} z_{i}+\sqrt{1-\rho} z_{0}\right)
$$
}


For our simulated networks, we take the probabilities of default to be a uniformly distributed number between $0.01 \%$ to $9.65 \%$. This is motivated by Standards and Poor's 2009 Corporate transition rates for emerging markets. We assumed out of the 7 available rating categories, starting with AAA and ending with the worst rating of $\mathrm{CCC} / \mathrm{C}$, that Brazilian banks are somewhere between best rating of AAA and lastto-worst rating of $\mathrm{B}$. Thus corresponding to transition rates to default of $0.00 \%$ and 9.65\% for AAA rating and B rating respectively [SP09].

Starting with a 500 interbank network and using 1000 Monte Carlo simulations we obtained the following graph for the systemic risk index as a function of the in-degree of the defaulted bank.
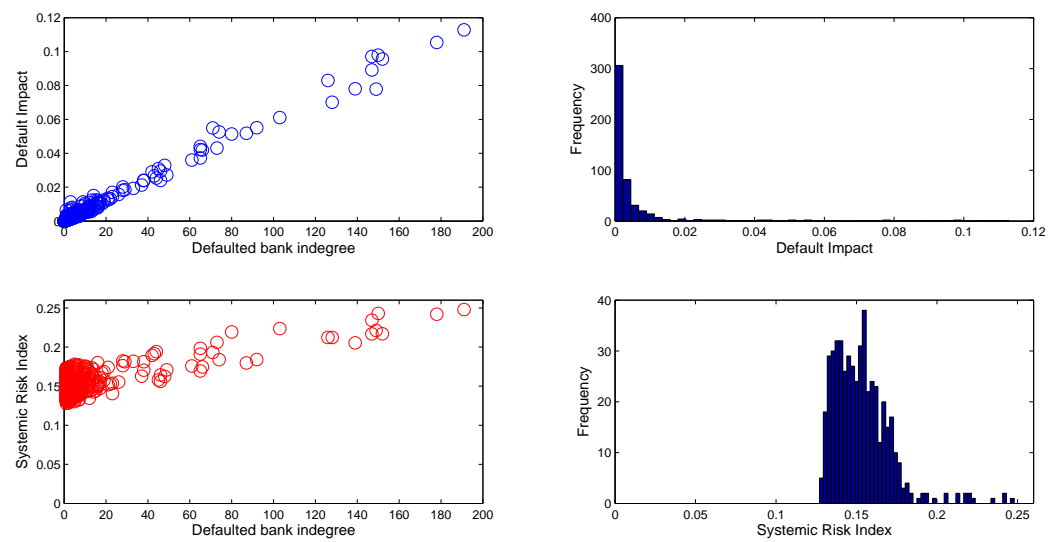

Figure 4.14: Results for 500 simulated networks, in a 1000 Monte Carlo runs.

As mentioned in [CS09] in the case of the Brazilian actual network, there are two main differences to be noted in comparing the systemic risk index with the default impact of a bank, which were also confirmed in our simulations for a network of 500 banks. First is the magnitude of the systemic risk index, which they reported to be relatively higher than that of the default impact (with an SI as high as $40 \%$ of the system capitalization). Similarly we observe $S I$ on average twice as high as the $D I$, reaching up to $25 \%$. Second, and more interestingly, they reported a positively skewed shape for the $S I$ distribution unlike the power law shape previously documented for 
Omneia R.H. Ismail - PhD Thesis - McMaster University - CES

the $D I$. We also observe the same for our 500-bank network, as shown from the histograms of the $S I$ and $D I$ on the right of Figure 4.14 .

\subsection{Conclusions and further directions}

In the first three chapters of this thesis, through a brief review of multiple empirical studies of the interbank market in various countries, we demonstrated how the structure of the interbank market is shown to follow that of a scale-free network. We introduced fundamental concepts and notions of modern network theory so as to facilitate our task of building a simulated network resembling that of empirically observed interbank networks. We built a weighted directed scale-free network, guided by the detailed empirical description of the Brazilian interbank market in Cont and Santos [CS09].

Our main goal was to examine through simulations the resilience and stability of the network and the implications of its topology. We implemented the default mech-

anism of [CS09] and measured the effect of single-bank failures using their measure of default impact. Our results confirm theirs in that most banks in the network will cause limited losses (not more than $4 \%$ of the system capitalization), but nevertheless a few banks are very risky and can cause as high as more than triple the amount of losses caused by other banks (as high as $15 \%$ of the system capitalization). We also showed how, in such a network, the connectivity of the defaulted bank related to its default impact and how many further defaults were triggered as a consequence to the initial defaults. In a clear positive relation between bank connectivity and its impact, we find that the higher the bank connectivity (in terms of in-degree) the greater the losses to be suffered in the system in terms of capitalization, which is to be expected in a scale-free network. We also demonstrated that the highest number of triggered to default banks is associated with the default of these highly connected banks.

In examining the effect of topology on stability and resilience, we built another 
simulated network serving as a comparative benchmark to the scale-free network, and for that we chose the random network topology given its well-known and distinctive properties (compared to scale-free). We enlarged our network to 500 banks for a better examination of the implications of the interbank topology. We confirmed, unlike the case of the random network, the existence of hubs in our scale-free network (banks with in-degree reaching up to about 165 compared to only 18 in the case of random networks) as to be expected with such topology. We find that while most banks in the scale-free network will not trigger much damage to the system if defaulted (robustness under random failures of nodes), few banks will cause a great damage (fragile in face of targeted attacks). Our work supports the documented differences in the resilience of the two network topologies reflecting the "robust-yet-fragile" nature of scale-free networks.

The highly connected banks were the ones responsible for the greatest damage to the system in terms of capitalization loss and additional numbers of banks triggered to default, particularly in the case of scale-free networks. The relationship between the defaulted banks connectivity is less apparent in the case of random networks. In terms of magnitude, the damage caused by the highly connected banks in the scalefree network reaches up to triple the maximum damaged witnessed in the random network.

Moving further in our analysis and highlighting the role played by these "too interconnected" banks, we built 500 independent scale-free networks each of 500 banks and measured the extent of damage caused by single-bank failures. We separated our banks according to connectivity as one group consisting of the top 10 connected banks and another with the remaining banks. The connectivity of such highly connected banks reaches up to 23 times that of the rest of the other banks on average (in terms of in-degree). We documented how on average the damage caused by one of the top connected banks measured by capitalization loss reaches up to 20 times that of other banks, and 25 times higher for the number of subsequently triggered defaults. 
We concluded this part of the thesis, by briefly examining the effect of systemic events on our networks following the work of Cont and Santos [CS09]. We subjected our network to exogenously correlated shocks affecting all banks through a Monte Carlo simulation, reaching similar conclusions to theirs in that few highly connected banks cause the most systemic damage to our network.

While the conclusion derived from such empirical and simulation-based studies help to shape our understanding of the nature of the banking system, they are essentially static and in our opinion provide a limited understanding of the system. The essence of such empirically based studies is a snapshot view on the nature of the network, which is then subjected to a mechanical chain reaction to the initial bank knockout default. In such studies banks do not learn, anticipate, or react to other banks default.

There is no doubt that additional features can be incorporated to such studies enriching their mechanisms. Nevertheless our claim is that by targeting the question of the effect of network topology on its stability as the one and only question such a stream of studies essentially lacked the interacting and shaping forces underlying the banking organization and replaced such forces by a set of simplifying assumptions. It is our belief that the study of systemic risk and contagion in a banking system is an integral part to the study of banking, bank runs and interbank formation.

In the remainder of the thesis we focus on the issues of banking, banks runs and the interbank formation and explore how in addressing these issues we essentially develop the forces for a dynamic banking network in an agent-based modeling framework. 


\section{Chapter 5}

\section{Bank formation and bank runs}

This chapter constitutes the beginning of the second part of our analysis, in particular the introduction of our agent based model. As stated before, our aim is to derive the necessary forces for a dynamical setup of the interbank market. We start with individuals in a pre-banking society and illustrate how their liquidity needs give rise to banking and also to bank runs. In later chapters we show how these forces evolve giving rise to the interbank market. We start our chapter by introducing the Diamond and Dybvig model [DD00] as a widely used model to understand bank runs and other types of financial crisis. Unlike their model, instead of assuming individuals always join the banks seeking liquidity, we present our society initially with no banks and illustrate how individuals' heterogeneous needs gave rise to banks. When considering bank operation and the possibility of facing bank runs, we use the same intuition as that of Diamond and Dybvig but adapted to our agent-based model without any a priori equilibrium assumption, infinite foresight, or a central planner.

\subsection{The Diamond and Dybvig Model}

In the early 1980s, Diamond and Dybvig developed a model [DD00] illustrating why banks choose to issue deposits that are more liquid than their assets and why for 
the same reason banks are subject to runs. According to their model, individuals have a natural demand for liquidity as they are uncertain about when they need to consume and as such for how long they want to hold assets. It provided the first explicit analysis for the liquidity transformation service provided by banks as well as why individuals choose to join banks.

The model has 3 times $(t=0,1,2)$, a single homogeneous consumption good and a productive technology (the illiquid asset) yielding a payoff of $R>1$ units of output (consumption good) at time $t=2$ per unit invested at time $t=0$. On the other hand, if the productive technology is liquidated at $t=1$ it yields just the initial investment. In addition, the consumption good can be stored at no cost and therefore serves as a numeraire having the same value at all times (the liquid asset). In other words, for each unit invested at time $t=0$, the illiquid asset has a payoff structure $(1, R)$ at times $t=1,2$, whereas the liquid asset pays $(1,1)$. An individual is called an investor of "type 1" (an impatient agent) if he needs to consume at the intermediate time step $t=1$, otherwise he is called an investor of "type 2" (a patient agent). At time $t=0$, each investor is given one unit of endowment, but does not know which type he will be at subsequent times. That is, each investor faces a privately observed risk of being type 1 or type 2 with probabilities $w$ and probability $(1-w)$. There is no aggregate uncertainty in the society: a fraction $w$ of individuals will be type 1 investors.

In a simple utility maximization framework, Diamond and Dybvig demonstrated that in a situation where the individuals hold the asset directly, and given the possibility of trading in the form of a competitive market in claims on future goods, there will never be any trade and individuals will invest only for their own consumption. That is, if we denote by $c_{k}^{i}$ to the optimal consumption level at period $k$ of an agent of type $i$, it then follows that $c_{1}^{1}=1, c_{2}^{1}=c_{1}^{2}=0$, and $c_{2}^{2}=R$. The results follow from the fact that investors are identical at the beginning and their types are privately observed risk, accordingly there can be no optimal risk-sharing between them.

On the other hand they showed if their types were publicly observable, it is possi- 
ble to write an optimal insurance contract that gives the ex ante (as of period $t=0$ ) optimal sharing of output between the two types of investors with optimal consumption levels satisfying $c_{1}^{1 *}>1$ and $c_{2}^{2 *}<R$. Such a contract will allow individuals to insure against the unfortunate outcome of prematurely liquidating their investments. Nevertheless such a contract cannot be offered in a simple contingent claims market, since liquidity preferences are not publicly observable. Fortunately, the optimal risk sharing solution satisfies a self-selection condition that ensures that there exists a contract that realizes it as a Nash equilibrium even for privately observed risks. This is the cue for the explicit economic role of banks in offering such a contract.

In their model, a bank constructs a demand deposit contract offering each agent withdrawing at the intermediate time step $(t=1)$ a payoff of $r_{1} \geq 1$ per unit deposited. Withdrawals are served sequentially in random order, until the bank runs out of funds. They assumed the bank is mutually owned and the payoff for the type 2 investors is obtained by liquidating the bank in period 2, with each type 2 depositor receiving a pro-rated share of the bank's assets.

Let $V_{k}$ be the $k$-period payoff per unit of deposit withdrawn, which for $t=1$ depends on the person's place in the line of withdrawing people. Let $f_{j}$ be the fraction of withdrawers' deposits served before depositor $j$, and $f$ be the total quantity of withdrawn deposits. It then follows that:

$$
V_{1}\left(f_{j}, r_{1}\right)= \begin{cases}r_{1} & \text { if } f_{j}<\frac{1}{r_{1}} \\ 0 & \text { if } f_{j} \geq \frac{1}{r_{1}}\end{cases}
$$

and

$$
V_{2}\left(f, r_{1}\right)=\max \left\{\frac{R\left(1-r_{1} f\right)}{(1-f)}, 0\right\} .
$$

They showed that such a contract has one of its equilibrium as an optimal risksharing and occurs when $r_{1}=c_{1}^{1 *}$ and $f=w$. However, another equilibrium is a bank run with $f=1$ : if most agents anticipate that a large fraction will withdraw at time $t=1$ then all agents will actually prefer to withdraw at $t=1$. This happens for all 
demand contracts with $r_{1}>1$ (note that for $r_{1}=1$ there would be no need for banks as that would be like mimicking direct holding of the assets). They concluded that "a demand deposit contract which is not subject to runs provides no liquidity services".

The remedy they proposed is a deposit insurance, which is typically offered by the government and guarantees that the promised return on the deposits will be paid to all individuals who withdraw. Since its introduction their model has been widely used to understand banks runs (see for example [AG98]). In the next section, and before adapting the Diamond and Dybvig model for banks in our setting, we illustrate the case of individuals facing the investing decisions on their own in what we call the "prebanking" society. We demonstrate how in such societies individuals who are subject to preference shocks can learn to anticipate their future investment preferences and situation. Later on we show how this learning capability gives rise to the need for banking.

\subsection{The pre-banking society}

We start with a society consisting of group of individuals arranged in a 2-dimensional cellular automaton, an economy consisting of one liquid and one illiquid traded asset, and time being represented as a series of periods. At the beginning of each period, individuals receive one unit of endowment and make their investment decisions based on their preference. At the mid-period, preferences are subject to shocks according to which they might change for some individuals. Individuals who changed their preferences will then search their neighbourhood seeking trading partners so as to satisfy their altered new preferences. The uncertainty of finding the partners coupled with the expectation of future preference shocks motivate individuals to look for the more stable outcome offered by banks, even with lower payoffs than could be obtained by trading partners in most cases. 


\subsubsection{Individuals, preferences, and investment choices}

Our society has $N$ individuals, each given a predominant (innate) preference according to independent uniform random variables $U^{i}$ on $[0,1]$ : if $U^{i}<p$, agent $i$ is deemed to be a type 1 investor (impatient), otherwise the individual is a type 2 investor (patient). Time is initially represented by a single period with instants labelled by $t=0,1,2$, and the economy consists of two assets (liquid and illiquid) whose payoffs at times $t=1,2$ for each unit invested at time $t=0$ are given by

\begin{tabular}{|c|c|c|}
\hline Assets & payoff at $t=1$ & payoff at $t=2$ \\
\hline liquid & 1 & 1 \\
illiquid & $r$ & $R$ \\
\hline
\end{tabular}

with $r<1<R$.

At $t=0$, each individual receives one unit of consumption good (serving as numeraire) which we might think of as uniform wages. Based on their innate preferences, they make their initial investing decisions: impatient agents invest in the liquid asset, patient agents invest in the illiquid one. At $t=1$, all individuals are subject to independent privately observed preference shocks according to

$$
\varrho^{i}=U^{i}+(-1)^{b^{i}} \frac{\epsilon^{i}}{2}
$$

where the shock strength $\epsilon^{i}$ is a uniformly distributed random variable on $[0,1]$, and its direction $b^{i}$ is a Bernoulli distributed random variable on $\{0,1\}$ (with success probability equals to 0.5$)^{1}$. Setting $q=2 p-1 / 2$, individual $i$ is then deemed to be impatient as a result of the shock if $\varrho^{i}<q$, and patient otherwise. For the reminder of our work, we fix $p=1 / 2$ for concreteness, knowing that any other fraction could be considered as well.

\footnotetext{
${ }^{1}$ Note that while $U^{i}$ is fixed for each individual across time, the rest of our variables (e.g., $b^{i}$ and $\epsilon^{i}$ ) are independent from each other as well as from $U^{i}$ and are indepedent accross time and accross different individuals.
} 
The rationale behind this setup for preferences is that we assume individuals are heterogeneous and for the most part conscious of what they want initially, yet there is a chance that they might change their mind later on. Accordingly, if the mid-period shock is big enough, some individuals will have their preferences altered and would wish to have different investment. This possible discrepancy between initial investment decision and subsequent needs motivates individuals to explore their society searching for partners to exchange investment.

\subsubsection{Searching for partners}

In modelling our society we needed to impose some constraints on the individual capacity to go around and seek other individuals to trade reflecting the inherited limited capability of information gathering and environment knowledge of individual agents. As such we use the Cellular Automata (CA) framework as a simple and natural way to limit the individual knowledge to only local interaction.

Individuals are arranged over a two dimensional CA, and each cell is assumed to have 8 neighbours which it can interact with. Here we use a combination of Von Neumann and Moore neighbourhood: the individual becomes aware of his four orthogonal neighbours first (i.e Von Neumann), then of his remaining 4 diagonal neighbours (remaining of Moore neighbourhood). To add more structure to our model we use the following sequence according to which the person becomes aware of his neighbours:

$\begin{array}{lll}5 & 1 & 6 \\ 2 & \mathrm{X} & 3 \\ 7 & 4 & 8\end{array}$

Now at $t=1$, due to the preference shock, there would be some individuals who made their initial investment and need to switch them, if possible, by finding a trading partner. These individuals will announce themselves, and each person in need would know which of their neighbours is searching for liquid or illiquid assets. People 
who after the shock still do not want to change their initial investment, because the shock was not strong enough to alter their preferences, will be like mute cells whose neighbours do not know what kind of investments they made. The situation resemble a marketplace where only people needing to trade are those that declare themselves.

We then randomly pick a cell at a time and match it with its closest neighbour according to the table above, and keep going until all cells that could be matched are matched. After all matching has taken place, there might still be some cells in need of partners who could not find any. In Figure 5.1 we illustrate this procedure in a society of 100 individuals $(10 \times 10$ grid $)$. The top panel shows the initial preferences of individuals (green representing patient agents, and red cells representing impatient ones). The middle then shows how preferences had changed for some individuals due to the independent preference shocks, with blue cells representing individuals that did not change their preferences, while green and red cells show the new preferences for those who changed and are therefore in search of trading partners. Finally the bottom panel represents the society after the matching procedure described above has taken place. As indicated there are still some individuals that need partners yet could not find one. Later on we show how the existence of these individuals gives rise to banks. 
(1)

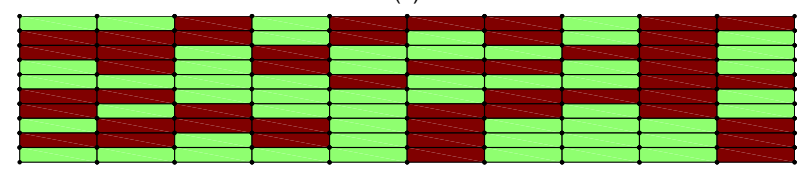

(2)

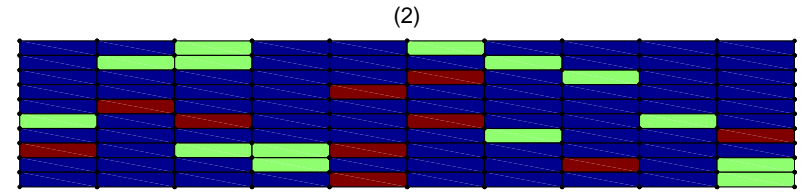

(3)

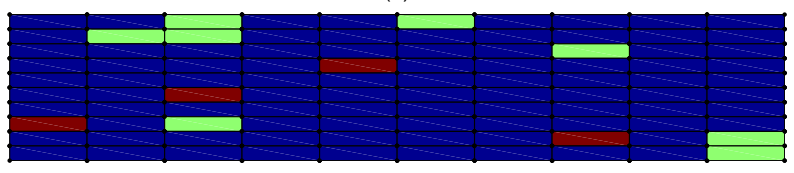

Figure 5.1: Society, preference shock, and the search for partners.

\subsubsection{Learning and predicting}

Now consider a repeated version of our model with times $t_{0}, t_{1}, t_{2}, \cdots, t_{2 n}$ divided into periods $\left[t_{2 k}, t_{2 k+2}\right]$ for $k=0,1,2, \cdots, n-1$. At the beginning of each period, that is at times $t_{2 k}$, the preferences of individuals is set as equal to the initial preference determined at $t_{0}$ by the random variables $U_{i}$ as described in Section 5.2.1. As before, at the middle of each period, that is at $t_{2 k+1}$, individuals are subject to the preference shock $\left(b_{k}^{i}, \epsilon_{k}^{i}\right)$ that temporarily alter their preferences according to (5.1). Individuals again have to invest in a liquid and illiquid asset with the same payoffs as before, which are now realized at $t_{2 k+1}$ and $t_{2 k+2}$ instead of $t=1,2$.

As the shocks are drawn afresh in each period, the interesting question that follows is how individuals can predict what will happen to them in the next period, knowing their innate preferences and the effect of random shocks. More specifically, whether an individual will need a partner and, if so, can find one. In trying to answer this question we follow the seminal work of Brian Arthur in inductive reasoning [Art94, Art92]. As he presented in his El-Farol problem, individuals have bounded 
rationality and in dealing with ill-defined problems they employee inductive thinking, through building a representation of the situation they are facing, recognizing patterns, forming hypothesis about the environment and strengthening or weakening the confidence in the hypothesis as more evidence become available, individuals can then successfully deal with different problems that deductive reasoning falls short in dealing with [Art94].

In inductive reasoning, agents typically use two types of mechanisms. The first type, known as Learning Classifier Systems, consists of searching for patterns through mapping the information from previous periods into predictors for the situation in the coming period, and as experience builds in updating the strength of the predictors [BGH89]. The second type consists of developing new predictors by modifying, combining and mutating existing predictors, through a mechanism like the Genetic Algorithm.

As a start, we use a simple adaptive mechanism that in essence follows the learning classifier system, and use only the first type of mechanism of employing past information to predict future outcomes. We show that even with this simple structure agents for the most part learn and can successfully predict the next period situation they would be facing. We assume agents have a limited memory of 5 periods, according to which they try to make a prediction for the next period. We endow all agents with a homogeneous set of 7 predictors as follows:

1. This period will be the same as the previous one.

2. This period will be the same as two periods ago.

3. This period will be the same as three periods ago.

4. This period will be the same as four periods ago.

5. This period will be the same as five periods ago.

6. This period will be the same as the mode of the last three periods. 
7. This period will be the same as the mode of the last five periods.

Each predictor makes one of the following three forecasts about the situation to be faced in the next period: a neutral forecast $(N)$, meaning that the agent will not change preferences, a good forecast $(G)$, meaning that the agent will change preferences and find a trading partner, and a bad forecast $(B)$, meaning that the agent will change preferences and fail to find a trading partner. Each person starts with all seven predictors having the same strength of zero. As time goes by, the strength of each predictor is updated by adding one unit for each correct forecast and subtracting one for each incorrect one. Agents then use the predictor with the highest strength to predict the situation in the next time period.

Applying our method to a society of 400 persons over a time span of 100 periods in a Monte Carlo simulation of 100 independent societies, the average percentage of agents with correct predictions increases in time (time in periods represented on the $x$-axis), reaching up to $68 \%$, as shown in Figure 5.2, with the left panel showing all 100 Monte Carlo paths and the right one showing their average.
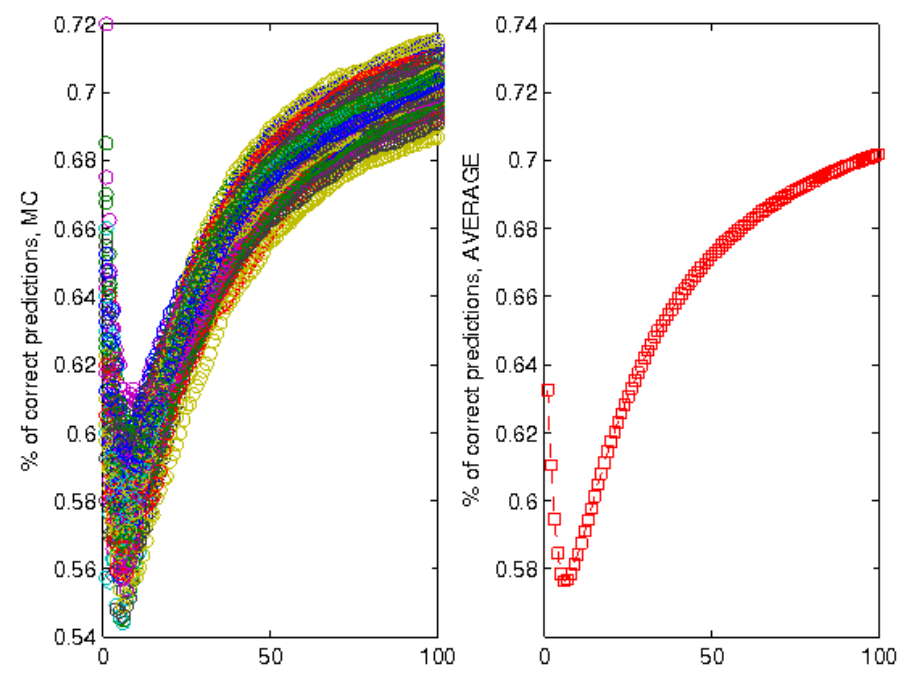

Figure 5.2: Percentage of correct predictions.

We expect the performance to increase if more sophisticated predictors are to be 
Omneia R.H. Ismail - PhD Thesis - McMaster University - CES

used, particularly if a genetic algorithm to generate new predictors is employed. In any event, we see that the majority of the individuals make correct predictions about their need to find trading partners and their success in doing so. Those individuals that predict a bad outcome therefore have a strong incentive to look for a different type of agent, one offering a reasonably attractive contract in the event of a change in liquidity preferences, thus creating a natural demand for banks to be established.

\subsection{Bank formation}

We demonstrated that when individuals are subject to preference shocks, there will be some of them who needed trading partners and unfortunately could not find one. In addition we showed that with time individuals learn about the possibilities of needing and finding partners and can predict (to some extent) their future situation. We argue that this is the motivation giving rise to banks, that is, banks are formed as individuals learn that they can face the unfortunate case of needing yet not finding trading partners.

\subsubsection{Joining a bank}

Let us assume for the moment that a bank exists in the neighbourhood of an agent and promises payoffs $\left(c_{1}, c_{2}\right)$ satisfying $1<c_{1}<c_{2}<R$ for withdraws at $t_{2 k+1}$ and $t_{2 k+2}$, respectively, for each unit deposited at $t_{2 k}$. For individuals making predictions for the situation in the next period, under what conditions would a person prefer to join a bank, assuming that a bank run is not yet an issue to be considered by individuals? We have the following possibilities:

1. An impatient agent predicting to not change preferences in the coming period will always join the bank, since the payoff promised by the bank is always greater than the payoff from directly investing in the liquid asset $\left(c_{1}>1\right)$. 
2. An impatient agent predicting to change preferences and to fail to find a partner in the coming period will always join the bank, since the promised payoff is higher than the payoff of holding one unit from $t_{2 k+1}$ to $t_{2 k+2}\left(c_{2}>1\right)$.

3. A patient agent predicting to change preferences in the next period will also join the bank whether he expects to find a partner or not, since the payoff is higher than that from of exchanging with a partner $\left(c_{1}>1\right)$.

4. An impatient agent predicting to change preferences and to find a partner in the coming period will not join the bank, since clearly his payoff from trading is higher than the bank promised payoff at $t_{2 k+2}\left(R>c_{2}\right)$.

5. Finally a patient agent who is confident in not changing preferences in the next period will not join the bank, since his expected payoff is higher than the promised one from the bank $\left(R>c_{2}\right)$.

If we add to our structure that once joined a bank a person stays in, the bank is an absorbing state and eventually will likely include most individuals as its clients in the long run, a hypothesis we put to test.

In a simple framework we could say that individuals join the bank if their highest strength predictor forecasts they will be one of the first three types indicated above. Such a simple setup assumes that individuals can correctly predict the state of the next period and make "quick" decisions. However, given the predictions are not entirely accurate, we argue that the act of deciding whether or not to join the bank requires further "thinking" on the part of individuals, carefully assessing the different possibilities.

Our differentiation between the two modes of deciding (quick versus deliberate thinking) given the uncertainty of the future is expressed in the work of Nobel prize laureate Daniel Kahneman [Kah11]. What we refered to as "quick" represents what he calls System 1 of the mind, which among other characteristics generates quick impressions, feelings and inclinations with little or no effort, neglects ambiguity and 
suppresses doubt. On the other hand, he introduces the notion of System 2, the more conscious mode of thinking that gets activated to deal with ambiguous situations or these requiring effort and involving nontrivial matters. As Kahneman puts it "uncertainty and doubt are the domain of System 2".

We therefore propose a mechanism for each individual to combine his predictors and compare the payoffs obtained by not joining the bank (that is investing directly in the two assets) against the payoff promised by the bank, according to his current state of forecasts and weighted by the strength of each of his predictors.

To illustrate our mechanism, consider a patient agent whose predictors have the forecasts and strengths shown in the first two columns of the table below:

\begin{tabular}{rrrcc} 
& Forecast & Strength & payoff (join) & payoff (not join) \\
\hline 1 & $\mathrm{~N}$ & -2 & $c_{2}$ & $\mathrm{R}$ \\
2 & $\mathrm{G}$ & 0 & $c_{1}$ & 1 \\
3 & $\mathrm{~N}$ & +1 & $c_{2}$ & $\mathrm{R}$ \\
4 & $\mathrm{~B}$ & -1 & $c_{1}$ & $\mathrm{r}$ \\
5 & $\mathrm{G}$ & +1 & $c_{1}$ & 1 \\
6 & $\mathrm{~N}$ & 0 & $c_{2}$ & $\mathrm{R}$ \\
7 & $\mathrm{~B}$ & +2 & $c_{1}$ & $\mathrm{r}$
\end{tabular}

where $N, G$ and $B$ stand respectively for the neutral, good and bad forecasts discussed in Section 5.2.3. The last two columns represent the agent's payoffs for the two cases of joining and not joining the bank. According to the first predictor, the agent is better off not joining the bank since $R>c_{2}$, while according to the last predictor he is better off joining the bank $c_{1}>r$. Individuals weight each of their predictors' payoff with the strength of the corresponding predictor and decide whether to join or not to join according to which option has the largest weighted payoff. For example, using $c_{1}=1.1, c_{2}=1.5, r=1, R=2$ leads to the decision of joining the bank as joining the bank has a weighted sum of 0.7 versus that of not joining the bank with weighted payoff sum of 0 . 
Before moving forward, we note that example above is a case where the use of System 1 and System 2 would lead to the same conclusion. Using only the predictor with the highest strength (predictor 7) leads to the same decision of joining the bank of the weighted payoff sum since $c_{1}>r$. That need not be always the case, since changing the strength of the first predictor from -2 to +1 does not change System 1 decision of joining the bank, yet alters System 2 decision of not joining (weighted average of 5.2 for joining the bank against 6 for not joining).

So far we discussed the individuals' story and considered their choices given there are banks which promised certain payoffs. Next we consider the bank's story, adding another layer of hierarchy to our agent based model of the individual investors. We discuss what might motivate the establishment of banks in what we call bank birth, how they operate, and under what conditions they face liquidity problems and termination.

\subsubsection{Bank birth}

We modelled the birth of a bank in an agent-based model as an emergent phenomenon following the steps of Howitt and Clower [HC00] and Howitt [How08].

At the beginning of each period $t_{2 k}$, an agent is randomly chosen to decide whether or not to establish a bank. Upon being hit by this "idea of entrepreneurship", the person looks around in his local environment to see if any of the 8 neighbours is already bank, in which case he will not establish one. If not, the agent tries to estimate the number of patient and impatient investors amongst his immediate 9 potential clients (including himself). Let us denote the fraction of early customers estimated by person $i$ at the beginning of the period $[2 k, 2 k+2]$ by $W_{k}^{i}$, initially estimated as a random drawn from the set $\{0 / 9,1 / 9, \cdots, 9 / 9\}$ (with equally probabilities), which represents the "Animal Spirits" in [How08], that is, "a tangled melange of hope, fear, and luck". Later, we equip banks with a leaning mechanisms to update their initial estimate.

Based on the estimate $W_{k}^{i}$, he determines whether it is feasible for him to establish 
a bank promising a payoff $c_{1}$ for early withdrawals and $c_{2}$ for late withdrawals, such that $1<c_{1}<c_{2}<R$ (it should be clear by now that if the bank offered otherwise, individual investors would have no incentive to join). We assume throughout that $c_{1}$ and $c_{2}$ are given exogenously.

Similar to the other individuals, the person who is hit by the idea to form a bank can invest in the liquid and illiquid assets. Following the notation in Allen and Gale [AG98], let $x_{k}^{i}$ denotes the proportion of potential deposits invested in the illiquid asset and $y_{k}^{i}$ to be the proportion invested in liquid asset. The agent will establish a bank if he can find $x_{k}^{i}$ and $y_{k}^{i}$ such that $x_{k}^{i}+y_{k}^{i} \leq 1$ and satisfying

$$
\begin{aligned}
y_{k}^{i} & =c_{1} W_{k}^{i} \\
R x_{k}^{i} & =c_{2}\left(1-W_{k}^{i}\right) .
\end{aligned}
$$

If it happens that $x_{k}^{i}+y_{k}^{i}<1$, then the fraction $\left(1-\left(x_{k}^{i}+y_{k}^{i}\right)\right)$ is deemed to be invested in the liquid asset and held by the bank as a reserve.

The agent then offers a demand deposit with payoffs $\left(c_{1}, c_{2}\right)$ to its immediate neighbours, who in turn decide whether or not to accept it following the mechanism described in Section 5.3.1. In addition we give the bank the reach of its clients: at each time step the bank offers deposits to agents in the neighbourhood of client who joined in the last period.

We assume for now that a bank is an absorbing state, whereby once a person joined he would not leave the bank, so that it is not subject to a run. Needless to say, under such an unrealistic assumption, once banks are established sooner or later most individuals will belong to banks. For example, using the parameters $c_{1}=1.1$, $c_{2}=1.5, R=2$ and $r=0.5$ if liquidated, we trace the establishment of banks in a society consisting of 6400 individuals ( $80 \times 80$ society) over a time of 80 periods and show the result in Figure 5.3, where we can see a case of multiple banks expanding the society (each highlighted with different colour) where most of the individuals (about 
$78 \%$ ) in the society joined banks at the end.

In the next section we consider the more general case where banks face the consequences of their own investments decisions and the possibilities of failures with the misestimation of their depositors' needs. Vulnerable to runs, banks need to learn and adapt to survive.

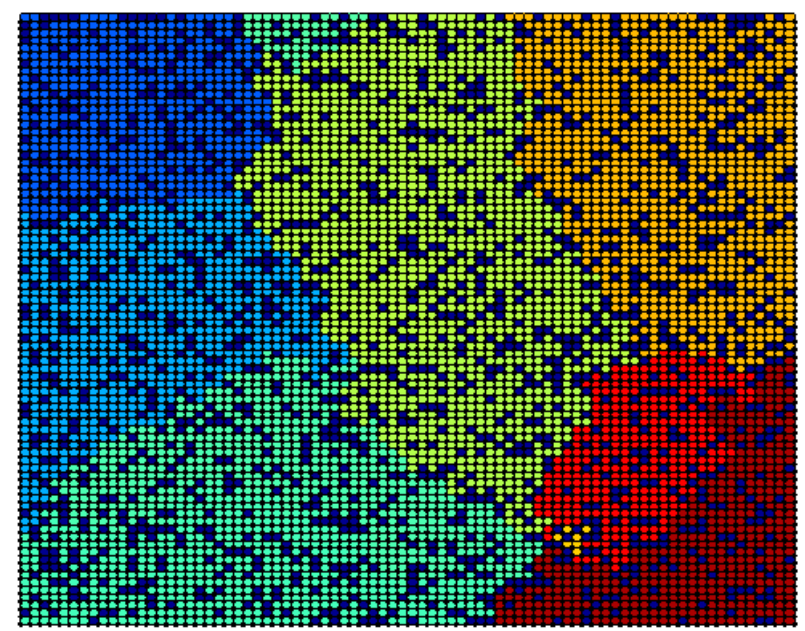

Figure 5.3: Banks established after 80 periods in a society of 6400 individuals, without the possibility of a bank run.

\subsection{Bank runs}

In the Diamond and Dybvig model [DD00], as well as many models following their footsteps, a bank run occurs when the face value of the deposits becomes higher than the expected value of waiting to withdraw at a later time. Specifically, the bank run is an equilibrium state in which all depositors (impatient as well as patient agents) withdraw at the intermediate time step because they expect all the others to do the same. Conditional on such expectation, the face value of the deposits for patient individuals is higher than the expected value of waiting to withdraw at next time 
step. Accordingly not only impatient depositors withdraw at the intermediate time step (as to be expected by the bank), but so do patient depositors, causing a run in the bank.

We now consider a more realistic version of our model where banks face the possibility of failure using the same intuition as that of Diamond and Dybvig, but adapted to our agent-based methodology.

\subsubsection{Bank survival problem}

Once a bank chooses their estimated proportion of early customers $W_{k}^{i}$ and makes the resource allocation at the beginning of the investing period $t_{2 k}$ according to 5.3, it faces the actual ratio $\overline{W_{k}^{i}}$ of impatient individuals at $t_{2 k+1}$. Two scenarios can therefore happen:

1. Underestimating $\left(\overline{W_{k}^{i}}>W_{k}^{i}\right)$ : If the actual proportion is higher than the one based on which the bank made its investment, the bank faces a liquidity problem. Denoting by $N_{k}^{i}$ the number of customers the bank has at period $k$, it needs to raise an amount equal to $c_{1}\left(\overline{W_{k}^{i}}-W_{k}^{i}\right) N_{k}^{i}$ to meet the excess demand for liquidity at time $t_{2 k+1}$. For this, the bank first attempts to use its own accumulated reserves (denoted as $R_{k}^{i}$ ). If these are enough to satisfy all early customers, that is, if $R_{k}^{i}>c_{1}\left(\overline{W_{k}^{i}}-W_{k}^{i}\right) N_{k}^{i}$, then the bank finds itself in the fortunate situation of having less late customers than previously anticipated. The amount left over is then added to the realized reserves for period $k$, that is,

$$
\overline{R_{k}^{i}}=\left(R_{k}^{i}-c_{1}\left(\overline{W_{k}^{i}}-W_{k}^{i}\right) N_{k}^{i}\right)+c_{2}\left(\overline{W_{k}^{i}}-W_{k}^{i}\right) R N_{k}^{i} .
$$

On the other hand, if the accumulated reserves are not enough to cover the shortfall at time $t_{2 k+1}$, that is if $R_{k}^{i}<c_{1}\left(\overline{W_{k}^{i}}-W_{k}^{i}\right) N_{k}^{i}$, the bank moves to the second and less favourable option of liquidating part of its illiquid assets. However, the maximum the bank can generate this way is limited by what 
needs to be paid to late customers. While it is clear that these might end up receiving less than the promised amount $c_{2}$ per unit invested, we impose that they should not receive less than $c_{1}$. Otherwise patient customers will receive less than what they could receive by withdrawing their deposits immediately at $t_{2 k+1}$. We assume that if this happens patient agents will punish the bank by withdrawing all their deposits at the first available opportunity, causing a bank run.

Therefore, the maximum amount gained from liquidation of the long term asset without causing a run is

$$
G\left(W_{k}^{i}\right)=\left[\frac{\left(1-W_{k}^{i}\right) c_{2}}{R}-\frac{\left(1-\overline{W_{k}^{i}}\right) c_{1}}{R}\right] N_{k}^{i} r
$$

where the first component in brackets expresses the payoff from the amount originally invested in the illiquid asset (based on the estimated $W_{k}^{i}$ ) and the second component expresses the minimum amount that has to be kept invested in the illiquid asset to appease patient customers. As such, a bank run occurs whenever

$$
c_{1}\left(\overline{W_{k}^{i}}-W_{k}^{i}\right) N_{k}^{i}>G\left(W_{k}^{i}\right)+R_{k}^{i} .
$$

In case of a bank run, we assume that its depositors go back to the society and their search for trading partners. In addition if there is a bank in reach for them, we impose one day recovery after their previous bank failure before any of them can join or establish a new bank.

2. Overestimating $\left(\overline{W_{k}^{i}}<W_{k}^{i}\right)$ : If the actual proportion of early consumers is lower than estimated, then all impatient customers are fully paid, whereas the bank's investment in the illiquid asset is in shortage to pay the full promised payment $c_{2}$ to patient customers. The total amount available to pay patient 
consumers is

$$
\begin{aligned}
c_{2}\left(1-W_{k}^{i}\right) N_{k}^{i} & +c_{1}\left(W_{k}^{i}-\overline{W_{k}^{i}}\right) N_{k}^{i} \\
& =c_{2}\left(1-\overline{W_{k}^{i}}\right) N_{k}^{i}-\left(c_{2}-c_{1}\right)\left(W_{k}^{i}-\overline{W_{k}^{i}}\right) N_{k}^{i}
\end{aligned}
$$

where the first term of the left hand side is the amount obtained from investment in the illiquid asset (based on the estimated impatient proportion) and the second term is amount carried over after paying impatient customers at $t_{2 k+1}$. Rearranging our terms as shown on the right hand side of the expression, the first term is the promised payment of $c_{2}$ to each customer, whereas the second term represents the amount by which this payment has been reduced as a result of overestimating early customers and accordingly underestimating the proportion of late customers. While we assume that patient customers are not a threat for the bank failure as long as they receive a payoff greater than $c_{1}$, we impose that banks use their accumulated reserves $R_{k}^{i}$ (if any) to try to reduce the loss expressed in the second term in the right hand side above.

To summarize, underestimating the actual proportion of early customers might lead to a bank run but could also increase the bank's reserves, whereas overestimating it is a safe strategy for the bank because it never leads to a run, but does not lead to increased reserves either.

\subsubsection{Banks learning and allocation problem}

Treating banks as a second hierarchy of agents in our analysis leads us to develop a learning mechanism for them. Having observed the actual ratio of impatient clients $\overline{W_{k}^{i}}$, we assume that banks that do not suffer a run during this period modify their estimate for the next period according to

$$
W_{k+1}^{i}=W_{k}^{i}+\alpha\left(\overline{W_{k}^{i}}-W_{k}^{i}\right)
$$


where $0<\alpha<1$ represents the speed of adaptation or learning. This is equivalent to assuming that banks use an Exponential Moving Average (EMA) model with a constant smoothing factor $\alpha$ to update their estimates.

The allocation for bank $i$ at time $t_{2 k+2}$ is simply as before

$$
\begin{aligned}
& y_{k+1}^{i}=c_{1} W_{k+1}^{i} \\
& x_{k+1}^{i}=c_{2}\left(1-W_{k+1}^{i}\right) / R .
\end{aligned}
$$

Observe, however, that $W_{k+1}^{i}$ might lead to an allocation violating the constraint $x_{k+1}^{i}+y_{k+1}^{i} \leq 1$. We therefore use

$$
W_{k+1}^{i}=\max \left\{W_{k}^{i}+\alpha\left(\overline{W_{k}^{i}}-W_{k}^{i}\right), \frac{1-\frac{c_{2}}{R}}{c_{1}-\frac{c_{2}}{R}}\right\}
$$

As before, if it happens that $x_{k+1}^{i}+y_{k+1}^{i}<1$ the difference is deemed to be invested in the liquid asset and becomes part of the accumulated reserves for the bank, which are then given by

$$
R_{k+1}^{i}=\overline{R_{k}^{i}}+\left(1-x_{k+1}^{i}-y_{k+1}^{i}\right) N_{k+1}^{i} .
$$

Given these rules for determining the survival, learning, and dynamic allocation for banks, we can iterate our algorithm over many periods and observe their evolution. As an example, Figure 5.4 shows six scenarios using the same parameter values as before and $\alpha=0.7$ fixed to all banks. Over the course of 100 periods we find situations where few banks emerged and successfully expanded to include most of the individuals in the society. In the first graph (1) we find fours banks successfully covering the society (first bank with 3788 members, second bank with 3002 members followed by third bank with 1462 members and the smallest one with 648 members).

On the other extreme, we find two cases where no bank could successfully establish itself. In graph (2) we have two banks with only 13 and 3 members, and in graph (3) we find just one bank with only 6 members. Yet in graph (4) we find another case 
where a single bank gained an almost monopoly over the society (8054 members) and a much smaller bank with only 6 members. In the last two graphs, we find that two banks established themselves in graph (5) having one giant bank (7826 members) and another significantly smaller one (534 members), while in graph (6) the two banks seems to be close size in size (4304 and 3919 members, respectively).
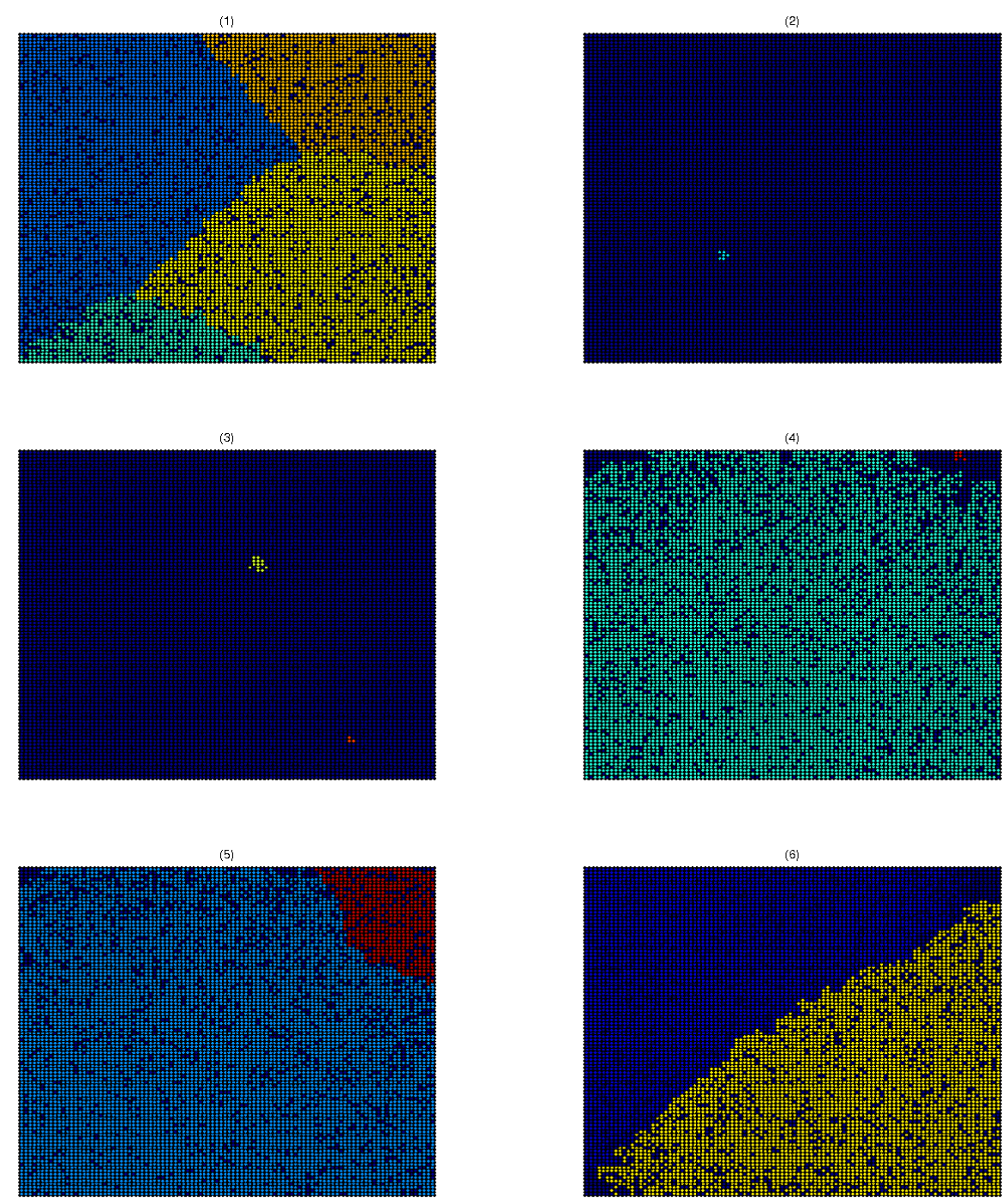

Figure 5.4: Banks evolution in 100x100 society over the course of 100 periods

The overall goal of our framework is to examine the emergence of an interbank 
market as an answer to an observed need in our society. That being said, we start with a society where banks emerge and are subject to bank runs, but nevertheless operate in isolation from one another. In other words, we assumed a world with no interbank market yet.

In such a world, we find that the norm is fast death of banks just a couple of periods after their birth. That need not be surprising, as their initial guess of liquid customers starts as a random variable, and they start with no reserve to protect them from the case of underestimating that ratio. On the other hand, the longer they persist the lower the probability of a bank run, since they accumulate reserves and eventually improve their estimates of early customers through observing the actual world. As a result, whereas most banks fail, the few surviving ones eventually expand to the whole society and achieve a degree of stability that can no longer be destroyed by the individuals liquidity shocks, while at the same time accumulating reserves to further guard their positions.

\subsubsection{Extensions}

Clearly our model generates a banking environment with very specific properties, mainly a few market players, generated after a long episode of bank runs. This bears a close resemblance to nineteenth and early twentieth centuries banking system in the US, an era characterized by panic and bank runs as regular features, as the 1864 National Bank Act, through reducing the barriers to setting up privately owned banks, contributed to a surge in the number of undercapitalized banks.

While the witnessed development of a monopolistic system can be argued to call for a regulatory interference, the more crucial call is a social one to limit the system vulnerability to the frequent runs as stated in Frexias and Santomero [FS02]: "Regulation is the rational response of the government to these new market failures. A lack of response would result in either financial institutions' excessive risk taking or in the growth and development of monopoly power, which is a natural economic outcome of 
such market circumstances". It was only in 1933 that the government did interfere by the introduction of deposit insurance, a regulatory reaction to limit the observed system vulnerability to runs [Fer08].

Such government intervention can be added to our model through simple modifications, such as requiring banks to hold one unit of numeraire at the beginning of their establishment, which can be viewed as a starting cost or entry requirement, representing the capital of the agent establishing a bank. With this simple modification, we found that with the same parameters and rules as before, a significant increase in the number of successfully established banks, from just one or two in most cases to multiple banks. While reserves and other possible regulations seem intriguing to examine, we move in a different direction in the next chapter, allowing banks to seek stability through the possibility of an interbank market. 


\section{Chapter 6}

\section{Interbank Market}

Our banking system so far had no entry barriers and no government intervention, yet we restricted banks from interacting with each other, that is, we imposed no interbank market. As witnessed from the previous chapter, under such an environment banks face the possibility of bank runs together with the draining of their reserves accompanying the misestimation of their depositors liquidity needs. Observing the establishment and growth of banks we concluded that in the long run, and through a long experience with bank failures, banking turns into a monopoly or an epoch with few market players.

This chapter is devoted to equipping banks with their own learning tools and allowing an interbank market, observing its effect of that on our banking system.

We start the chapter by introducing the Allen and Gale model [AG98], which, building on the Diamond and Dybvig model, illustrates how and why banks choose to deposit with each other forming an interbank market. Building on their intuition, we explore the possibility of the creation of an interbank market within our society. We equip our banks with tools to learn from their environment and experiences and to build trust in their estimates. Based on this we determine under what conditions they choose to interact and how they interact with other banks.

We compare the banking environment when banks are allowed to interact versus 
when they are working in isolation. We further conduct a scenario analysis examining the performance of our model under different parameter values. We then conclude the chapter by examining our model under a different and more evolved society structure, where some individuals in the society face correlated preference shocks.

\subsection{The Allen and Gale Model}

As in the Diamond and Dybvig model, there are three times $t=0,1,2$ and a single consumption good acting as a numeraire. The consumption good can be invested in two assets, liquid and illiquid. One unit of consumption good invested in the liquid asset at time $t$ yields one unit of itself at time $t+1$, that is the liquid asset represents a storage technology. On the other hand, one unit invested in the illiquid asset at time $t=0$ yields $R>1$ units at $t=2$, but only $r<1$ units if the investment is interrupted at $t=1$. The economy is divided into 4 identical regions labeled $A, B, C, D$, each corresponding to a single bank (or a representative bank).

Consider a society consisting of a continuum of ex-ante identical customers, each with one unit of the consumption good at $t=0$. As in Diamond and Dybvig [DD00], each individual is assumed to be an early (impatient) consumer with probability $\omega$ (i.e. wants to consume at time $t=1$ ) or a late (patient) consumer with probability $(1-\omega)$ (i.e. wants to consume at time $t=2)$. As such, individual preferences are given by a utility function of the form

$$
U\left(c_{1}, c_{2}\right)=\left\{\begin{array}{l}
u\left(c_{1}\right) \quad \text { with probability } \omega \\
u\left(c_{2}\right) \quad \text { with probability }(1-\omega)
\end{array}\right.
$$

where $c_{t}$ is the consumption at time $t$.

The probability $\omega$ varies from one region to another and can take one of two random values, $\omega_{H}$ indicating high probability of being an early customer and $\omega_{L}$ indicating a low probability, satisfying $0<\omega_{L}<\omega_{H}<1$. The realization of this 
random variable for each region depends on two equally likely states of nature $S_{1}$ and $S_{2}$ and is distributed across regions according to the following table:

Table 6.1: Regional liquidity shocks

\begin{tabular}{ccccc} 
& $A$ & $B$ & $C$ & $D$ \\
\cline { 2 - 5 }$S_{1}$ & $w_{H}$ & $w_{L}$ & $w_{H}$ & $w_{L}$ \\
$S_{2}$ & $w_{L}$ & $w_{H}$ & $w_{L}$ & $w_{H}$ \\
\hline
\end{tabular}

Under this configuration, there is no overall shortage of liquidity across society, since whenever two regions experience higher liquidity demand, two other regions experience exactly the opposite situation, but liquidity is badly distributed. The problem posed in Allen and Gale [AG98] is how to maximize the utility of clients in this case by allocating deposits between banks in different regions.

Their solution starts from a centralized perspective, where they characterize the optimal risk sharing between the four regions as a solution to a planning problem. All consumers are ex-ante identical, as such treated as independent of the region and state of nature. In addition, since there is no aggregate uncertainty in the economy, a central planner can choose a portfolio $(x, y) \geq 0$, such that $x+y \leq 1$, where $x$ and $y$ are the per capita amounts invested in the liquid and illiquid asset respectively, as if the bad distribution of liquidity did not exist. That is, given that the planner can transfer resources among different regions corresponding to the state of nature and the realized liquidity shock, the best allocation can be obtained as

$$
\begin{array}{ll}
\gamma c_{1} & =y \\
(1-\gamma) c_{2} & =R x
\end{array}
$$

where $\gamma=\frac{w_{H}+w_{L}}{2}$ is the fraction of early consumers.

Having obtained the first best allocation, they consider decentralizing it in a perfectly competitive banking environment. Assume that only banks can invest in the illiquid asset. Each bank invests in a portfolio $\left(x^{i}, y^{i}\right)$ and offer a consumption plan $\left(c_{1}^{i}, c_{2}^{i}\right)$. Since banks, unlike the planner, can not transfer resources around, they face 
the threat that the fraction of early consumers might be above average, thereby forcing them to liquidate the long term asset and causing a loss in value. On the other hand, as liquidity shocks are negatively correlated between regions, banks can build linkages in deposits between themselves. Banks in different regions exchange part of the deposits (interbank deposit market) at initial time $t=0$ anticipating the liquidity shock.

In constructing the interbank market, the authors considered the three network topologies shown in Figure 6.2:

1. Complete connected case: each bank is connected to each of the other three banks. In this case, suppose that each bank deposits the amount $z_{i}=\left(w_{H}-\gamma\right) / 2$ with each of the other three banks.

2. Incomplete connected case: each bank is connected to only one adjacent bank, yet the network is still connected. In this case, consider each bank depositing an amount of $z_{i}=\left(w_{H}-\gamma\right)$ with the other bank.

3. Incomplete disconnected case: each bank is connected to one bank, yet the network is now disconnected. Similarly, consider each bank depositing an amount of $z_{i}=\left(w_{H}-\gamma\right)$ with the other bank.

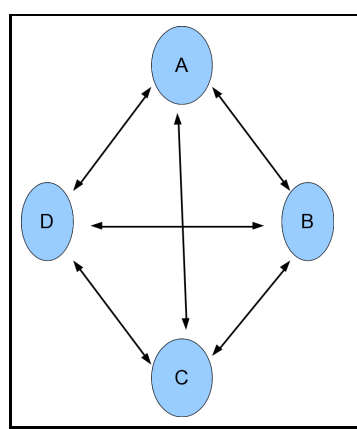

Complete connected

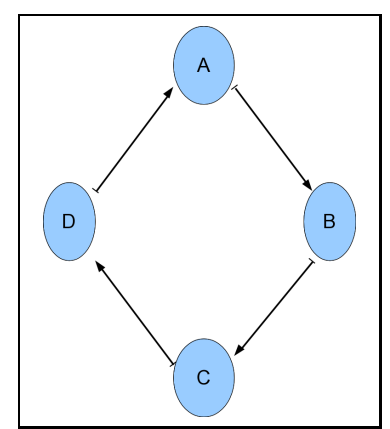

Incomplete connected

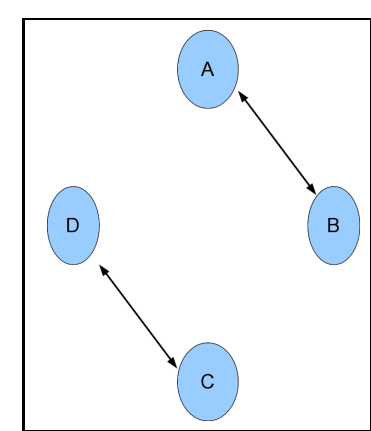

Incomplete disconnected

Table 6.2: Networks topology considered in Allen and Gale

Since the bank deposits indicated above are symmetric, they do not alter the 
bank's allocation in the liquid and illiquid asset at $t=0$. When faced with a liquidity shortage, they follow the "pecking order" of liquidating their liquid asset first, followed by withdrawing interbank deposits, and finally liquidating part of the illiquid asset. Under perfect competition and the symmetry assumed in the model, it can be shown that each network above, with the suggested interbank links, achieves the central planner best allocation with each bank offering the same plan $\left(c_{1}, c_{2}\right)$ and investing according to $(x, y)$ given by $(6.1)$.

Examining the fragility of the networks, it can be shown that if the whole system is to be hit with a liquidity shock $\epsilon$ affecting one of the regions according to Table 6.3, the same interbank linkages that previously safeguarded individual banks can transfer the shock from one bank to another.

Table 6.3: Regional liquidity shocks with perturbation

\begin{tabular}{ccccc} 
& $A$ & $B$ & $C$ & $D$ \\
\cline { 2 - 5 }$S_{1}$ & $w_{H}$ & $w_{L}$ & $w_{H}$ & $w_{L}$ \\
$S_{2}$ & $w_{L}$ & $w_{H}$ & $w_{L}$ & $w_{H}$ \\
$\bar{S}$ & $\gamma+\epsilon$ & $\gamma$ & $\gamma$ & $\gamma$ \\
\hline
\end{tabular}

As bank $A$ is hit, even after withdrawing its interbank deposits from other regions (of total deposited value of $z$ ), the bank is forced to liquidate part of its investment in the long term asset at $t=1$. As such, the amount remaining to pay late customers at $t=2$ is less than their promised value of $c_{2}$. Noting that as the investment payoffs are fixed, and imposing an assumption of perfect information in the economy, late customers will choose to run on their bank at $t=1$ if the bank's remaining payment for them is less than $c_{1}$. It follows that the maximum amount the bank can liquidate from the long term asset without causing a run, called the bank buffer, is

$$
b(w) \equiv r\left(x-(1-w) c_{1} / R\right)
$$

Consider for example the incomplete connected case. If the shock for bank $A$ exceeds its buffer, then this bank defaults and liquidates all of its holdings with 
maximum liquidation value

$$
q_{A}=\frac{y+r x+z c_{1}}{1+z}
$$

Then bank $\mathrm{D}$ will be the first to be affected through a liquidity shock of value

$$
z\left(c_{1}-q_{A}\right)
$$

which has to be raised by liquidating part of its long term assets. If that part is in turn greater than the buffer for bank $D$, then bank $D$ goes bankrupt. What Allen and Gale proved was that, in the incomplete connected case, if bank $A$ went bankrupt and accordingly caused bank $D$ to go bankrupt, then all other banks must also go bankrupt at $t=1$ - a manifestation of contagion causing a system wide crisis. More importantly is the conclusion that there exist parameters values for which any equilibrium in the incomplete connected case involves a system wide crisis, whereas there is an equilibrium in the complete connected case that does not involve such crisis.

The Allen and Gale model elegantly presented a framework for why and how banks choose to form a network that in turn can expose them to systemic risk. Nevertheless, their construction depends heavily on assumptions about symmetry, homogeneity, perfect competition and information, making it difficult to apply it to real banking networks. In what follows we are not aiming at generalizing their model, but rather employ their intuition in discussing why and how banks can interact with each other and form an interbank market in an agent-based framework. 


\subsection{Banks and learning}

Previously, we argued that banks try to improve their estimate of early customers based on past experience through the updating process

$$
W_{k+1}^{i}=\max \left\{W_{k}^{i}+\alpha\left(\overline{W_{k}^{i}}-W_{k}^{i}\right), \frac{1-\frac{c_{2}}{R}}{c_{1}-\frac{c_{2}}{R}}\right\} .
$$

Here we consider the more general case where banks combine this updating process with a learning mechanism to assess the adequacy of the estimate.

We propose that banks build an estimate for the percentage of early customers combined with an estimate of what this percentage ought to be if we believe the sample is a good representative of the society. In what follows we represent a simple model for banks to learn about their customers and show under what conditions that can motivate the establishment of an interbank market.

\subsubsection{Learning from experience}

Similar to individuals in the society, banks have their own targets which they try to achieve by observing their surroundings. For simplicity we mimic for banks the learning process previously used by individuals and assume that banks try to predict the adequacy of their estimate for the proportion of early (impatient) customers.

They determine the adequacy of the estimate according to two basic criteria: statistical accuracy and the current level of bank reserves. The choice of the first criterion reflects the fact that after observing the true percentage of early customers the bank judges its estimate based on its proximity to the true one using the Wilson score confidence interval [Wil27]

$$
\frac{W+\frac{1}{2 n} Z_{1-\frac{\alpha}{2}}^{2} \pm Z_{1-\frac{\alpha}{2}} \sqrt{\frac{W(1-W)}{n}+\frac{Z_{1-\frac{\alpha}{2}}^{2}}{4 n^{2}}}}{1+\frac{1}{n} Z_{1-\frac{\alpha}{2}}^{2}}
$$


for $Z_{1-\frac{\alpha}{2}}$ is the $1-\frac{\alpha}{2}$ percentile of a standard normal distribution, and for our case $W=\overline{W_{k}^{i}}$ and $n=N_{k}^{i}$ is the total number of clients in bank. The second criterion reflects the bank's worries on its available liquidity, specified as maintaining reserves per customer higher than some predetermined threshold $R_{\text {min }}$.

The two criteria combined reflect the fact observed in the previous chapter that the two main determinants of the success of a bank are a close estimate of the actual percentage of early consumers and a sufficiently high level of reserves. We therefore say that a given estimate $W_{k}^{i}$ is adequate if it falls within bounds of the confidence interval in (6.3) and the amount of realized reserves per depositor $\overline{R_{k}^{i}} / N_{k}^{i}$ is above the threshold $R_{\text {min }}$. Otherwise we say that $W_{k}^{i}$ is inadequate.

Banks have a memory of five periods and use the same set of seven homogeneous predictors described in Section 5.2.3, each forecasting that the estimate of early clients for the next period will be either adequate, inadequate, or neutral (i.e neither adequate nor inadequate), corresponding to banks that have just been created and do not have past experience to build their prediction. Similar to the individuals' case, we update the predictors and their strength as times goes by. In establishing the prediction for period $k+1$, banks choose the predictors with the highest strength at the end of period $k$, with a tie-breaking rule favouring adequate and inadequate predictions over neutral ones and setting opposite predictions to neutral.

Accordingly, banks that deem their estimates $W_{k+1}^{i}$ to be adequate for period $k+1$ believe that their allocation will be enough to satisfy their customers need and to maintain a minimum level of reserves. On the other hand, we argue that banks with suspicion about the adequacy of their estimates have a motive to seek each other and establish interbank links to guard against predicted losses. It follows that for interbank links to be established more than one bank should have this kind of suspicion at the same time, in what we will refer to as a "window of opportunity". 


\subsubsection{Windows of opportunities}

Applying the above predictors to our $80 \times 80$ society for the course of 80 periods $(k=$ 80 ), we find the same type of transient behaviour observed in the previous chapter: upon establishment, banks start with neutral predictions, then through learning they experience a phase of oscillation between inadequate and adequate predictions, and finally established a persistent belief in the adequacy of their estimate.

Figure 6.1 illustrates this phenomenon, with yellow representing a neutral prediction, light blue representing a prediction of an inadequate estimate, and dark blue representing a prediction of an adequate estimate. We observe that a number of banks are established during the lifetime of our experiment, with columns representing time and each row represents a bank and its experiences over time. We see that a total number of 17 banks were established (each in a separate row), yet only 5 successful banks persisted untill the end. We observe that the windows of opportunity for an interbank link (that is more than one bank having yellow or light blue cell at the same time step) is less than half of the periods and occurs in the earlier life of banks. As time goes by, the frequency of such opportunities decreases as surviving banks become increasingly confident (dark blue cells) in their estimates. 


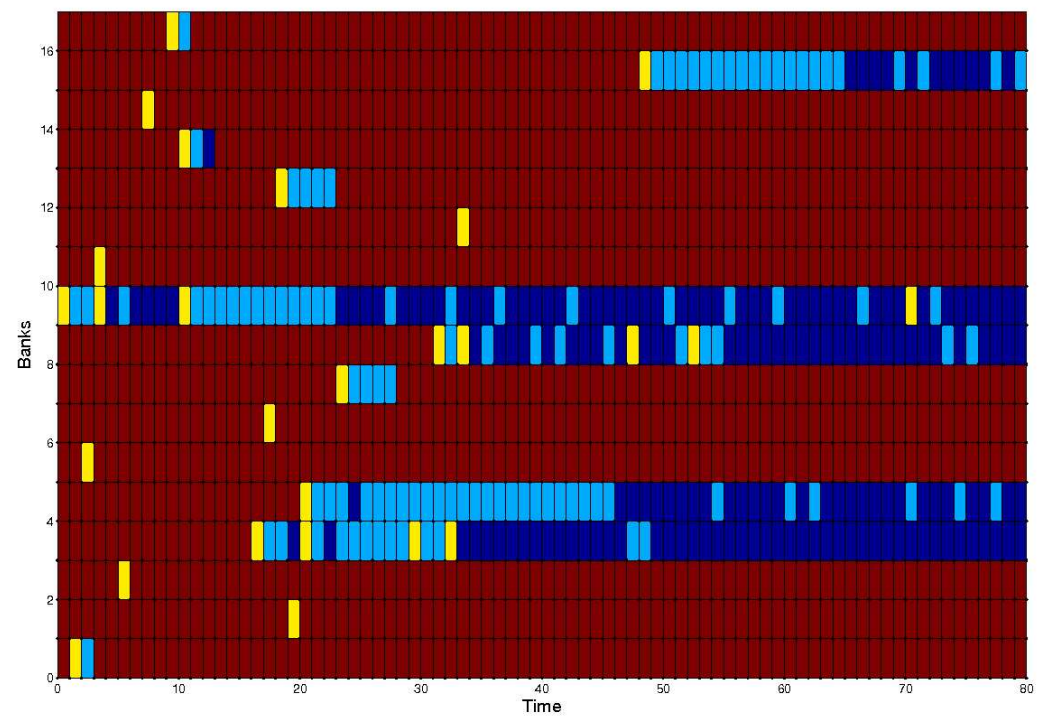

Figure 6.1: Banks learning, an example

Drawing on our earlier discussion, given a high degree of confidence in their estimates, banks will have no motive to establish interbank links. As such, windows of opportunity for interbank links occur in the early periods of banks establishment. Later on we will introduce a mechanism for more dramatic liquidity shocks in the society which can prolong these opportunities when we introduce the idea of "communities" in Section 6.5. Before that, we first describe a way to determine the amount of desired deposits given that two banks want to establish a link with each other.

\subsubsection{Desired deposits}

As indicated above banks having confidence in their estimate for the percentage of early customers rest content that it will yield a good allocation of resources and maintain a minimum level of reserves. On the other hand, banks predicting an inadequate estimate have the incentive to guard against possible losses by engaging in links with other banks. We assume that the interbank deposits yield the same payoffs $\left(c_{1}, c_{2}\right)$ as 
regular deposits.

So far banks have been concerned with their depositors and had no other sources of knowledge. Now we add another layer of banks' knowledge through the assumption that banks also have some global knowledge of the society as a whole. That is, whether the society is in a state of high demand for liquidity, where most individuals are impatient, or in a state of low demand for liquidity, where most are patient. To incorporate this in our model we assume that banks can observe the realized proportion of impatient bank depositors (combined from all banks) from the current period $\bar{W}_{k}$.

When, based on its predictors at period $k$, a bank believes that its estimate $W_{k+1}^{i}$ for the proportion of impatient customers in the next period is inadequate, it compares it with $\bar{W}_{k}$ to determine whether it is overestimating or underestimating the true percentage, leading to two possibilities:

1. Predicted underestimate: if $W_{k+1}^{i}<\bar{W}_{k}$ the bank concludes that it is likely to be underestimating the true proportion of early clients and has an incentive to raise funds by accepting deposits (in-links) from other banks up to the perceived difference of

$$
I_{k+1}^{i}:=N_{k+1}^{i}\left(\bar{W}_{k}-W_{k+1}^{i}\right),
$$

which can be invested in the liquid asset, allowing it to pay the higher than estimated fraction of impatient clients. Instead of accepting interbank deposits, the bank might consider to reallocate its resources and invest more in the liquid asset directly, but at the risk of depleting its reserves in case its original estimate was actually correct (i.e. not an underestimate).

2. Predicted overestimate: if $W_{k+1}^{i}>\bar{W}_{k}$ the bank concludes that it is likely to be overestimating the true proportion of early clients and will seek to deposit 
(out-links) the perceived difference

$$
O_{k+1}^{i}:=N_{k+1}^{i}\left(W_{k+1}^{i}-\bar{W}_{k}\right)
$$

with other banks and withdraw at end of the period $t_{2 k+2}$ if the prediction comes true. Otherwise, if its original estimate was actually correct, then the bank can withdraw its deposits at mid-period receiving $c_{1}$ and as such in both cases satisfy its clients. This is again better than to reallocate resources directly and risk a bank run if the original estimate turns out to be correct.

These two cases are complementary, leading to the emergence of interbank links: banks overestimating are depositors and banks underestimating accept deposits.

\subsection{Building the network}

Given the banks' desire to establish links including the amount and direction of these links (in-links or out-links), the next step is then the establishment of the interbank market or the network.

The task of building the network is not as direct as it appears to be. The first challenge is in how to choose the links. To illustrate, consider the simple example of 4 banks, each of which decided on the amount and direction of its desired interbank deposits: bank $A$ with desired in-links of up to 50 units (accepting deposits from other banks), bank $B$ with desired out-links of 30 units (depositing with other banks), bank $C$ with desired out-links of 40 units and finally bank $D$ with desired in-links of up to 20. Even though we choose a simple example where the combined value of in-links equals that of out-links, indicating all banks' desires in the interbank cooperation can be satisfied, there are essentially an unlimited number of ways to establish the network. It might be tempting to choose randomly any one of the possible combinations to establish the network, nevertheless any structure ultimately determines the stability and performance of the network, and as such needs to be carefully thought or justified. 
The second challenge, as to be expected, is in dissolving these same links when needed. The challenge arises from the fact that the bank liabilities (particularly how much it will pay to its depositors including other banks) depends on the value of its assets, which in turn depends on how much the others banks owe that bank. There is an inherit cyclicity in dissolving such links at the end of the period.

For the first challenge we employee the elegant idea of preferential attachment which is used in explaining the structure of various actual networks discussed in Chapter 2. For the second challenge we introduce a simple algorithm which, as we illustrate, can resolve any kind of cycle.

\subsubsection{Preferential attachment}

For a given period $k$, having a total number of $m:=m(k)$ banks seeking to establish links, denote the $m \times m$ matrix of interbank links by $L_{k}$, with $l_{k}^{i j}$ corresponding to the amount deposited from bank $i$ into bank $j$. Our previous section gives the ideal amounts $O_{k}^{i}$ and $I_{k}^{i}$ each banks would like to deposit with other banks (out-links) and accept from other banks (in-links), respectively. Our task is then to identify how to

determine the entries $l_{k}^{i j}$ to best satisfy these ideal amounts. In other words, how to populate the matrix $L_{k}$ such that the $i$-th row adds up to $O_{k}^{i}$ and the $j$-th column adds up to $I_{k}^{j}$ :

$$
\left[\begin{array}{cccc|c}
l_{k}^{11} & l_{k}^{12} & \cdots & l_{k}^{1 n} & O_{k}^{1} \\
l_{k}^{21} & l_{k}^{22} & \cdots & l_{k}^{2 n} & O_{k}^{2} \\
\vdots & & \ddots & \vdots & \\
l_{k}^{n 1} & l_{k}^{n 2} & \cdots & l_{k}^{n n} & O_{k}^{n} \\
\hline I_{k}^{1} & I_{k}^{2} & \cdots & I_{k}^{n} &
\end{array}\right]
$$

Our algorithm is based on the natural idea that long-established banks are deemed safer and as such are given priority in creating links. The algorithm starts by ordering banks from 1 to $m$ according to their establishment date, from the earliest to the most recently established one, and proceeds by assigning values to each of the links 
according to

$$
l_{k}^{i i}=0
$$

and

$$
l_{k}^{i j}=\min \left(O_{k}^{i}-\sum_{n=1}^{j-1} l_{k}^{i n}, I_{k}^{j}-\sum_{n=1}^{i-1} l_{k}^{n j}\right) .
$$

\subsubsection{Dissolving links}

Now starting at the beginning of the $k$-th period $\left[t_{2 k}, t_{2 k+1}\right]$, suppose that banks establish their interbank links at $t_{2 k}$ according to their predictors and amount of reserves available in their disposal as outlined above in building the matrix $L_{k}$. At time $t_{2 k+1}$, the intermediate period, individuals in the society are hit with the preference shocks, impatient agents seek the liquid payoff, and banks observe their actual percentage of early customers $\overline{W_{k}^{i}}$. At this point, a bank needs to honour withdrawals from both its own impatient clients and from interbank deposits from other banks facing liquidity shortages. We give priority to banks over individual depositors at time $t_{2 k+1}$, so that banks resolve their interbank links before individual customers get their payoff, using netting if multiple banks are to withdraw from each other at the same time. This leads to the following modified version of the scenarios presented in Section 5.4.1:

1. Underestimate $\left(\overline{W_{k}^{i}}>W_{k}^{i}\right)$ : the bank faces an excess liquidity demand. Accordingly, if the bank has any out-links it withdraws all its deposits from other banks (with payoff $c_{1}$ ) to satisfy this liquidity demand. If the bank does not have any out-links, as before, the bank uses its reserves $R_{k}^{i}$, followed by liquidation of part of its investments in the illiquid asset.

2. Overestimate $\left(\overline{W_{k}^{i}} \leq W_{k}^{i}\right)$ : in such case the bank pays any in-links to other banks first (if these banks wanted to withdraw their deposites) and then pays for its own early clients. We can then end up in a case where the interbank links might cause the bank to face an excess liquidity demand even when overestimating the proportion of early clients, pushing it to also liquidate part of its 
own out-links (if any) first, followed by its reserves.

At time $t_{2 k+2}$, the end of the $k$-th period, any remaining interbank links are to be dissolved, with remaining claims of individuals and banks treated equally. As before, a bank faces a bank run if the amount left to its patient depositors (individuals as well as banks) corresponds to a payoff less than $c_{1}$. We see that unlike what happens at $t_{2 k+1}$, the order of dissolving these links between banks does matter. Banks use the payoffs of their own investment in illiquid assets plus any remaining claims in out-links they still have in order to pay their patient depositors and the remaining in-links from other banks. In addition, if that payoff is smaller than $c_{2}$, banks use any reserves they might have to compensate for the difference, as before in the case of no interbank market.

Observe that there is an inherited cyclicity in this kind of payment scheme. The payment one bank will make to other banks (in-links) depends on the amount of money that bank will receive as payoff from its own deposits in other banks (outlinks). For example consider the network below at time $t_{2 k+2}$, where the arrows represent payment flows that should be settled. The amount that bank $A$ will pay back to bank $B$ depends on the amount it will receive from bank $C$, which in turn depends on the amount bank $C$ will receive from bank $B$. 


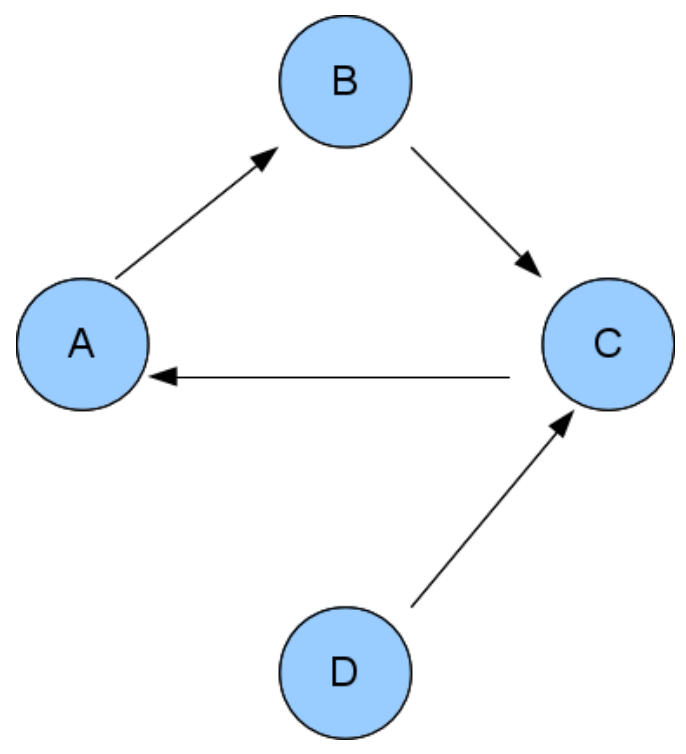

Figure 6.2: Cyclicity in interbank at time $\mathrm{t}=2$, an example

We now introduce an algorithm that can solve any form of payments for networks with this kind of cyclicity based on the following steps:

1. Given a network of outstanding interbank payments at time $t_{2 k+2}$, start solving the edges of the nodes (banks) having only in-links (accepted deposits from other banks), as the amount the bank will pay to other banks can be directly determined since the bank does not have any deposits with other banks and as such does not depend on the asset value of other banks.

2. Next resolve cycles of smallest length by using a netting principle (if multiple cycles exist with the same length we choose randomly between them).

3. Return to first step untill all edges have been dissolved.

Before applying our algorithm, we emphasis the terminology to be used in this section. A bank's outstanding in-links at time $t_{2 k+2}$ represents a flow of payments from this bank to its depositor banks. As such, to ease the discussion of our algorithm, we drawn the networks as arrows of payments due. For example the network in panel 
(1) in Figure (6.2) below shows payments due from banks $D$ and $B$ to bank $C$, from bank $C$ to bank $A$ and from bank $A$ to bank $B$. Applying our algorithm to the this network with the weights indicated in the figures leads to the steps illustrated in the set of panels in Table below. Starting with the network in panel (1) we observe that bank $D$ only has out-payments (toward node $C$ ), as such the amount to be paid from $D$ to $C$ does not depend on any interbank claims and can be solved in step 1 of the algorithm. The edge between $C$ and $D$ is then dissolved (expressed as a dashed line in the network in panel (2)). Now the remaining links form a cycle of length 3 between the nodes $A, B$ and $C$. Following step 2 in the algorithm, we net the exposures on these links as shown in panel (3) by subtracting the smaller exposure from the payments to be made by all banks, leading to no net exposure from bank $A$ to bank $B$. Once we break the cycle, we find that node $B$ only has an out-payment, so we apply step 1 again to dissolve the link between $B$ and $C$ in panel (4). Finally we end up with one remaining link between bank $C$ and $A$, which can again be dissolved by the first step in the algorithm. 


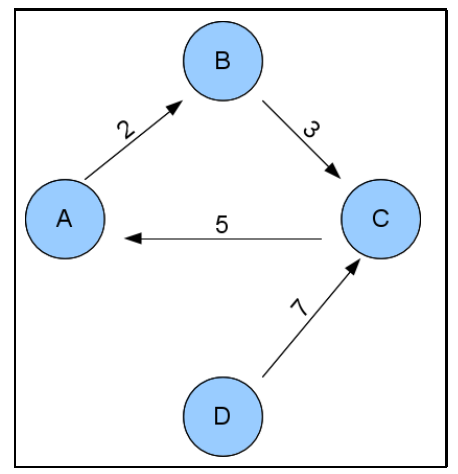

(1)

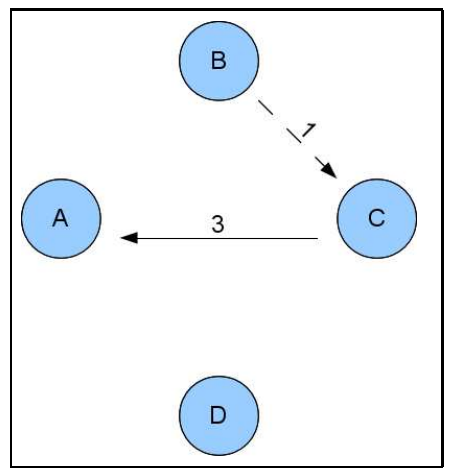

(4)

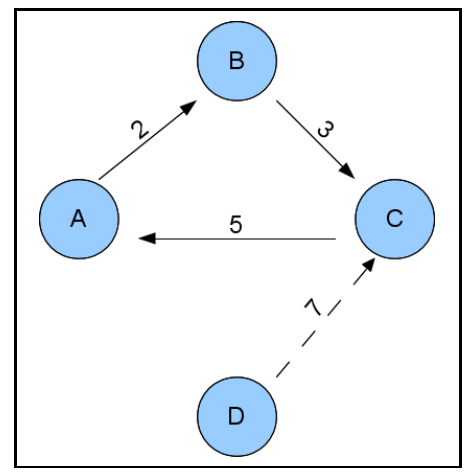

(2)

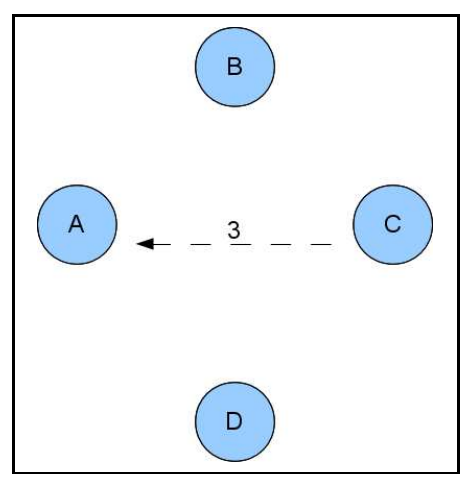

(5)

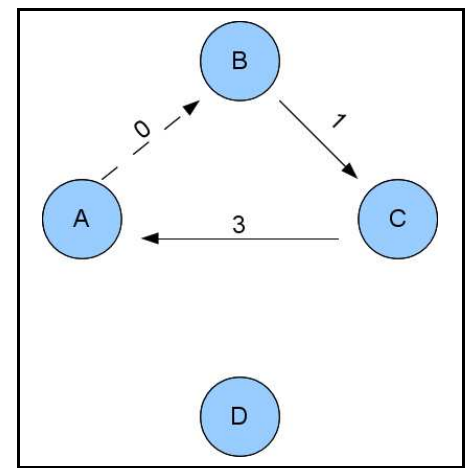

(3)

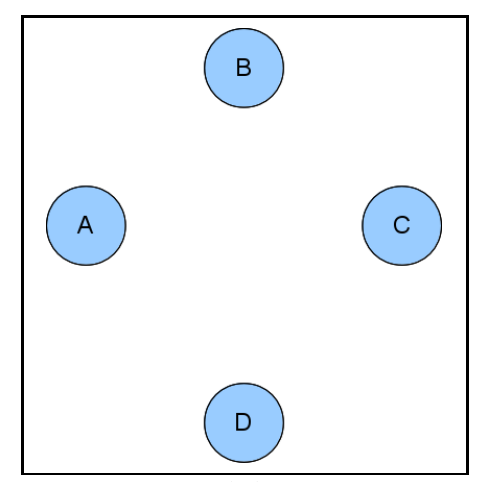

(6)

Table 6.4: Dissolving Cyclicity in the interbank market at time $t_{2 k+2}$ in a network with one cycle.

Our algorithm can be applied to any directed network topology. For a more general example, consider the network in panel (1) of Table 6.5. To start with, there is no node with out-payments only and there are multiple cycles in the network (A-B-G-A, A-B-C-G-A, or A-B-C-D-F-G-A). We proceed with our algorithm until all links are dissolved in panel (8). 
Table 6.5: Dissolving cyclicity in Interbank at time 2, another example

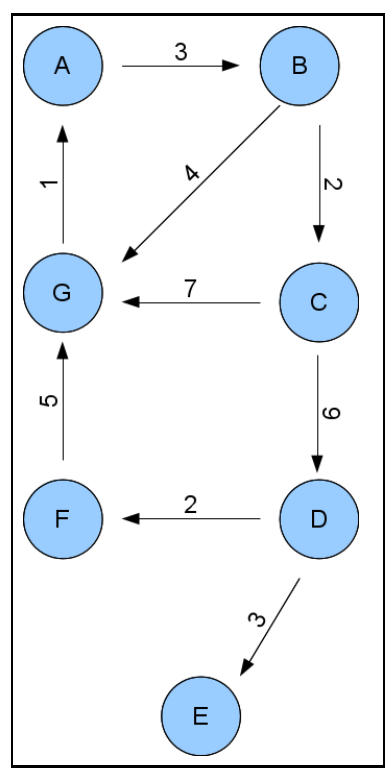

(1)

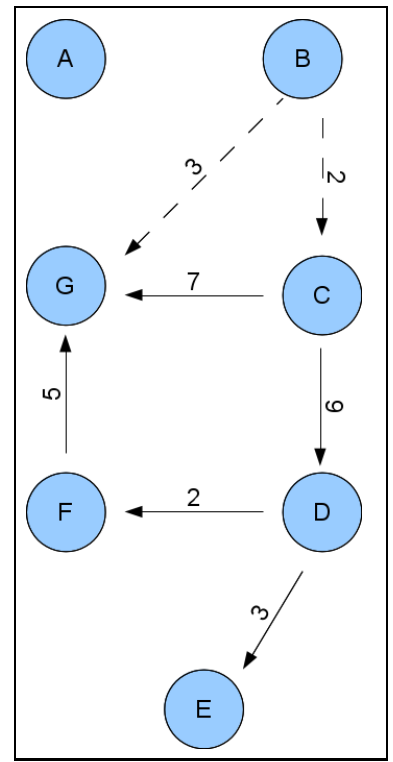

(4)

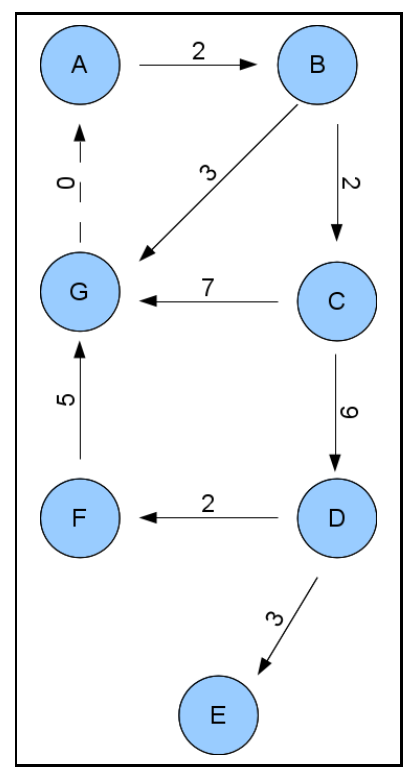

(2)

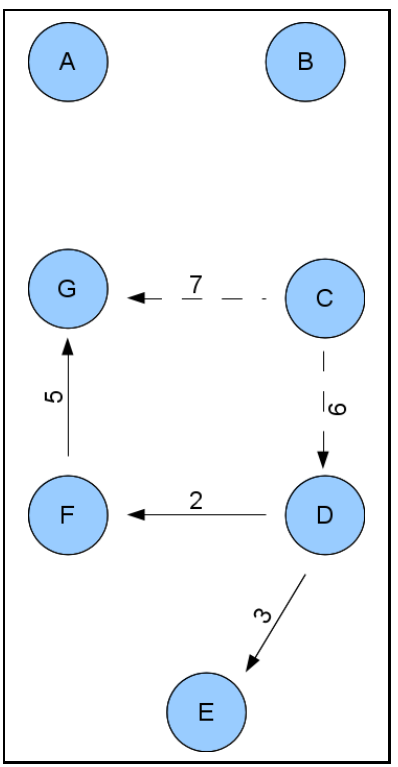

(5)

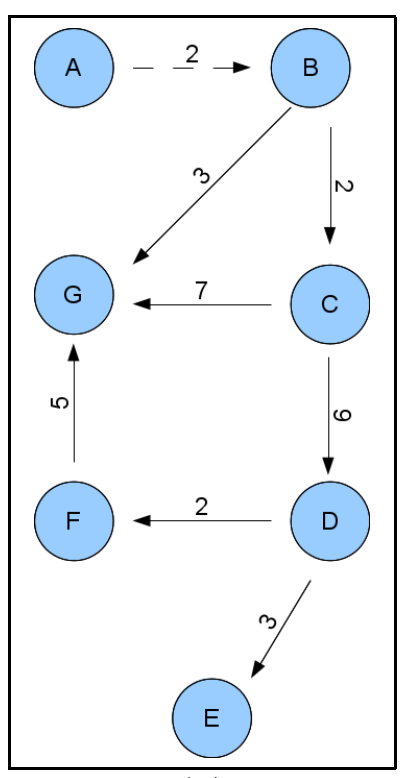

(3)

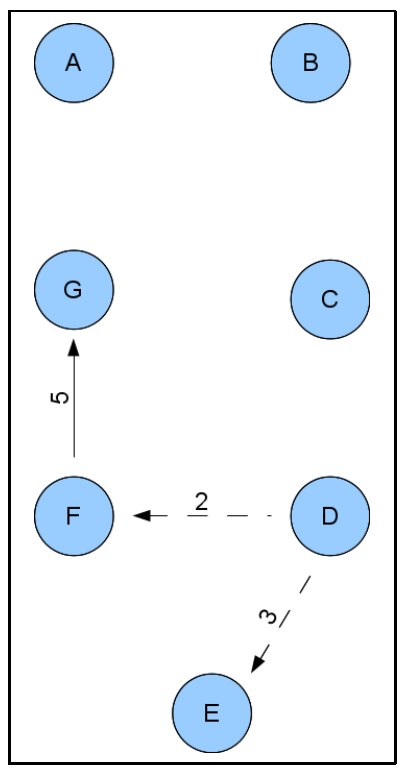

(6) 


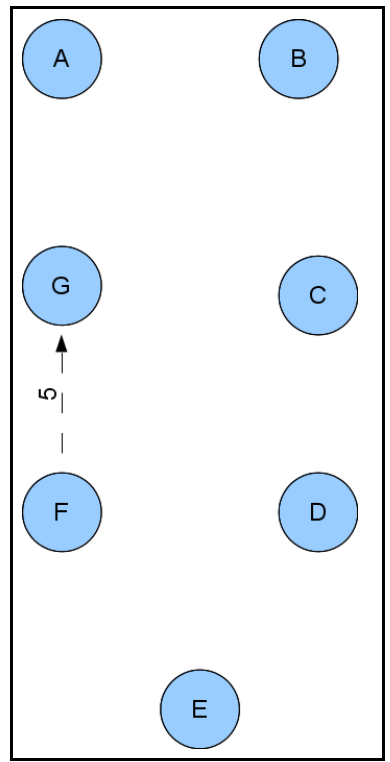

$(7)$

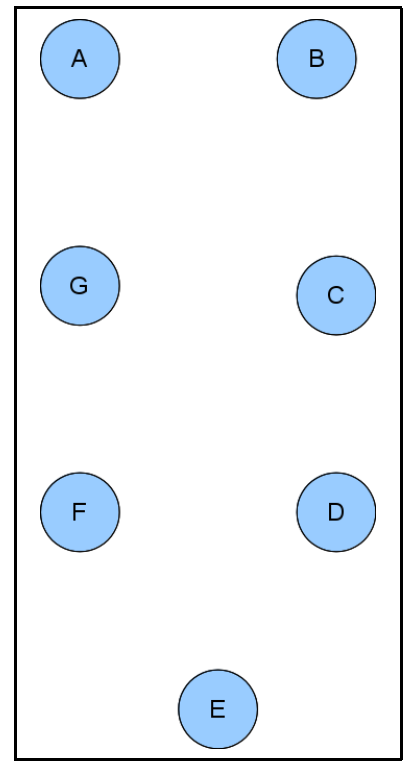

(8)

Table 6.6: Dissolving cyclicity in the interbank market at time $t_{2 k+2}$ in a network with three interconnected cycles.

\subsection{Numerical experiments}

As we observed before, the model without an interbank market resulted in frequent bank failures, few market players and monopoly in some cases. Now as we allow banks to interact with each other the situation changes significantly, and our system appears less prone to failures with more market players than witnessed without an interbank market.

In Figure 6.3 we show four independent simulations of our model where banks were allowed to establish links, with parameters $c_{1}=1.1, c_{2}=1.5, r=0.5, R=2$, $\alpha=0.7$ and $R_{\min }=0.1$. Compared to the cases of no interbank links, we see that more players (banks) are thriving in our society. We see that 2 banks were successfully established out of total 33 banks that tried throughout the society history in the first simulation (top left), 3 out of 18 for the second simulation (top right), 4 out of 17 for the third, and 5 out of 30 for the fourth. 

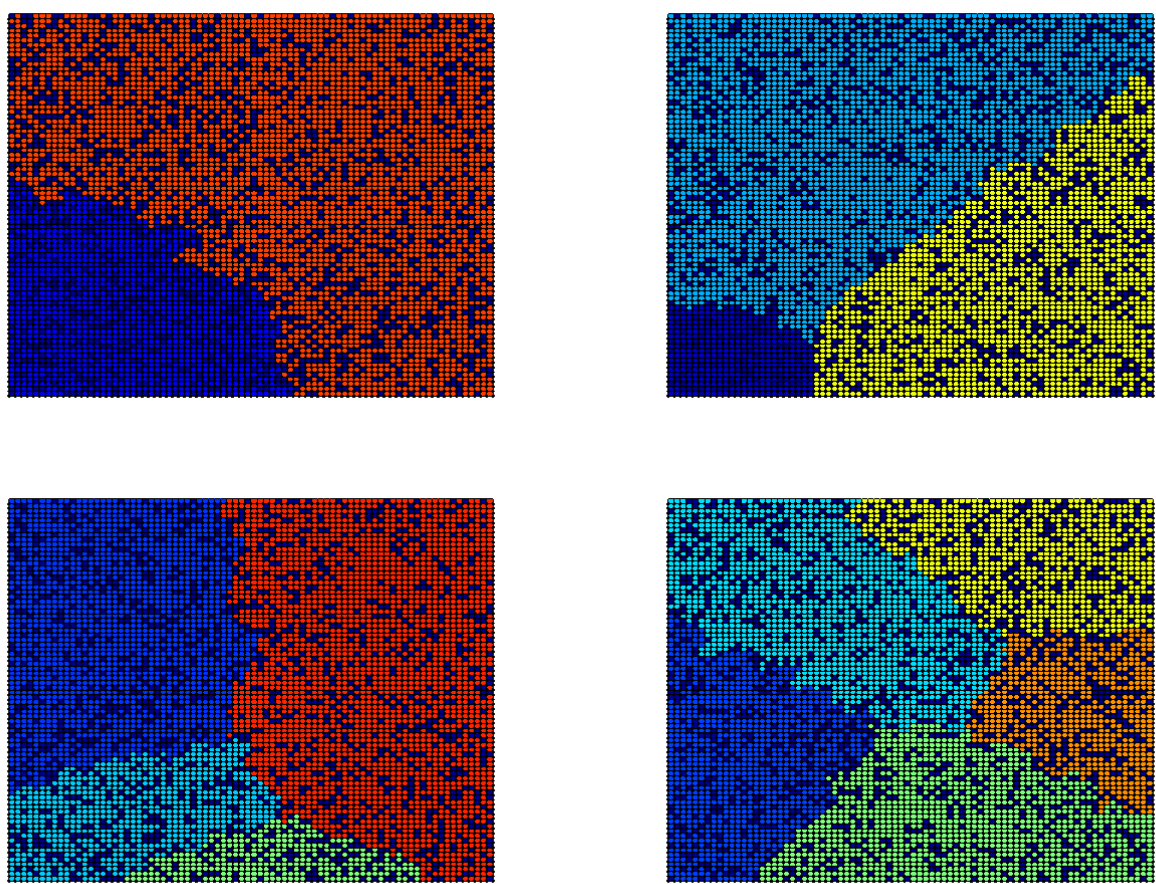

Figure 6.3: Established banks in a $100 \times 100$ society over the course of 100 periods with interbank market

For more conclusive results we implemented 50 independent simulations ${ }^{1}$ of the model (for $80 \times 80$ society over the course of 80 periods) for the two cases with and without interbank links (see Appendix (A.1) for the numerical results). In Figure 6.4 below we report the histograms of the number of banks that survived at the end of the simulation for both cases. As we see there is a clear difference in the distribution of the number of successful banks, with higher frequency of simulations with few market players in the case of no interbank market compared to that where banks were allowed to cooperate. For example, we see fewer monopolies developing in the case of interbank market. On a closer look to the data we find that the number of times when a bank becomes dominant in the society (i.e having more than $90 \%$ of

\footnotetext{
${ }^{1}$ For the numerical comparisons we counted on samples of size 50 independent simulations. While larger sample sizes are to be typically preferable, considering time as well as computational constrains, this particular choice for the sample sizes reflects the rule of thumb in statistical analysis for considering samples of size at least 30 as 'large' samples [MBB99].
} 
total clients) is significantly smaller in the interbank market case ( 3 simulations) than when no interbank market exists (8 simulations).
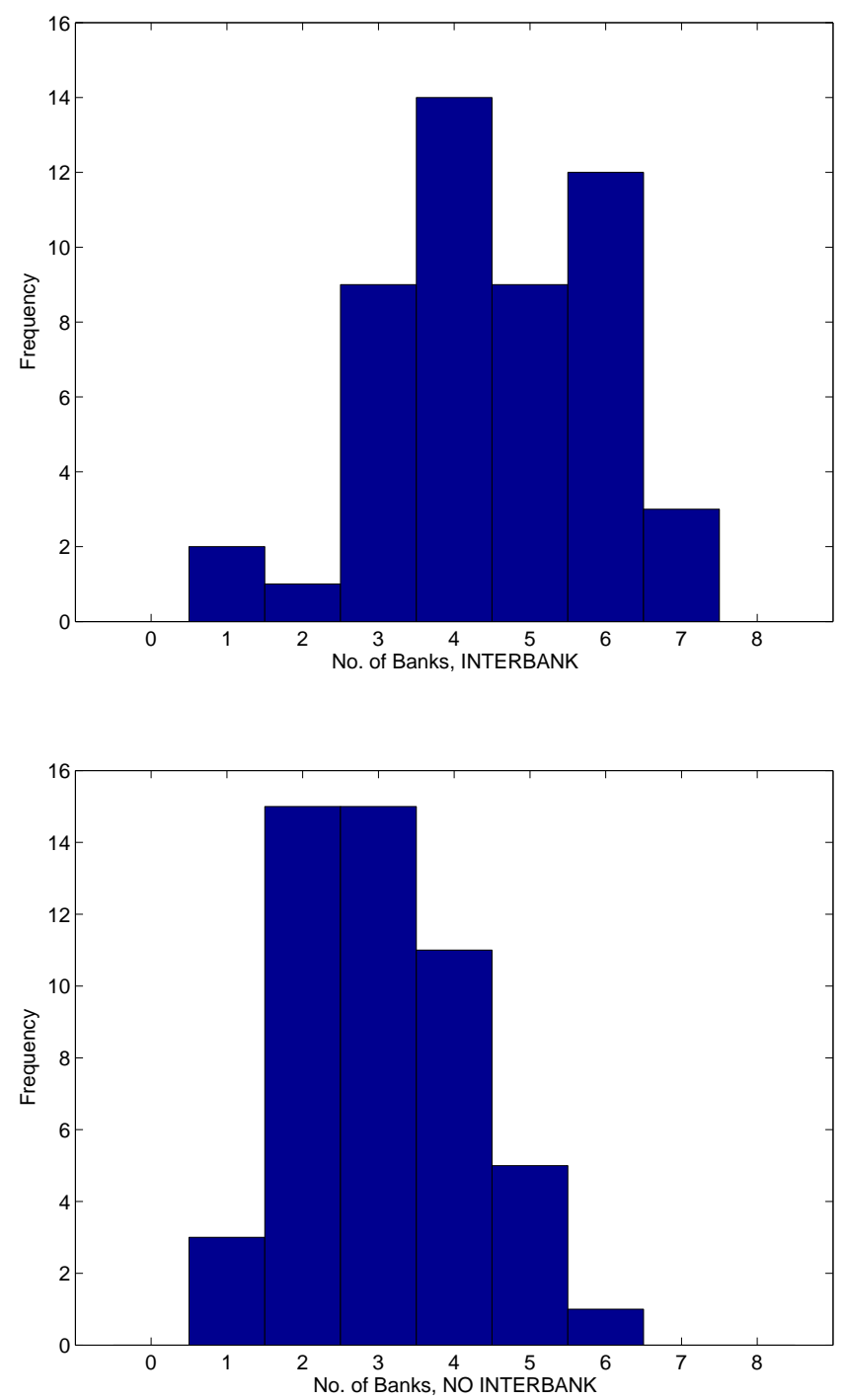

Figure 6.4: Histogram of number of banks established by the end of the 80-th period for the 50 simulations, interbank case (upper graph) and no interbank case (lower graph).

In our inquiry to examine the differences we compared the results along three criteria we deemed to reflect the basic status of the banking in the society: (i) the number of surviving banks relative to the total number of banks that tried to be 
established along the society history, (ii) the size of the largest bank in the society, and (iii) the percentage of individuals who joined a bank by the end of the simulation. As expected there is a significant difference in the banking status under these two cases. Banks were more successful where interbank cooperation were allowed compared to the case where banks were working in isolation from one another. Particularly we found that the average number of banks present at the end of the simulation was 4.5 when banks were allowed to interact compared to an average of 3 when they were not allowed to interact. Taking into consideration the number of banks present at the end of the simulation relative to the total number of banks that tried to establish themselves in the society, we find that when banks were allowed to interact the percentage is significantly ${ }^{2}$ higher than the case where they were not allowed to interact ( $19 \%$ for the interbank case versus $12 \%$ for the no interbank case). On the second criterion we find that the average size of the largest banks where banks are not allowed to interact is $64 \%$ (size measured as number of agents in the bank relative to the total number of agents in all banks combined) which is significantly higher than that witnessed where banks are allowed to interact having the average size of the largest bank as $51 \%$. Interestingly, on the third criterion of bank coverage measured as number of individuals who joined a bank to total society size, the two cases do not record any significant difference (69\% where banks are not allowed to interact versus $73 \%$ for the interbank case).

The results so far suggests that allowing banks to interact by forming interbank links strengthened the banking systems with more market players and less failures. On the other hand it had no effect on the banking coverage in the society which is not surprising given that under the two cases banks offer a fixed payoff contract $\left(c_{1}, c_{2}\right)$, and individuals in deciding whether to join a bank or not do not consider a priori the possibility of bank failures. Before moving to more interesting patterns of individual behaviour in our society, we examine the performance of our model under different

\footnotetext{
${ }^{2}$ We used $90 \%$ significance level for all our comparisons.
} 
parameters.

\subsubsection{Scenario analysis}

In what follows we isolate one parameter at a time to examine its effect on our results holding other parameters at the same level we used before (see Appendix (A.1) for the numerical results). In doing so we analyze results along two dimensions:

- comparison between interbank case versus no interbank case using the new parameter value.

- comparison of the model performance using the new parameter versus the model performance using the original parameter values as reported above (base case), both with and without interbank markets.

\section{Payoff}

So far we used the fixed payoff of $c_{1}=1.1$, and $c_{2}=1.5$. Notice that our individuals are randomly and uniformly distributed between patient and impatient, so that it suffices to change the payoff structure through either $c_{1}$ or $c_{2}$ to see the effect on our results. We run again 50 independent simulations for interbank and no interbank cases changing $c_{2}$.

- Higher payoff $\left(c_{2}=1.6\right)$ : before reporting the results, observe that changing into a higher payoff affects the bank in two direct ways, namely less bank births, as reflected in equation 5.3, and reduced amount of accumulated reserves, so that banks are more susceptible to failures. On the other hands individuals are more willing to join the bank using this new and higher payoff. Comparing the interbank case with the no interbank case, interestingly the advantages that had been enjoyed under the interbank case vanish with the higher payoff. There is no significant difference between the two cases on the first two criteria (the percentages of surviving banks to total banks that tried to establish themselves), 
nor on the size of the largest bank. Not only that, but we find a significantly higher bank coverage in the case where banks were not allowed to interact $(52 \%$ for the no interbank case against $39 \%$ for the interbank case). Such a drop in performance is emphasized comparing the interbank case for the new payoff $\left(c_{1}=1.1, c_{2}=1.6\right)$ with our base case $\left(c_{1}=1.1, c_{2}=1.5\right)$. There is a significant deterioration on the three criteria: the percentage of surviving banks is now $6 \%$ compared to $19 \%$ on the base case, the size of largest bank is $81 \%$ compared to $51 \%$, and finally banks coverage dropping from $73 \%$ to $39 \%$ ). The situation is less dramatic for the case of no interbank market, yet again we observe a significant deterioration on the three criteria compared to the base case: the percentage of surviving banks significantly dropped from $12 \%$ to $6 \%$, size of the largest bank increased from $64 \%$ to $80 \%$, and finally the bank coverage dropped from $69 \%$ to $52 \%$.

- Lower payoff $\left(c_{2}=1.4\right)$ : Lower payoff will affect individuals decision to join the banks (less inclined), yet it is to the advantage of banks in getting established and in accumulating reserves. Comparing the interbank case with no interbank case, banks are thriving under the interbank case which performed significantly better for the three criteria. And again there are significant improvements compared to the base case. For the no interbank case with lower payoff, the percentage of surviving banks significantly increased to $22 \%$, the size of the largest bank significantly dropped to $48 \%$ and bank coverage significantly increased to $75 \%$. As for the interbank case compared to the base case, the lower payoff achieved significant improvements over the first two criteria (percentage of surviving increased to $31 \%$ and the size of largest bank reduced to $39 \%$ ), while on the third criterion the ratio of bank coverage is $76 \%$, yet not significantly different from base case. 


\section{Learning}

Our other comparative parameter is the speed of learning $\alpha$ in equation 6.2, which for the base case was a high value of $\alpha=0.7$. Here we reduce this value and test the performance of our system under a slower learning speed. Unlike the payoff parameter, the effect of the learning can not be anticipated before starting the simulations. While it can be argued that faster learning leads to better estimates, it is to be noted that banks keep acquiring new clients and their initial preferences and random shocks are still hidden to the bank (at least at the beginning).

- Slow learning $(\alpha=0.3)$ : reducing the speed of learning we observe the same pattern as that of the base case. Interbank simulations compared to the no interbank case are performing significantly better on the first two criteria (surviving banks and size of the largest banks) and not significantly different on the third one (percentage of bank coverage). On the other hand, if we compare it to the base case, the results vary. For the no interbank case, there are no significant differences on the first and third criteria compared to the base case, while having a slower speed of learning significantly increased the size of the largest bank from $64 \%$ to $72 \%$. For the interbank market compared to the base case we see mixed results: slower learning performed significantly worse on the first two criteria, decreasing the percentage of survived banks from $19 \%$ in the base case to $14 \%$ and increasing the size of the largest bank from $51 \%$ to $63 \%$, but with no significant difference on the third criterion.

- Moderate learning $(\alpha=0.5)$ : Similar to the slower learning speed, interbank market performs better than no interbank on the first two criteria (16\% for interbank case versus $11 \%$ for no interbank, and $56 \%$ versus $68 \%$ ) and not significantly different on the third criterion. While comparing to the base case we can not find any significant difference in any of the three criteria for the no interbank case. On the other hand, for the interbank case, having moderate learning 
Omneia R.H. Ismail - PhD Thesis - McMaster University - CES

significantly reduced the percentage of surviving banks yet had no significant effect on the other two criteria.

\section{Threshold of reserves}

When banks decide whether to establish interbank links, they consider their estimates coupled with the level of reserves (per client), and having reserves lower than a given threshold $R_{\min }$ raises a warning sign pushing banks to the interbank market. In our simulations we used $R_{\min }=0.1$ for the base case, again we change that parameter while keeping the rest of parameters as in the base case.

- No reserves $R_{\min }=0$ : the effect of reducing reserves has a direct effect in reducing banks' need to interact with each other. In our simulation we notice that reducing the level of reserves to zero worsens the performance of our banks. Compared to the no interbank case (notice that the no interbank case does not depend on the threshold value) the interbank performance is not distinguishable on the second and third criterion yet still significantly higher on the first criterion of the percentage of surviving banks. The worsening of performance is seen when we compare the performance of the interbank market with the base case: the percentage of surviving banks is significantly reduced from $19 \%$ to $15 \%$, the size of the largest bank is significantly increased from $51 \%$ to $58 \%$, but nevertheless there is no significant change on the third criterion of bank coverage (69\% compared to the base case of $73 \%$ ).

- Higher reserves $R_{\min }=0.2$ : increasing the reserves threshold directly affects banks' interactions in increasing their need for interbank links. Again the situation worsens. Although with higher reserves the interbank case outperforms the no interbank case on all three criteria, it performs worse than the base case. Surviving banks decrease from $19 \%$ to $16 \%$, the size of largest bank increased from $51 \%$ to $61 \%$, and bank coverage decreased from $73 \%$ to $67 \%$. Interestingly and crucially, increasing the threshold to $R_{\min }=0.2$ leads to the same effects 
observed when the reserves threshold is $R_{\min }=0$ : there are no significant differences in the performance of the interbank market between the case of no reserves and that of higher reserves on any of the three criteria.

Through varying different parameters one at a time and comparing results between interbank and no interbank cases, we conclude that allowing banks to interact in an interbank market strengthens the system in some instances and in almost all the other cases it performed as well as not allowing them to interact.

Looking at the different results we see that, as expected, banks perform better offering a lower payoff to individuals and interestingly bank coverage in the society does not get worse (it even gets significantly better for the case of no interbank market). On the other hand, offering a higher payoff affects banks dramatically and worsens bank coverage in the society. It is also the one case where allowing the interbank links actually worsens the bank coverage compared to the no interbank case. Interbank markets allow banks to thrive under the favourable conditions of a lower payoff yet does not give them any advantage under the less favourable conditions of a higher payoff.

While having higher learning speed works favourably for banks in some instances, it does not seem to influence the pattern observed for the relative advantage of interbank cases, that is, the interbank market still performs better than the no interbank market on the first two criterion for the different learning speeds implemented. Finally banks performance under the interbank market is sensitive to the level of reserves threshold, both increasing and decreasing the threshold diminished the advantages of interbank market, indicating the existence of an optimal intermediate level for reserve requirements. 


\subsection{Correlated preference shocks}

By structure, our society as presented in our model so far is one where people with different preferences (patient or impatient) are distributed randomly and independently. In this case bank runs happen as a result of misestimation of the ratio of impatient customers. As such, they do not arise as a state of panic where, as described in Calomiris and Gorton [CG91], "bank debt holders at all or many banks in the banking system suddenly demand that banks convert their debt claims into cash to such an extent that banks suspend convertibility of their debt into cash".

Somewhere between the two extremes of independent individuals and state of panics falls a third state where individual depositors seem to share certain a kind of coordinated action for one reason or another. Examples of such coordinated behaviour are the spread of rumours which start with one individual in the society and affects a number of its neighbours causing them to change their preferences to be impatient or the occurrence of location specific shocks such as seasonal demand for cash to satisfy agricultural payment procedures. These and other mechanisms of interaction between individuals lead to the formation of hidden "communities" or "blocks", previously unknown to the banks, where most of the individuals in that community are either patient or impatient customers in a coordinated fashion.

For example we can think of the Allen and Gale model of regions to be a manifestation of this, where the individuals form different communities yet their type (as a group unit of mostly liquid or illiquid concentration) are perfectly correlated with each other. We incorporate this in our model through the introduction of what we refer to as preference regions, or correlated shocks.

\subsubsection{Preference regions}

While the society as a whole has a fraction $p$ of its individuals as impatient customers distributed randomly (see Section 5.2.1), we define a community to be a spatial con- 
centration of individuals with similar investing preferences (patient or impatient). In our examples so far we have used $p=0.5$, in what we referred to as a balanced society, but any other fraction could be used. We establish communities through the following procedure:

1. Number: determine the number of communities to be established as $2 S$, for an integer $S$.

2. Base: randomly select (with equal probability) $2 S$ cells in the society, which will be the base to construct the communities.

3. Reach: set $M$ to be the largest reach for spreading the shock around the base (Chebyshev distance to the base is at most $M$ ). As such the community (or shock) has a maximum reach of a $2 M \times 2 M$ matrix.

4. Size: the size of the spatial concentration is any number less than $(2 M)^{2}$, the maximum total number of individuals in the community reach. For example, taking $3 / 4$ of the total possible size leads to a community size of $3 M^{2}$ individuals

5. Members: for each community base selected, randomly select individuals using its reach unill the community size determined above had been achieved.

6. Construction: finally alter the preferences of all members in a community at once. To keep an overall balanced society, half the number of communities will be established as impatient communities (i.e. a spatial concentration of impatient investors), and the other half will be patient communities.

Figure 6.5 show examples of different communities established in a society of $100 \times 100$ individuals. The first graph (upper) represents two communities with $M=25$, the second graph shows four communities with $M=25^{3}$, and the third

\footnotetext{
${ }^{3}$ Note that according to our algorithm for building the communities there can exist an overlap between the different communities. As illustrated in the second graph, there happens to be an overlap between three communities of patient and impatient agents.
} 
one shows with six communities with $M=15$. For the three examples we chose a community size equals to half of the community reach (size equals $2 M^{2}$ ).
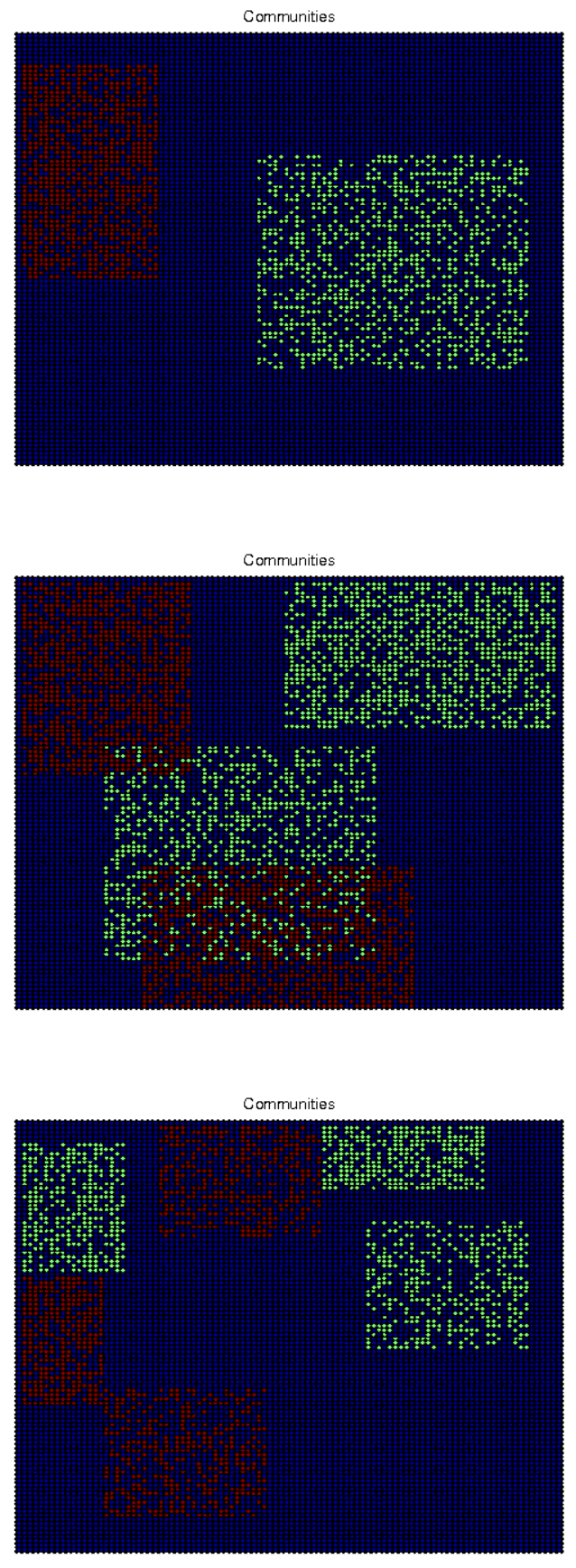

Figure 6.5: Communities established in a $100 \times 100$ society. 


\subsubsection{Numerical experiments}

Now we consider the performance of the banks under the case of shocks in the form of opposite preference regions (see Appendix (A.1). To be noted that every time we impose shocks, they are independent from the previous periods' shocks, as such this is not a community structure, rather a shocks that its place/location can not be anticipated in advance. In the period following the correlated shocks period, individual preferences are set back to their initial preferences, and the rest of the society are subject to their own individuals' shocks.

We start by imposing low frequency shocks, where every 15 periods we construct four preference regions (two patient and two impatient communities). Applying our model, we ran 50 independent simulations for the two cases of interbank and no interbank case (80x80 society over the course of 80 periods), we used parameter selection as that of the base case $\left(c_{1}=1.1, c_{2}=1.5, R=2, r=1, \alpha=0.7, R_{\min }=0.1\right)$. For each of the communities established, we used $M=20$, and community size of $3 M^{2}$.

Under shocks we still witness a model where banks operating with an interbank market outperform that of banks operating in isolation. Banks are more able to survive in the case of interbank market with an average number of survived banks of 3.88 banks against an average of 2.76 for the case of no interbank, (histograms in figure 6.6 ). The average percentage of surviving banks for the interbank case is significantly higher than for the no interbank case ( $18 \%$ versus $10 \%$ ), similarly the size of the largest bank is significantly smaller under the interbank case $(59 \%$ versus $70 \%$ ). As before in the base case (no shocks), the coverage of the banking in the society still reports no significance difference between the two cases $(74 \%$ for interbank case versus $70 \%$ for the no interbank case). On the other hand, comparing the shocks case with the no shocks case, we find that imposing shocks in the interbank case had significantly increased the size of the largest bank compared to the no shocks 
interbank case (increased from 51\% to 59\%), and no other significant differences are witnessed in comparing the shocks with no shocks case.
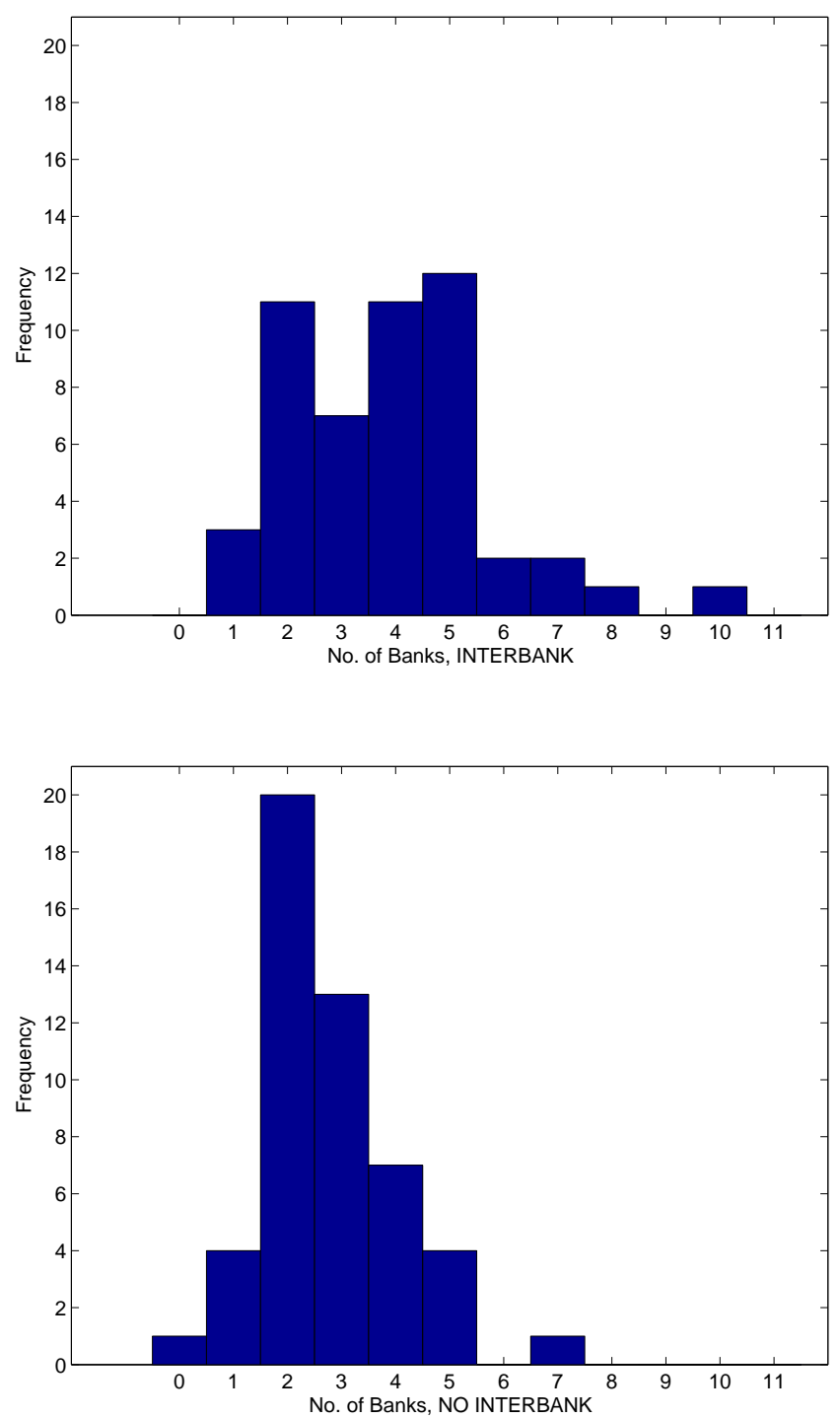

Figure 6.6: Histogram of number of banks established by the end of the 80-th period for the 50 simulations, interbank case (upper graph) and no interbank case (lower graph). Preference shocks every 15 periods.

Now we examine the interbank market in another case of preference shocks. We consider a rather extreme case where we impose the shocks every other period. Under such an extreme case, fewer banks are established with an average of 3 for the 
case of interbank market and 2 when banks are working in isolation, corresponding to the histograms in figure 6.7 .
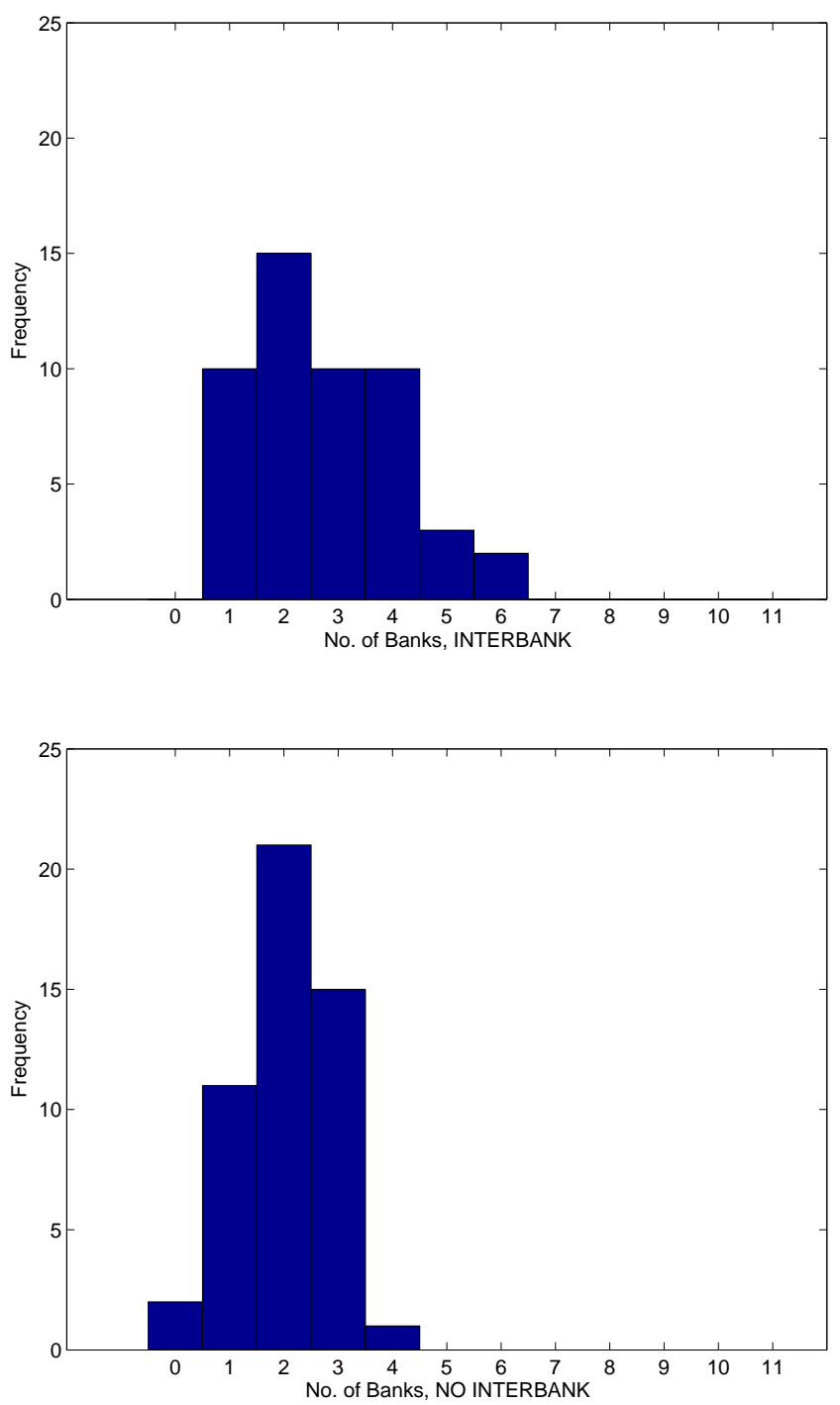

Figure 6.7: Histogram of number of banks established by the end of the 80-th period for the 50 simulations, interbank case (upper graph) and no interbank case (lower graph). Preference shocks every other period.

While comparing the two cases under our three criteria, we find that allowing banks to interact only outperformed on the first criteria while no significant differences were found on the other two criteria. The average percentage of successfully established 
banks had an average of $9 \%$ for the interbank market which is significantly higher than that of the no interbank case (an average of $6 \%$ ). The size of the largest bank under interbank case is $77 \%$ compared to $81 \%$ for the no interbank case (no significant difference). Finally the banking coverage had an average of $67 \%$ for interbank case and $59 \%$ for the no interbank case (no significant difference).

Before concluding our examinations, we consider another special case of preference regions. Instead of imposing periodical liquidity shocks like before we consider the case of established communities. What we called before 'communities' refers to societies where there are spatial blocks of individuals sharing the same liquidity preferences. Unlike our previous test cases where individuals were randomly and uniformly assigned their preferences, we construct societies by imposing four blocks of communities, two blocks of strictly patient individuals and two other blocks of strictly impatient customers. While the society as a whole still has the same fraction of impatient/patient customers now they are spatially concentrated (about half patient half impatient as before). Note that unlike the case of shocks presented before where we temporally change preferences to patient or impatient, having communities means individual preferences are set at the beginning and permanently as part of the community, with initial preferences as strictly patient or impatient.

In such societies individuals in communities who change their preferences will have harder times finding partners. Banks will play a clear role in generating liquidity for the society. On the other hand we find individuals with high liquidity demands ('strictly' impatient customers) will have incentive to join the bank as before, the 'strictly' patient ones will have weaker incentive to join the bank, and banks generated in such spatial communities will have more difficulty keeping alive at the beginning, yet if survived (and unlike the shocks case) it is easier for them to learn about the liquidity demand of their depositors. 
At the beginning of each simulation we constructed fixed communities of $M=$ 20 (maximum Chebyshev distance) of size $3 M^{2}$, and the same parameter values as before. In both cases of banks working in isolation and of the interbank market case, we witness flourishing of the banking system. The average number of successfully established banks is 5 banks in the case of banks working in isolation compared to an average of 6 banks in the case of the interbank market, (the highest average number of banks observed in our simulations) see in figure 6.8. Similarly we find that the average percentage of number of successfully established banks reaches as high as $28 \%$ in the case of interbank market, significantly higher than that when banks are forced to work in isolation (an average percentage of 24\%). Compared to the case of homogeneous society with no communities we find that the average size of the largest bank is reduced to $47 \%$ in the case of no interbank market, insignificantly different from the average percentage of $49 \%$ for the interbank case. Similarly on the third criteria of banking coverage there is no significant difference between the two cases (68\% where banks are not allowed to interact versus $67 \%$ for the interbank case). 

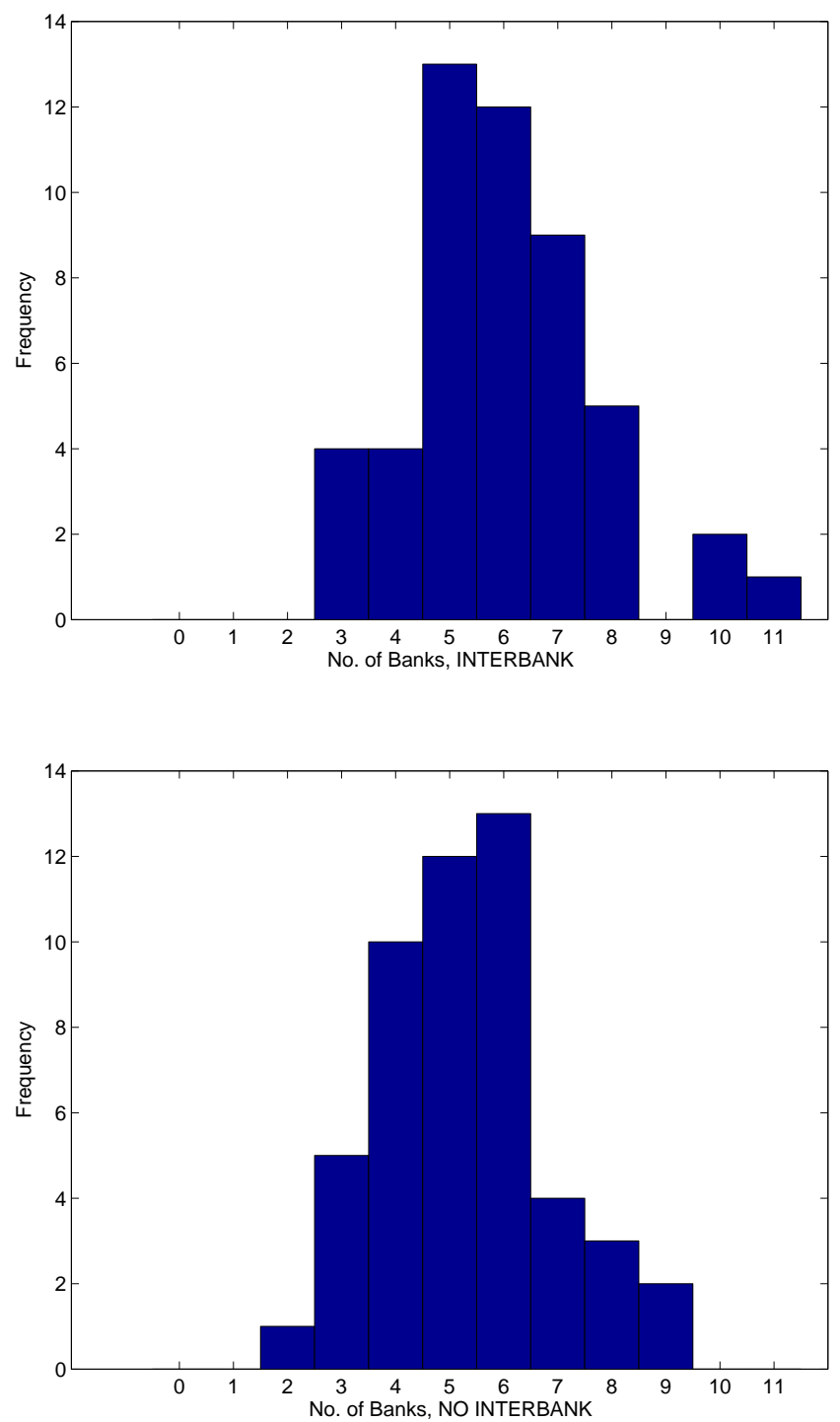

Figure 6.8: Histogram of number of banks established by the end of the 80-th period for the 50 simulations, interbank case (upper graph) and no interbank case (lower graph). Established preference communities.

As demonstrated, the interbank case performed as well as that of the no interbank case in almost all situations, and outperformed the no interbank case in multiple occasions. 


\subsection{Conclusions}

While bank runs and few market players are still witnessed in the banking system when banks are allowed to interact with each other to form an interbank market, there are significantly witnessed improvements compared to the case of banks working in isolation. Allowing banks to interact resulted in fewer failures and a less concentrated banking system.

In addition in a scenario analysis of our parameters, we demonstrated that allowing banks to interact does not worsen/weaken the banking system in almost all cases, and it did improve the performance on multiple occasions. In particular, the interbank market allowed banks to thrive under the more favourable conditions such as a lower payment to depositors, intermediate reserve levels and high speed of banks' learning. Yet allowing banks to interact on less favourable conditions such as imposing higher payments to be paid to depositors did actually worsen the performance of the system.

Finally when considering another more evolved form of society structure where individuals are subject to correlated preference shocks, we found that allowing banks to interact had improved the performance of the banking system when the shocks were less frequent, and did not worsen the performance for the more frequent shocks compared to the case where banks were forced to work in isolation, nor in the case of established preference communities.

Having established that in almost all the cases the banking system under the interbank market is in favour for it compared to the case of banks working in isolation,

next we consider the other side of our story, the effects of the interbank and banking system on the individuals and the economy in what we call 'social measures'. 


\section{Chapter 7}

\section{Banks and Social Measures}

Our work established that allowing banks to interact through interbank deposits yields more stable banking systems with less bank failures and less concentrated market players. While significantly fewer than when banks are working in isolation, bank runs are still common phenomenon in our systems.

There is no doubt that certain measures can be taken to limit such failures, such as deposit insurance, bailouts, or general regulatory policies. Nevertheless it is worth noting that within the simplicity with our model, banks and individuals enjoy a core character of free environment with minimal constraints ${ }^{1}$

Our model has no central planner, and no "obvious" government. By this we mean that while we did not introduce an explicit government agent, we assumed a certain level of organization of the economic activity, like ensuring proper payments for banks depositors, establishing interbank deposits and dissolving these deposits when needed, and other roles. We have what Milton Friedman referred to as the role of the government in a free society [Fri61]: "to determine, arbitrate and enforce the rules of the games". Given this role, citizens and banks work with their limited capabilities to satisfy their needs and desires. As such, stepping into our current setup to handle the "market failures" manifested in few players or frequent runs requires a

\footnotetext{
${ }^{1}$ For example, the contract payoffs $\left(c_{1}, c_{2}\right)$ and the investment payoffs $(1, R)$.
} 
justification in terms of increased "social welfare" or "economic growth". Therefore, any form of intervention is unjustified until we can properly examine the effect of the banking system in our current model on some criterion for social welfare and economic status.

In this chapter we examine such wider effects of banks and interbank markets. We discuss what we refer to as individual satisfaction and show how banks contributed to it. We then discuss the effects of the banking system on individuals' consumption and its inequality. Further we examine the effects of banking and interbank markets on the economic investments and the waste associated with it.

We conclude our examination by considering the banking social and economic effects on these more evolved structures of societies. In particular we consider the scenarios of periodic correlated preference shocks in our society as well as the scenario of permanent communities.

\subsection{Satisfaction}

One interesting consequence of having banks in the society offering contracts with payoffs of $\left(c_{1}, c_{2}\right)$ rather than the individual direct investing with payoff $(1, R)$ is what we argue to be a redistribution of individual satisfaction.

Recall that we started with a society of individuals with equal endowments but different preferences, engaging in investing liquid and illiquid assets and subsequently in a cooperation through voluntary exchange with others to satisfy their own preferences more effectively. Let us refer to the perfect case scenario in which all individuals have satisfied their preferences as the zero optimum, indicating that there is no deviation (zero deviation) between desired preferences and what individuals actually achieve with their investment.

Needless to say such a perfect case is rare, if it ever occurs. Instead, due to individual independent preference shocks, some will wish they had invested differ- 
ently, and out of these individuals some will find trading partners to satisfy their new preferences. For these unfortunates, there is a divergence between their desires and what they got. We call such difference a "deviation", and is measured as the amount actually attained less the amount desired as follows:

\begin{tabular}{|l|c|c||c|}
\hline Case (society with no banks) & Desired & Attained & Deviation \\
\hline Patient individual changed to impatient & & & \\
and could not find partner & 1 & $r$ & $r-1$ \\
\hline $\begin{array}{l}\text { Impatient individual changed to patient } \\
\text { and could not find partner }\end{array}$ & $R$ & 1 & $1-R$ \\
\hline
\end{tabular}

As argued before, such deviation is a form of loss (with the underlying assumption that more is always better) and is a motivation for individuals to join banks.

Now, assume individuals joined banks offering the standard contract $\left(c_{1}, c_{2}\right)$ with $1<c_{1}<c_{2}<R$. Such contracts fundamentally changed the character of our society. With no banks individuals either get what they desired (either did not change preferences or found trading partners) or they suffered a shortage in satisfaction expressed in the negative deviation between desire and attainment. Introducing banks (and ignoring bank failures for now), there is no perfect satisfaction to individual desires, but rather either negative or positive deviation.

\begin{tabular}{|l|l|c||c|}
\hline Case (Individuals in banks) & Desired & Attained & Deviation \\
\hline Patient agent & $R$ & $c_{2}$ & $c_{2}-R$ \\
\hline Impatient agent & 1 & $c_{1}$ & $c_{1}-1$ \\
\hline
\end{tabular}

The realized patient agent (whether initially patient with unchanged preferences or initially impatient with changed preferences) suffers a shortage $\left(c_{2}-R<0\right)$ and is worse off in terms of satisfaction. The realized impatient agent, on the other hand and for the first time in society, obtains a positive deviation $\left(c_{1}-1>0\right)$ in satisfaction. That is, the introduction of banks made some people better than those in the zero optimum, creating a category of "thrilled" (i.e., more than satisfied) individuals. 


\subsubsection{Accumulation and redistribution}

It is interesting to analyze whether banks can create a category of thrilled individuals and hope that the rest of individuals to have on average the same satisfaction as in the no banking case. That is, unlike a zero-sum game, whether we can have some people better off while the rest are unaffected or even better than before. Rather it seems more natural to hypothesize that the rise of such category of thrilled individuals comes at the expense of the rest of society (or maybe the least fortunate category the frustrated ones), indicating a case of redistribution of satisfaction. Answering such question involves first addressing two technical issues: how to view deviations over time and how to assess the differences between individuals in the society.

Since we are studying our society over a span of periods of time, we need a way to aggregate deviations from desire over time. Because deviations are expressed in terms of payoffs $\left(c_{1}, c_{2}, r, R\right)$, we can simply add (accumulate) deviations over time for the individuals.

To elaborate on our idea, consider first a society with no banks, so that individuals engage in direct investing and whenever shocks results in modifying their preferences they search their surroundings for trading partners. At each period we calculated their deviations from desires and accumulate it over the course of 80 periods. Figure 7.1 represents the accumulated deviation (y-axis) for each of the 6400 individuals in our society. As seen in the figure, few individuals achieved the zero optimum situation (what they desired is what they attained) for all the 80 periods, while most of the population deviated from the optimum as they accumulate losses on one or more occasions of changing their preferences while failing to find trading partners. Needless to say those staying close to the accumulated value of zero represents those with strongest preferences (either patient or impatient), accordingly less likely to change preferences with the shocks. 


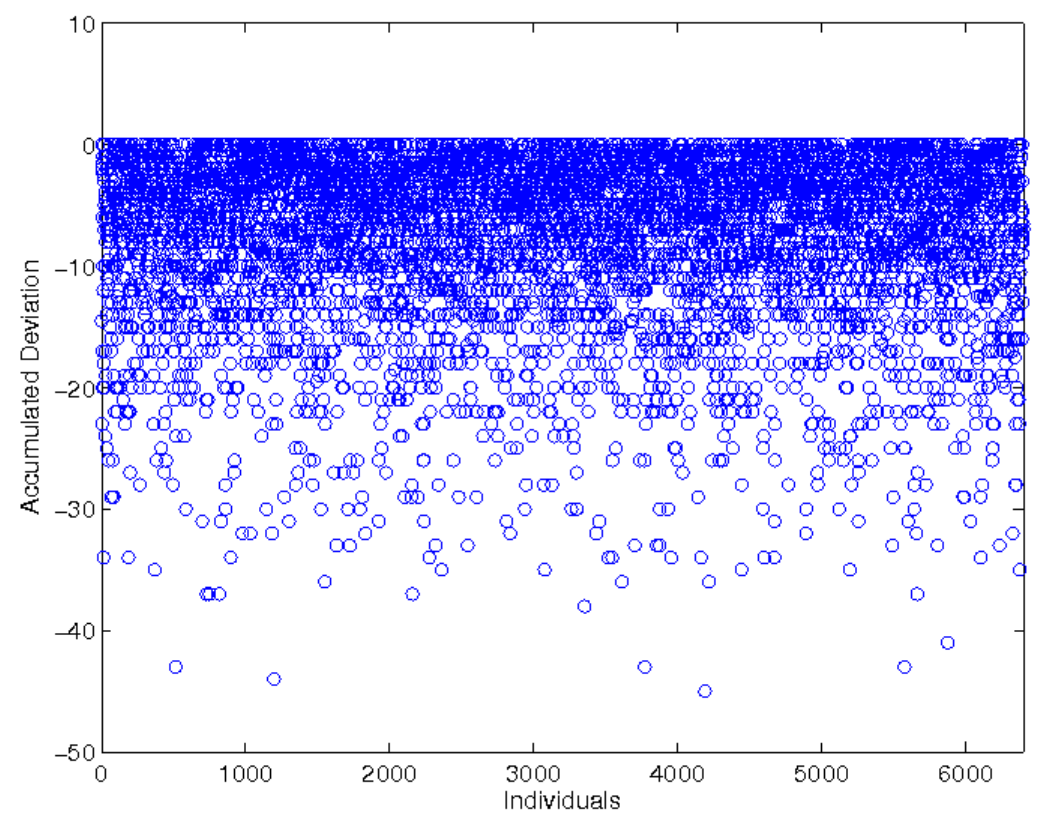

Figure 7.1: Accumulated deviations in a $80 \times 80$ society with no banks by the end of 80 periods.

Now consider our standard case where banks can get established. Measuring the same deviations yields the rather interesting observation that some individuals accumulate deviations above the zero optimum, that is, they successfully obtained positive value at the end of the 80 periods as highlighted in Figures 7.2 and 7.3 for the no interbank and interbank cases. 


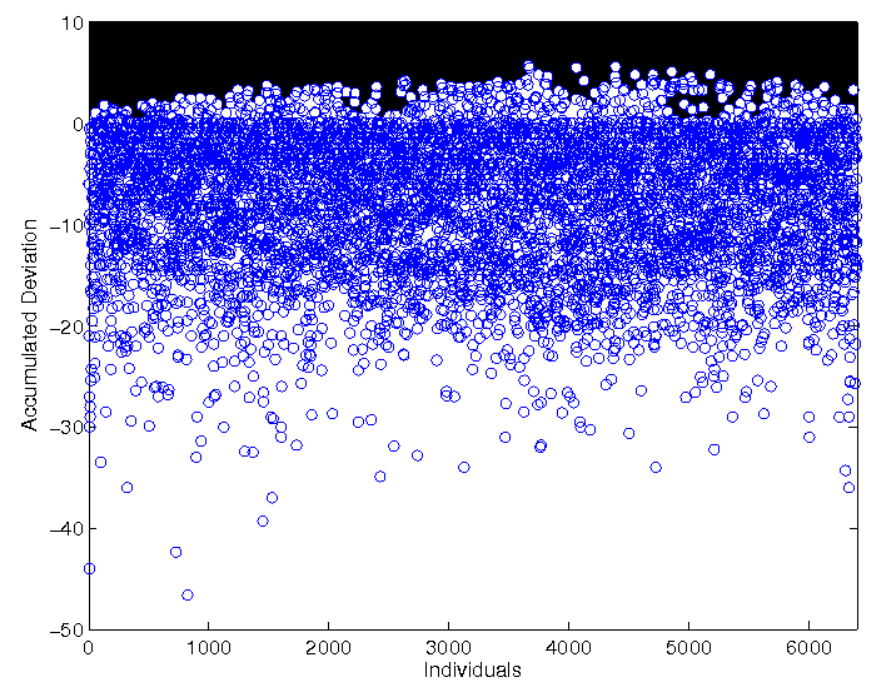

Figure 7.2: Accumulated deviations in $80 \times 80$ society with banks (No interbank market) by the end of 80 periods.

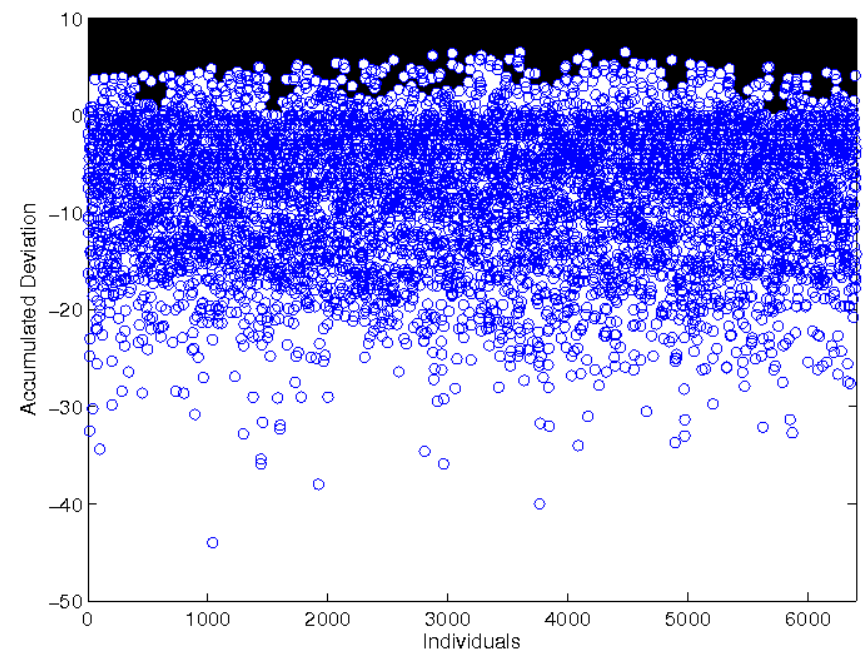

Figure 7.3: Accumulated deviations in $80 \times 80$ society with banks (interbank market) by the end of 80 periods. 


\subsubsection{Differences between individuals}

One of the most common aspects evaluated when measuring differences between individuals is inequality, particularly through the use of the Gini index (or coefficient) [Atk70]. Developed in 1912, this index measures the inequality among values of a frequency distribution and ranges from zero (a case of perfect equality) to one (a case of maximal inequality). It is defined based on the Lorenz curve which represents the proportion of total income cumulatively earned by fractions of the population: a 45 degree line represents perfect equality (e.g., $10 \%$ of the population earns $10 \%$ of the total income, $20 \%$ of the population earns $20 \%$ of the total income, etc). The Gini coefficient is the proportion of the area lying between the line of perfect equality and the Lorenz curve to the total area under the line of equality. For our particular case, we used the Gini index based on discrete distribution as defined by Amartya Sen $([\operatorname{Sen} 76])$ :

$$
G=\frac{N+1}{N}-\frac{2}{N^{2} \mu_{y}} \sum_{i=1}^{N}(N+1-i) y_{i}
$$

for a society of $N$ individuals with incomes $y$ arranged in non-decreasing order $y_{1} \leq$ $y_{2} \leq \ldots \leq y_{N}$ and mean incomes $\mu_{y}$.

As the Gini coefficient is a measure of dispersion, we can use it to evaluate the dispersion in our individuals' deviations from desires. Before applying the index to our data, note that the index can be applied only to nonnegative values of $y_{i}$, thereby requiring a linear transformation for our deviations data. Given the values of our deviations, we measured the satisfaction $y$ of individuals relative to the most frustrated member of society (with the most negative deviation from desires).

As we can evaluate deviations over time through a simple accumulation, we can evaluate the evolution of the Gini index over time as well. Figure 7.4 represents the evolution of the Gini index over the course of 80 periods for the $80 \times 80$ society (using the same parameters as before) for three simulations: one with no banks, one with banks yet no interbank and finally one with interbank market. As it is clear from 
the three curves, there is a substantial rise in inequality in satisfaction (higher Gini index value) over time in the case of banks, while in the case of no banks we observe fluctuations around what it seems to be a mean reverting level over time.

The graphs suggest that the rise of banks is accompanied by the rise of thrilled individuals, accompanied by the rise in satisfaction inequality between individuals in the society.

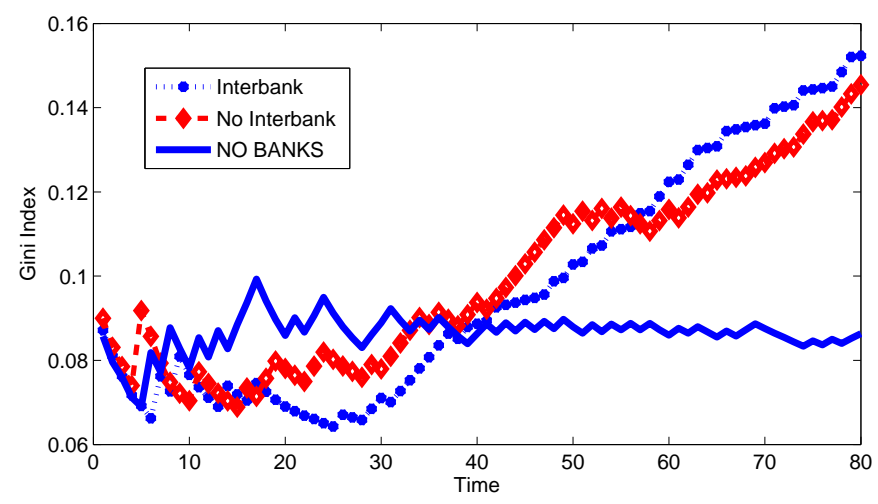

Figure 7.4: Evolution of Gini index over time in a $80 \times 80$ society over the course of 80 periods.

\subsubsection{Numerical experiments}

To explore our initial observation further, we conducted 50 independent simulations as before for each of the two cases of society where banks can be established, the case where interaction between banks is not permitted and the case where interbank market is permitted. In addition we also ran 50 independent simulations where banks were not allowed at all in the society.

Interestingly, we find that our initial observations hold true: introducing banks in a society (whether with interbank allowed or not) substantially increases the Gini index value indicating a case of high (and increasing over time) inequality in satisfaction as compared to societies with no banks. Also as before, in the case of society with no banks the value of the Gini index over time seems to be evolving around a mean value 
as shown in Figures of the results of 50 independent simulation for each case.

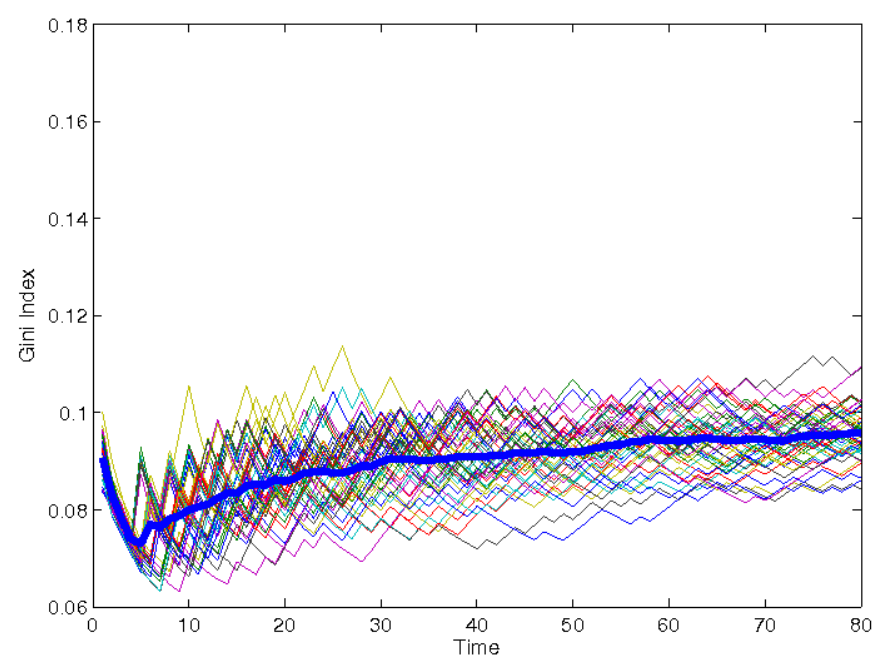

Figure 7.5: Evolution of Gini index over time for societies with no banks - 50 independent simulations of $80 \times 80$ society over the course of 80 periods.

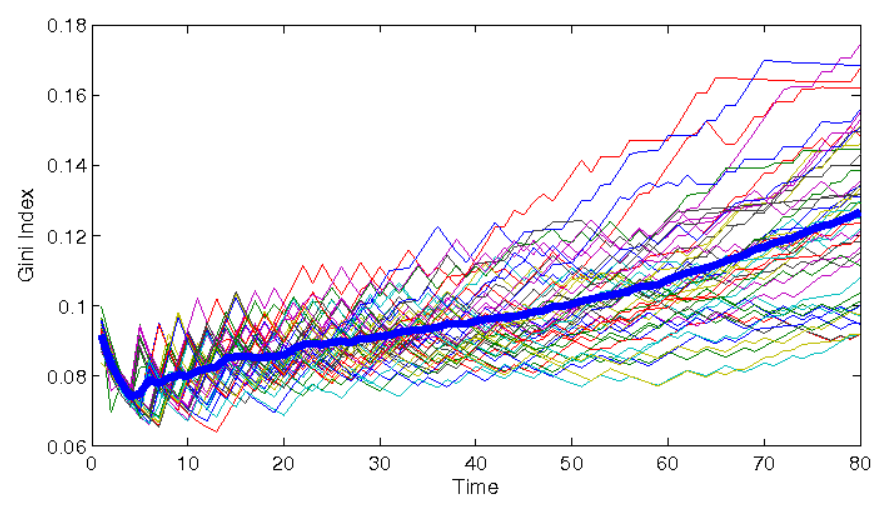

Figure 7.6: Evolution of Gini index over time for societies with banks yet no interbank market -50 independent simulations of $80 \times 80$ society over the course of 80 periods. 


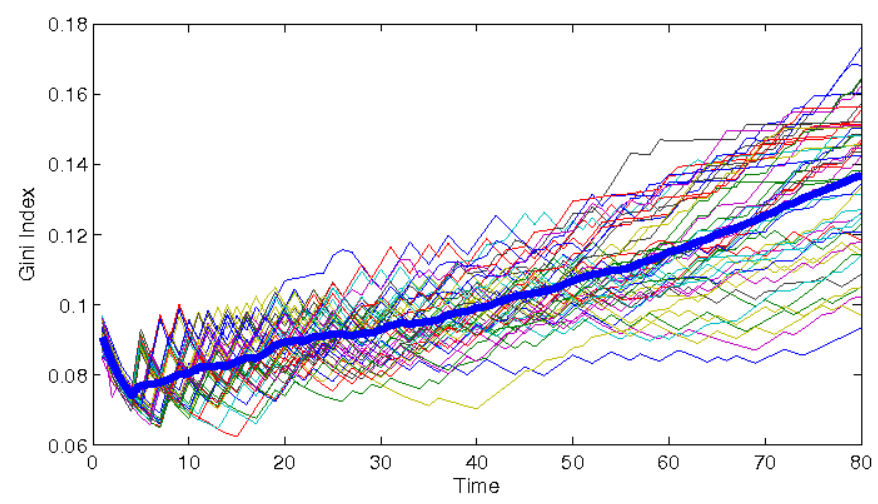

Figure 7.7: Evolution of Gini index over time for societies with banks and interbank market -50 independent simulations of $80 \times 80$ society over the course of 80 periods.

Another hypothesis from our initial results was that the introduction of banks gives rise to a class of thrilled (i.e., more than satisfied) individuals. If our hypothesis holds true, we expect that societies with a higher percentage of thrilled individuals will have a higher Gini index value than societies with a lower percentage. Also societies with higher banking coverage (that is the number individuals in banks as percentage of total individuals) will have a higher percentage of thrilled individuals and higher Gini index.

In a graphical presentation, we compared the values of these measures for the 50 simulation runs for the two cases of no interbank market and interbank market. As observed from Figure 7.8, it does hold true that the rise of banking coverage is accompanied by the rise of Gini index value which in turn is closely associated with the rise of the thrilled class. 

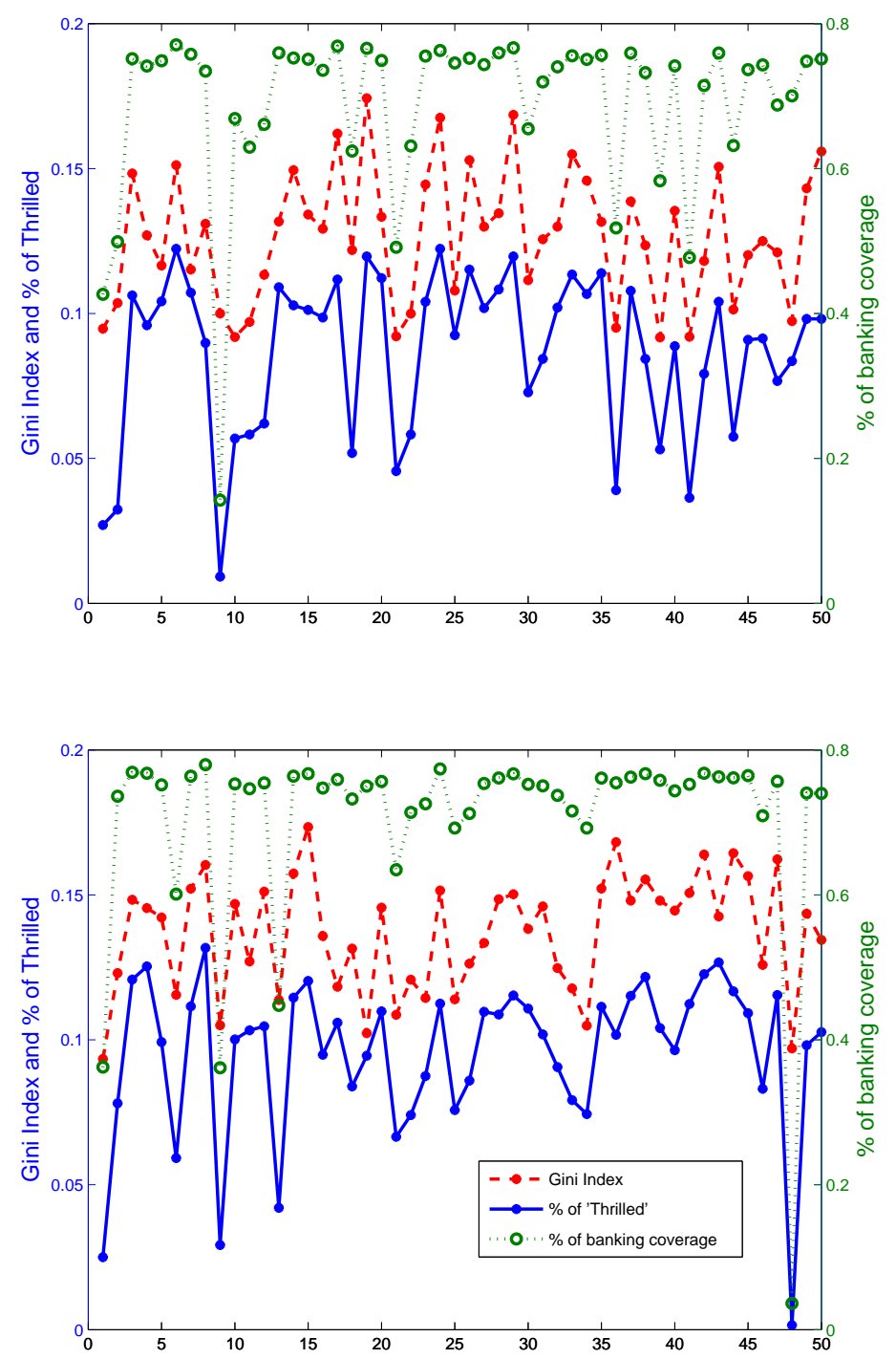

Figure 7.8: Gini index, percentage of thrilled individuals, and banking coverage for the 50 independent simulations of societies with banks with No interbank market (upper), and for societies with banks in interbank market (lower).

Finally, it is interesting to see how the satisfaction of the thrilled compares with the frustration of the less fortunate. Particularly, we can compare the mean of positive deviations from desired with the mean deviation of the rest of the population. If our assumption that the rise of thrilled individuals come at the expense of the rest of 
the society, then we expect that societies with higher mean of accumulated value of satisfaction (positive deviation) to be accompanied by also higher mean of frustration (negative deviation). We see in Figure 7.9 that in both cases of banking systems, the rise of the thrilled is indeed also accompanied by the rise of the frustrated in the society.
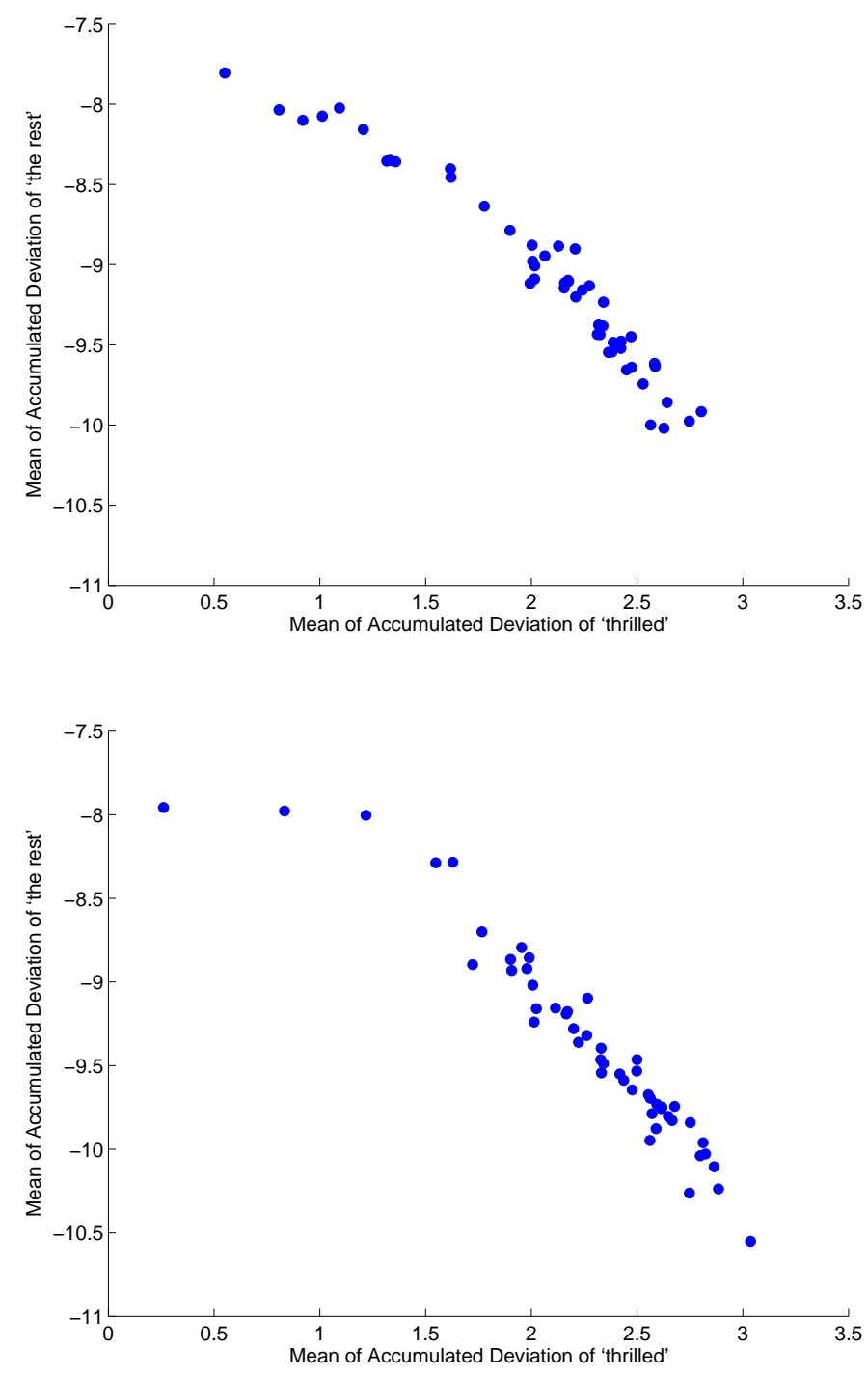

Figure 7.9: Mean of positive and negative deviations for the 50 independent simulations of societies with banks but no interbank market (upper), and societies with banks and interbank market (lower). 
Omneia R.H. Ismail - PhD Thesis - McMaster University - CES

The results we presented so far need not be surprising. It can be seen that banks offering the contract payoff of $\left(c_{1}, c_{2}\right)$ essentially and systematically redistribute satisfaction between the two extremes of the preference scale, favouring the strongly impatient customers at the expense of the strongly patient ones in the long run.

\subsection{Consumption, investment and waste}

In considerable length, we emphasized that banks widened the gap between individuals in their deviation from desires, and in addition and interestingly generated a new class of individuals who gained more satisfaction than possible without banks (we called them "thrilled"). But it is only possible to measure such deviations if we know the true preferences of individuals, which are actually private information and therefore unobservable to us. The only objective way then of measuring the effect of banks on individuals is through an observable quantity like their consumption. If we are to assume that as individuals receive their payoffs (from banks or from their direct investments) they spend them entirely on consumption, we find another interesting observation in this section: banks reduce the gap between individuals measured by consumption, reduce economic waste, but also, unfortunately, reduce the individuals' purchasing power (see Appendix (A.2) for summary of the numerical results for this section).

\subsubsection{Individual consumption, inequality, and the cost of bank- ing}

A key economic indicator is consumer spending volume. For our model, we assume individuals consume their entire payoff at the end of each period, whether it is obtained from depositing in the bank or from their direct investment.

In a simple aggregation process like that of deviations from desire, we calculate the individuals total consumption by adding each individual's consumption over time. 
In our setup of a $80 \times 80$ society of individuals accumulating consumption over the course of 80 periods, the maximum consumption level that can be achieved is that of about 1 million consumption units. This is achieved in the case of every individual investing in the productive technology (the illiquid asset) and holding it to its maturity achieving the maximum possible payoff at the end of each period, corresponding to an accumulated consumption of $80 \times 80 \times 80 \times 2=1,024,000$. However, as our society is structured such that about half of its individuals are patient and half are impatient, we expect the aggregated consumption to be much lower than one million units. For example, in the absence of individual shocks, the accumulated consumption would be of $0.5 \times 80 \times 80 \times 2+0.5 \times 80 \times 80 \times 1=768,000$ instead, and lower than that when some individuals change preferences and cannot find trading partners.

In examining the banking effect on consumption, we find that the aggregate amount spent by individuals in societies with banks is lower than that for the case of the no banks society. To arrive at this conclusion we ran 50 independent simulations for the cases of the no banks society and another of the cases of society with banks (again 50 simulations for no interbank cases and another 50 independent for the interbank case). We found a significant difference between the no bank society case and the cases of societies with banks. In the case of no banks society, the average aggregate consumption level is significantly higher than that when banks do exist in the society $(721,132$ consumption units for no banks society, 716, 708 for society with banks yet no interbank and 716,758 for societies with interbank market). On the other hand, we find that even though the consumption level had been reduced as a result of banks, the differences between individuals have also been reduced as measured by the Gini index. We found that the Gini index for consumer aggregate consumption for societies with no banks is significantly higher than that of society with banks in both cases of no interbank and interbank (13\%, $12 \%$ and $12 \%$ respectively).

Banks were established as separate corporations from individual depositors, who were promised a fixed payoff. Thus, these results should not come as a surprise, in 
their establishment and in their operations banks offered contracts that allowed for a partial transfer of money from depositors to banks themselves in what we call "the cost of banking". Such a transfer is manifested in banks accumulating reserves, and there is little wonder that the aggregate consumption of individuals in our society with banks is significantly lower. Again, and for the same reason, by offering contracts of $\left(c_{1}, c_{2}\right)$ to replace the individual direct investment with payoff $(1, R)$, the gap between individuals under the bank contract is smaller than that of direct investment, resulting in a lower Gini index.

We argue that such a lower consumption level is a direct manifestation of the cost of banking, that is the amount of money that had been directly out of the household pocket found its way into those of bank owners. To examine our claim we compared the consumption level for the case of no banks society with the consumption level of societies with banks, aggregated to the banks reserves. At the end of our 80-period simulations we added the aggregate consumption of individuals with the reserves of the surviving banks to represent the money generated in the society (individuals+banks), corresponding to an average of 721,048 consumption units (out of which 4, 339 units are banks reserves) in the case of banks working in isolation and 721, 527 (out of which 4,768 units are banks reserves) for the case of the interbank market, both of which are comparable to the average consumption of 721,132 for societies with no banks.

\subsubsection{Investments and liquidations}

Another important aspect of our analysis is how introducing banks contribute to the economy as compared to the case of no banks. Looking at investments in the productive technology (that is the illiquid asset) as a measure of economic contribution and the early liquidation of these investments as a "waste" suffered in our economy, we find another interesting feature of banking: societies with banks are not necessarily the ones enjoying the best economic conditions.

Observe again that in our example the maximum amount of initial investments in 
the productive technology is $80 \times 80 \times 80=512,000$. Once again, given that about half of our individuals are patient, we expect the amount of investments in the productive technology to be somewhat less than that.

For societies with no banks, we find that individual's direct investments yielded "successful" investments (that is investments which have not been prematurely liquidated) of an average 224,794 in the productive technology, significantly higher than the aggregate amount of successful investments (combining these of individuals investing directly as well as these investments in the productive technology made by banks) of societies with banks having no interbank market and societies with interbank market (an average investments of 222,524 and 219,966 respectively). That is individuals' direct investments in the case of no banks lead to more resources available for the productive technology in our economy. Two remarks are in order at this point: first, individuals invest based on their known initial preferences, and secondly, we imposed that banks keep their reserves in the liquid asset, rather than reinvesting it in the productive technology.

Nevertheless the individuals successful investments in the productive technology is simultaneously accompanied by more "waste" to the economy in terms of the early liquidation to the productive technology. As a percentage of total investments in the illiquid asset (as well as an absolute value), the amount liquidated is significantly higher than that in the two cases of societies with banks. The percentage liquidated is of $12 \%$ for societies with no banks (a value of 31,326 ) compared to $11 \%$ (a value of 26,952$)$ for societies with banks no interbank case and $12 \%(28,863)$ for societies with interbank market). That is even though our banks yielded an economy with less investments in the productive technology, their investments were more efficient in terms of less waste to the economy as represented by less premature liquidation. 
Omneia R.H. Ismail - PhD Thesis - McMaster University - CES

\subsubsection{Further trends}

In this section we focus on common trends observed in our simulations and how banks introduced changes to the features in society. For societies with no banks, we observe a positive correlation between the total amount invested in the productive technology and the percentage of this amount wasted due to premature liquidation of the productive technology, having a correlation coefficient of 0.4609. Introducing banks into the society and forcing them to work in isolation actually increased the strength of such correlation to 0.7798. Permitting banks to interact in an interbank market reduces the strength of such correlation back to 0.4996 .

Considering individual consumptions and its inequality level, we find that for the case of no banks the aggregate consumption level in the society is strongly positively correlated with the amount of successful investments in the long term asset (coefficient 0.994), as to be expected. The strong relation holds with the introduction of banks as well (0.894 correlation for the no interbank case and 0.874 for the interbank case). On the other hand, we find a negative weak relation between the Gini index and the consumption level in the case of no banks (-0.358). Interestingly, introducing banks alters the relationship between the aggregate individuals' consumption and the Gini index: for societies with banks and no interbank the correlation coefficient is 0.525 and further increased to 0.6554 by allowing banks to interact. Such relationship is further highlighted by considering the aggregate individuals consumption as a percentage of the aggregate consumption combined with banks reserves accumulated at the end. The proportion of the individuals consumption is strongly and positively correlated to the Gini index (0.978 for no interbank case and 0.986 for the interbank case) and seen in Figure 7.10. 

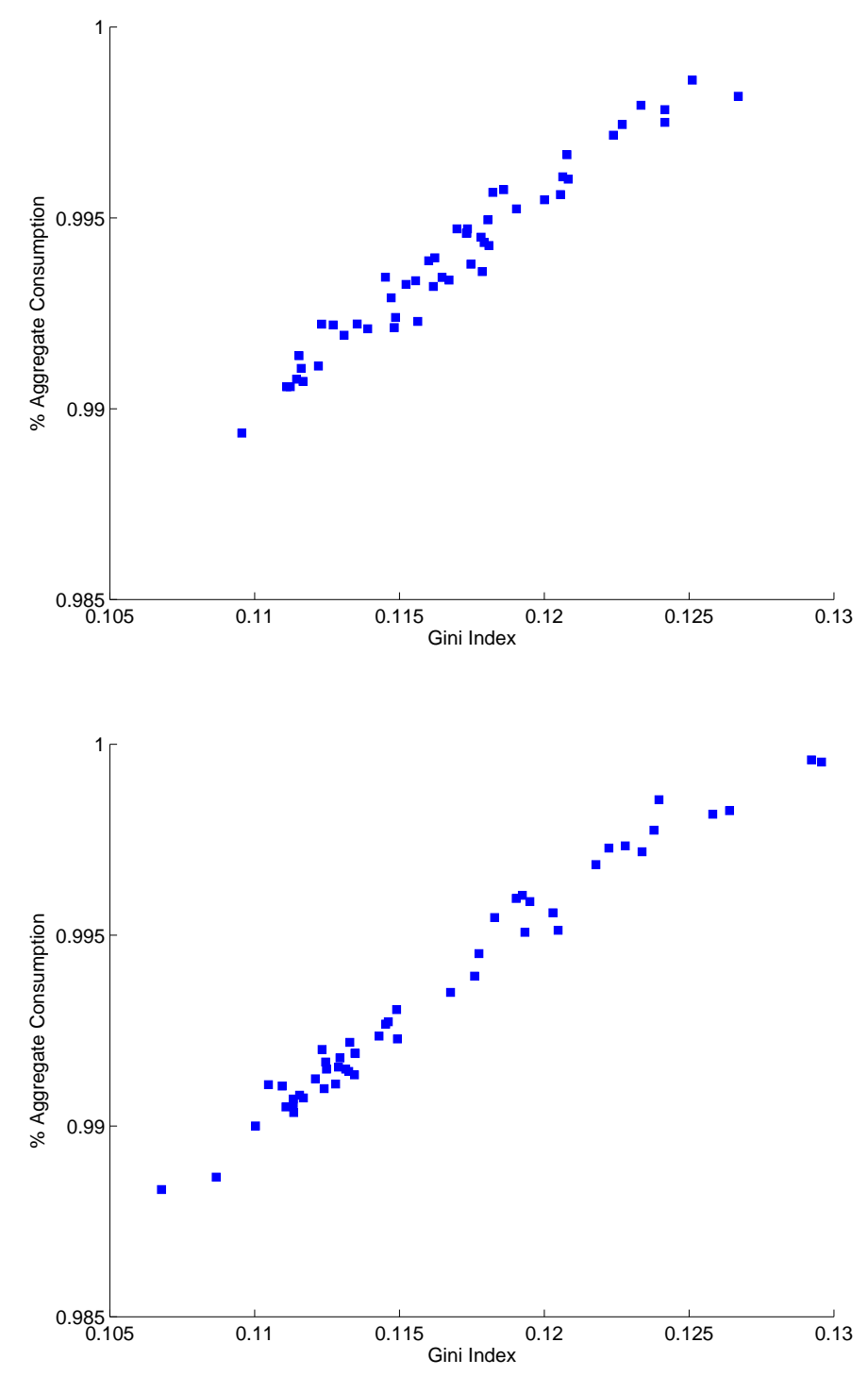

Figure 7.10: Individuals' consumption Gini index and their relative aggregate consumption relative to the sum of the consumption together with banks reserves at the end of the 80th period for the 50 independent simulations of society with banks with No interbank market (upper), and for banks with interbank market (lower).

As indicated before introducing banks redirected the relation between individuals and investments in the economy. As a result the aggregate consumption level is actually negatively related to the percentage of survived banks in the society $(-0.434$ in no interbank case, and -0.518 in the interbank case). Further the inequality level 
decreases with bank coverage and increases with bank success: the Gini index is strongly positively correlated with the percentage of individuals not in banks (0.820 no interbank case and 0.834 with interbank case) as seen in Figure 7.11), and negatively correlated with the percentage of successfully established banks (-0.624 and -0.718 no interbank case and interbank case respectively).
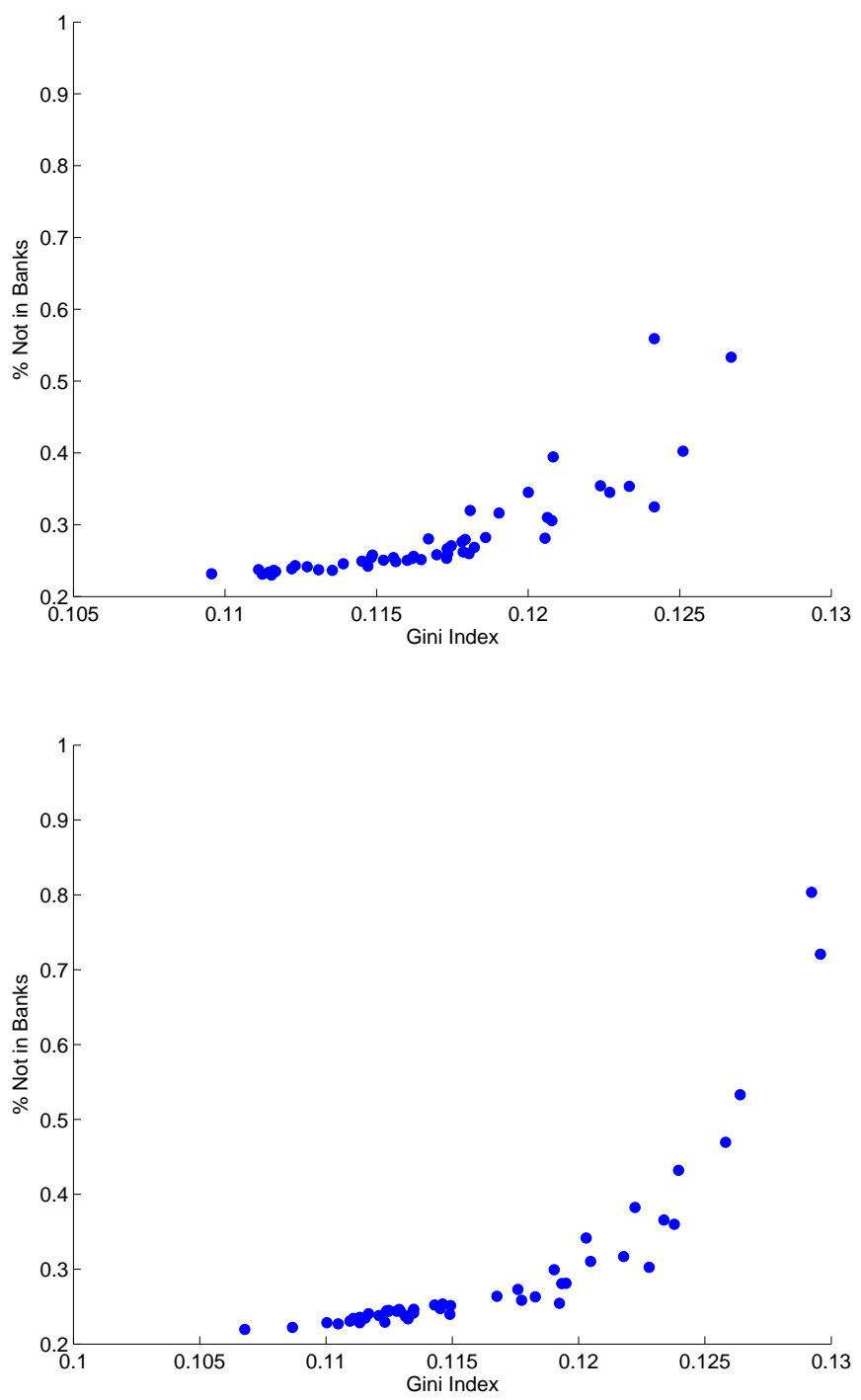

Figure 7.11: Gini index and the percentage of individuals not in banks at the end of the 80th period for the 50 independent simulations of society with banks with No interbank market (upper), and for banks with interbank market (lower). 
Nevertheless the aggregate consumption level as well as the Gini index are strongly negatively correlated to the aggregate banks' wealth measured by the total amount of reserves in the banks' accounts at the end of the 80th period (-0.555 and -0.977 for the no interbank cases, -0.620 and -0.986 for the interbank case, correlation coefficient between the consumption level/banks wealth and the Gini index/banks wealth respectively). As banks prevail in society, inequality decreases between individuals (see Figure 7.12), and aggregate consumption decreases with increases in banks reserves (Figure 7.13). 

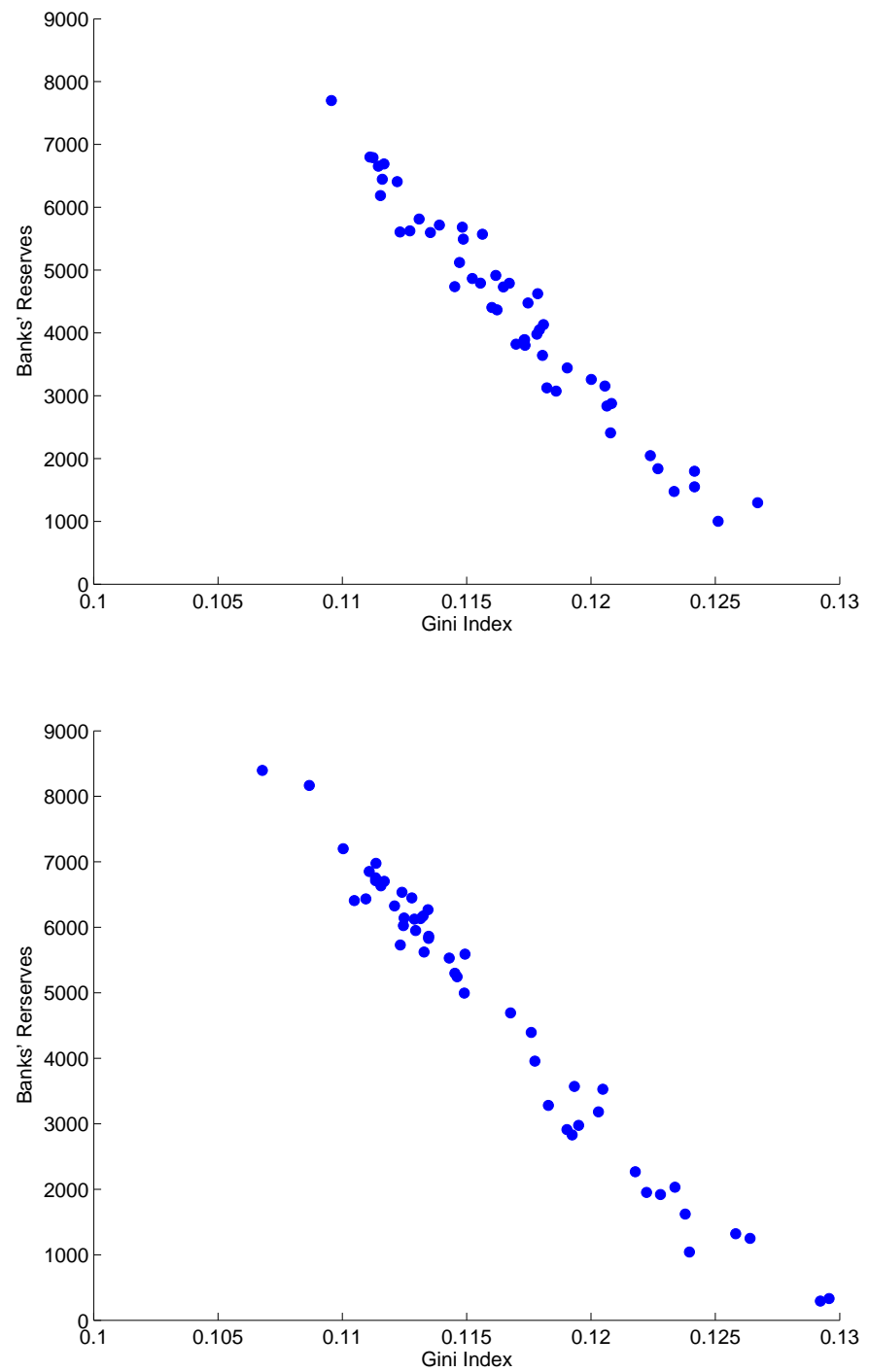

Figure 7.12: Individuals' consumption Gini index and banks aggregate reserves at the end of the 80th period for the 50 independent simulations of society with banks with No interbank market (upper), and for banks with interbank market (lower). 

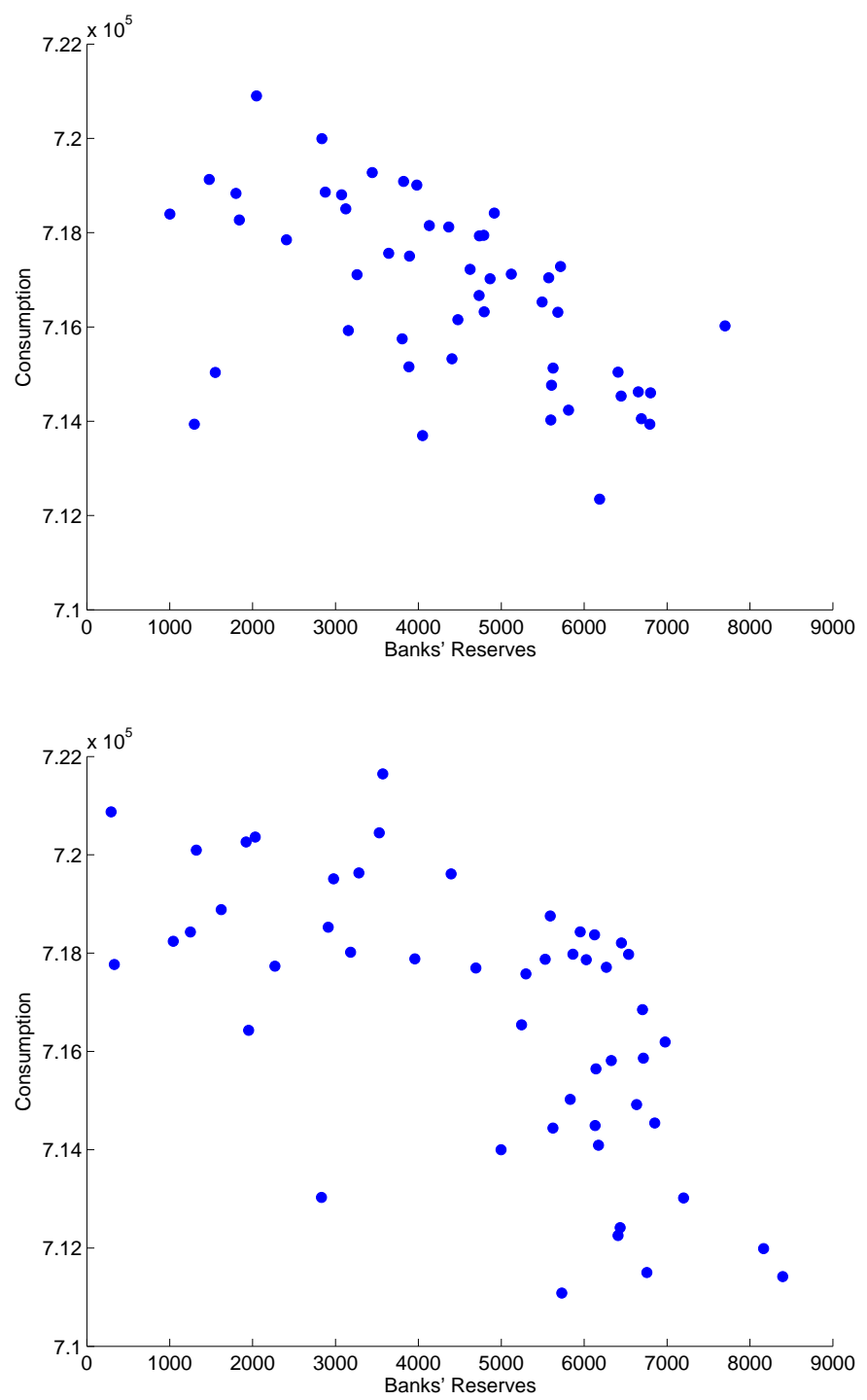

Figure 7.13: Aggregate individuals' consumption and banks aggregate reserves at the end of the 80th period for the 50 independent simulations of society with banks and no interbank market (upper), and for societies with banks in the interbank case (lower)

Finally and as to be expected we find that bank reserves are negatively correlated with the total number of banks trying to be established ( $-0.879,-0.924$ no interbank and interbank case), negatively correlated to the percentage of individuals not in banks (-0.768 and -0.774), positively correlated to the percentage of survived banks $(0.575$, 0.713) and strongly negatively correlated with the amount of prematurely liquidated 
investments in the productive technology in the case of no interbank market $(-0.906)$ and less strongly in the case with the interbank market (-0.584).

Our conclusions should be viewed with the fact that our society is a balanced randomly structured one, there are no communities nor periodic shocks. Such structure implies that with no banks individuals have a good chance of finding trade partners when needed. We expect the results to be significantly different in other occasions where the society has different preference distributions or is less balanced. To examine our hypothesis we ran the above simulations once again (50 independent simulations for each case, for $80 \times 80$ society over the course of 80 periods) for societies with what we referred to before as communities or preference regions.

\subsection{Consumption and investment in societies with correlated preference shocks}

In Section 6.5 we examined the banking performance under the scenarios of periodic correlated shocks to the individual preferences, as well as permanently established preference communities. We established that for the case of periodical preference shocks, allowing banks to interact improved the performance of the banking system when the shocks were less frequent (every 15 periods), and did not worsen the performance for the more frequent shocks (every 2 periods), with similar results holding in the case of permanent communities. In this section we again consider these more involved structures of the society, and examine the effects of banking on the consumption and investment in the productive technology (see Appendix (A.2) for summary of the numerical results for this section).

\subsubsection{Correlated periodic preference shocks}

Imposing a set of periodic shocks essentially affects the banks' capability of surviving by two mechanisms: draining of the bank reserves and confusing the banks learning 
mechanism. As such, unlike before where the loss in individuals' consumption was accounted for in bank reserves in a form of conservation, we expect our systems to suffer uncompensated loss due to these shocks.

As before we start by imposing low frequency shocks, where every 15 periods we construct four preference regions (two high liquidity and two low liquidity). We ran 50 independent simulations for the two cases of interbank and no interbank in addition to the case of no banks, using the same parameters values as before (section 6.5.2).

As expected for societies with no banks, the average aggregate consumption level is significantly higher than that for societies with banks. Particularly, the average aggregate consumption level for the 50 simulated societies is 719,671 compared to 712,803 for the case of societies where banks work in isolation and 712,940 for societies with banking interacting in an interbank market. The uncompensated loss we hypothesized is reflected in the fact that, even accounting for the bank reserves, the individuals' aggregate consumption does not compensate for these reported differences. The aggregate consumption in societies with no banks is still statistically significantly greater than the aggregate consumption for societies with banks even when we add the accumulated bank reserves in these societies (the aggregate accumulated banking reserves is 5,778 for the no interbank case and an average of 6,017 for the interbank case). In addition to the cost of banking, our banking system suffered an additional cost reflected in a pure uncounted for waste due to the shocks.

On the other hand, the Gini index for societies with no banks had an average of 0.128, significantly higher than the case of societies with banks working under the interbank market (Gini index average value of 0.114), yet not significantly different from that of societies with banks working in isolation (average Gini index value of $0.118)$.

As for investment, the amount of successful investment in the productive technology is higher in the case of societies with no banks. The aggregate amount of investments in societies with no banks is 224,116 , significantly higher than that of 
societies with banks, where the aggregate successful investment amount is 220,297 for the no interbank case and 215,915 for the interbank case. Nevertheless, also in societies with no banks we find the highest amount of wasted investments, where the amount of investments in the productive technology that were prematurely liquidated had an average of 32,890 , significantly higher than that of societies where banks were allowed to be established (compared to an average of 27,431 for societies with banks and no interbank case and 31,507 for the interbank case).

Now we examine the performance of our systems under the more extreme case of high frequency shocks. We rerun our simulations (50 independent simulations for each case) under the same parameters values as before, and imposing the shocks every other period. We observe the same patterns with regard to the investments as in the case of the low frequency shocks. The amount successfully invested in the productive technology is significantly higher in the case of societies with no banks (an invested amount of 215,224 ) if compared to the case of societies with banks (an invested amount of 213, 606 for the no interbank case and 199,552 for the interbank case). Such higher successful investments are also, and again as before, combined with higher waste in terms of amounts prematurely liquidated investments in the case of no banks societies is compared to the case of societies with banks working in isolation. The average amount of prematurely liquidated investments in the productive technology is 40,541 compared to 34,497 for the societies with banks yet no interbank market. Interestingly when compared to the interbank case, we find that a rather result where allowing banks to interact significantly increased the amounts prematurely liquidated investments to 44,6330 .

Considering the consumption and banks reserves, increasing the frequency of the shocks to every other period reflects a different interesting case. For the aggregate level of individuals consumption, we find that as before the average aggregate level of individuals consumption is significantly higher in the case of societies with no banks (an average level of 706,953 compared to 701,537 for the no interbank case 
and 699, 024 for the interbank case). Interestingly we find that accounting for bank reserves in societies with banks results in significantly higher amounts if compared to the aggregate consumption for societies with no banks (average level of aggregate bank reserves is 6,820 for societies with banks working in isolation compared to 9,301 for the interbank case). That is in the case of high frequency shocks we find that the shocks dramatically affected the individuals in societies with no banks.

Similarly for the Gini index, the average value in the case of no banks societies is significantly higher than that in the case of societies with banks an average level of 0.1181 compared to 0.1058 for the no interbank case and 0.1008 for the interbank case).

\subsubsection{Communities}

At the beginning of each simulation we constructed four fixed communities of $M=20$ (maximum Chebyshev distance), and of size $3 M^{2}$, two of which are blocks of strictly patient individuals and two are strictly impatient individuals, as presented before in section 6.5.2. As hypothesized, having such communities changes the performance of banking in the society: it increased the consumption level along with decreasing the inequality between individuals for societies with banks compared to the case of no banks.

For a society with no banks, the average aggregate consumption level for the 50 simulated societies is 692,688 , which is significantly lower than that of a society with banks (average of 703,618) as well as that of the interbank case (an average of $700,416)$. In addition the Gini index for societies with no banks has an average of 0.176, significantly higher than that of banks case (average Gini index of 0.157) and the interbank case (Gini index of 0.158).

We find that with communities, societies with no banks fall into an inferior state represented by lower successful investments combined with higher wasteful investment that were prematurely liquidated. The total amount invested successful (that is not 
liquidated) in societies with no banks had an average of 205,727, significantly lower than that of societies with banks (an average of 224,828 for the no interbank case and 221,413 for societies with interbank). The total amount of investments liquidated in societies with no banks had an average of 50,077 (an average percentage of 20\% of total investments in the long term asset) significantly higher than that of societies with banks for the no interbank case (a value of 46,210 representing an average arctangent of $17 \%$ of total investments in the long term asset). While there is no significant difference between the case of no banks and the interbank case which reported an average value of 50,107 (an average of 18\%).

In our previous case of a balanced society we had higher consumption levels for societies with no banks and we explained the significantly lower consumption levels for societies with banks as what we referred to as the cost of banking. In our present case, we claim that there is still the cost of banking, yet the inferiority of the case of no banks in investments and liquidation, now that individuals can not easily find trading partners, dramatically affected the consumption level and economic efficiency beyond the cost of banking in the societies where banks are allowed to establish.

It is interesting to observe that as we impose shocks on homogeneous societies and as we increase the frequency of shocks from a low frequency of every 15 periods to a high frequency of every other period and then construct permanent communities, the aggregate consumption level for societies with no banks decreases significantly at each stage. Similarly the aggregate consumption level for individuals in societies having banks (both cases of interbank and no interbank) decreases significantly from homogeneous scenario to lower frequency shocks to shocks every other period, nevertheless there are no significant differences between the scenario with frequent shocks and permanent communities.

Accompanying the decrease in aggregate consumption in societies with banks, we observe that imposing shocks to societies with banks working in isolation resulted in a significantly higher accumulated bank reserves compared to the scenario of no 
shocks, yet no significant differences between the two scenarios of high frequency and low frequency shocks. Similarly, moving from high frequency shocks to permanent communities, there is a significant increase in the average accumulated bank reserves for the case of no interbank market. On the other hand for societies with banks operating under the interbank market we observe that the bank reserves increase as we impose shocks. There are significant differences between the scenario of homogeneous societies and that of low frequency shocks, as well as a significant differences comparing low frequency shocks scenario with the high frequency one with more accumulated reserves to the later one. And unlike the case of banks working in isolation there are no significant differences between the high frequency scenario and the communities scenario.

The same patterns hold if comparing the aggregate consumption added to the aggregate banks reserves for the interbank case. We observe significant reductions moving from homogeneous to low frequency shocks to high frequency, while no significant difference between high frequency and permanent communities. For the case of banks working in isolation, there is a substantial reduction in the aggregate consumption added to banks reserves moving from homogeneous to low frequency to high frequency shock, yet we witness a significant increase moving from high frequency shocks to the communities scenario.

On the other hand, on the economic efficiency of the system comparing the different societies structure, as expected moving from homogeneous societies to societies with periodical preference shocks to communities significantly alters the performance of our banking systems as well as performance of societies with no banks. In societies with banks we observe that there is no significant difference between the amount successfully invested in the capital asset in the two scenarios of homogeneous societies and that of low frequency shocks. Nevertheless moving from low frequency to high frequency and to communities significantly reduces the amount of successful investments. As to be expected that moving along the four different scenarios (homogeneous to low 
frequency to high frequency and finally to communities) causes a significant increase in the amount of prematurely liquidated investments in the productive technology.

In societies with banks forced to work in isolation as well as in societies having banks with an interbank market, we find that the amount successfully invested in the productive technology is significantly reduced moving from homogeneous to low frequency shocks scenario to high frequency shocks scenario. Interestingly as we switch from high frequency shocks to communities, the successful amount invested in the productive technology increases reaching as high as that in the homogeneous society scenario, with no significant differences in the interbank societies, and even significantly higher than the homogeneous scenario for the societies where banks are forced to work in isolation.

As for the economic waste expressed in terms of the amount prematurely liquidated of the investment in productive technology, we find in the case of interbank market a significant increase as we move along the homogeneous to low shocks frequency to high shocks frequency and finally to communities. While for case of banks working in isolation, there are no significant difference between the waste in the homogeneous scenario and the low frequency shocks one, and an significantly increasing waste moving between the rest of the scenarios (from low frequency shocks to high frequency and then to communities).

\subsubsection{Further trends}

Unlike the previous scenario of balanced societies, where the relationship between the total amount invested in the productive technology and the portion liquidated of this amount is positively correlated in the three cases of no banks, banks no interbank market and interbank market, imposing a form of correlated preferences in our society alters the shape of such relation.

In particular, in the low frequency shocks, the correlation coefficient in the case of no banks increases to 0.6282 (compared to 0.4609 in homogeneous societies). It 
also increases in the case of banks working in isolation to 0.8911 (compared to 0.7798 in homogeneous societies). In the case of interbank market we find a rather different pattern where such relationship reverses to a negative one with correlation coefficient of -0.0131. Imposing a higher frequency shocks reveals another different story where the correlation coefficient reduces to 0.2997 in the case of no banks while it increases in the case of banks working in isolation to a coefficient value of 0.927 , and a remarkable decrease in the case of interbank to a correlation coefficient of -0.578 .

On the other hand for permanent communities scenario, the correlation coefficient for the no banks case remain around its previous level of 0.2498 , an improvement in the case of banks working in isolation where the correlation coefficient is reduced to 0.2507, and finally for the interbank case the correlation coefficient is -0.0711 . Thus, it is only with the interbank case that we witness wise investments in the productive technology model, achieving its best performance in the case of high frequency shocks, and interestingly it is also the same scenario under which banks working in isolation delivers their worst performance.

As for the other set of relationships examined earlier (section 7.2.3) in the case of homogeneous societies, we find more or less the same trends in the case of correlated preference shocks except for these related to aggregate individuals consumption in the case of communities. Considering the relationship between consumption level and the Gini index in the permanent communities scenario, the correlation coefficient for the banking case indicates, unlike in the other scenarios, a strongly negative relation mimicking also that of the no banks case (correlation coefficient of -0.892 for no banks case, -0.885 for no interbank case and -0.882 for the interbank case).

Similarly the relationship between the consumption level and the percentage of individuals not in banks is altered to a negative relation in the communities case (-0.374 for the no interbank case and -0.532 for the interbank case). Finally the previously witnessed negative relation between the accumulated bank reserves and the aggregate individuals' consumption is altered to a positive relation in permanent 
Omneia R.H. Ismail - PhD Thesis - McMaster University - CES

communities scenario $(0.157$ for the no interbank case and 0.651 for the interbank case).

\subsection{Conclusions}

In this chapter we examined the effects of banks and the interbank market on social and economic welfare of the society. We illustrated that compared to what individuals can achieve in direct investments in society having no banks, introducing banks in a society resulted in the emergence of a new segment of individuals achieving more than what was feasible without them in terms satisfaction of liquidity desires. We called this segment the thrilled individuals and showed how the rise of this segment is accompanied by the rise of banking (in terms of banking coverage in the society), and is characterized by an accompanying rise of inequality in individual satisfaction.

We then moved to examine the more observable effects of banking in our societies. We divided banks effects into two category: these related to individuals consumption level and those related to long term investment.

For the consumption level, we measured the aggregate consumption of all individuals in the society and showed that introducing banks in a society actually reduced it if compared to that of societies with no banks. We argued that the difference represents the cost of banking, since adding the accumulated banks reserves to the individuals consumption in these societies compensated for the differences. In other words, introducing banks redirected consumption from individuals to bank owners. On the other hand we found that introducing banks results in less inequality in the consumption level between individuals in the society.

In a closer investigation to our model, it was found among other relations that the consumption level is strongly positively correlated with the rise of the inequality level in the case of societies with banks, unlike the case of societies where no banks were allowed to be established. Also, it was found that the aggregated level of individuals 
consumption is strongly negatively correlated with banks reserves level, the former is also positively correlated with the percentage of individuals in the society not joining banks.

As for the economic effects of banking, we demonstrated that banks introduction into society actually reduced the amount of successful investments in the productive technology, but nevertheless also reduced the total amount of economic waste suffered in the system measured by early liquidation of such investments.

We also examined that effects of banking and interbank markets in more involved society structures where we considered periodic correlated preference shocks (both cases of low and high frequency shocks), as well as the case of permanently established preference communities.

In imposing such structures of preference in the society, we found that individuals as well as banks well-being are dramatically affected and resulted in uncompensated losses. In particular imposing low frequency shocks resulted in uncompensated losses for societies with banks, where even after adding the aggregate banks reserves to the individuals consumption level does not amount to the consumption level in societies with no banks.

On the other hand as we introduce high frequency shocks, this effect works in reverse, where now the sum of the banks reserves and consumption level in societies with banks, unlike the previous scenario of low frequency shocks, is actually higher than the consumption level for societies with no banks. Interestingly, imposing further restrictions in individuals' capabilities in finding trading partners by constructing permanent preference communities introduces a further case of uncompensated losses. Societies with no banks and with permanent preference communities fall into an inferior state where the consumption level drops dramatically below the consumption level of societies with banks (that is, even without counting for bank reserves, the later consumption level is significantly higher).

On the other hand, for the economic efficiency of the system we find that imposing 
shocks dramatically affects investments, with a reduction in the amount of successful investments and an increase in the liquidation amount. As before, societies with no banks yielded the highest amount successfully invested in the productive technology as well as the highest amount prematurely liquidated. Moving to the scenario of communities alters our results, where the case of no banks falls into a rather inferior state having a lower amount successfully invested in the productive technology in addition to a higher liquidation value.

For all the cases combined, the case of the interbank market resulted, while not necessarily in the highest investment amount, in the wisest investments in the society measured by the correlation between the total investment value and the percentage of investments prematurely liquidated. On the other hand we demonstrated that the cases for which individuals suffer high frequency preference shocks banks provided a superior performance in the system in terms of consumption level enjoyed by individuals added to banks reserves and amount liquidated in the productive technology.

Further as individuals face inherited obstacles in finding trading partners as in the permanent communities scenario, banking provided a superior performance on all measures considered. 


\section{Chapter 8}

\section{Conclusions and Further Directions}

With our simple model we were able to demonstrate several important features. In contrast to traditional economic models, our construction does not rely on any a priori equilibrium or agreed upon strict structure. Rather, our model is a dynamical setup of agents using inductive reasoning to learn about their environment, form predictions and act upon them. Agents do not join a bank because they are unable to invest for themselves or because it is always better to be in a bank as "good equilibrium". Even though we demonstrated that once established, banks will include the majority of individuals, individuals had their different reasons (willingness and timing) for joining the banks. Some joined banks as they directly benefited from the higher payoff offered by the bank, while others learned that it is worse not joining the bank (by losing their trading partners). In other words, we introduced a model where banks are established endogenously.

We saw how banks tried to better learn about their depositors and their liquidity needs, and when given the chance established an interbank market to guard against individual liquidity shocks. In a interactive dynamical setup, our model demonstrated that frequent bank failures and eventual oligopolies are common features when banks are forced to act in isolation. The presence of interbank links leads to fewer failures and a less concentrated banking system. 
Nevertheless, we note that the banking system flourished with an interbank market when the general circumstances are favourable to banks, that is, relatively low payoff to depositors, intermediate reserve level, and high speed of learning and adaptation. As difficulties arise, the advantages gained under the interbank market diminished compared to the case of banks working in isolation.

As for banks and interbank effects on social and economic status, introducing banks in the society defined a new category of thrilled (or more-than-satisfied) individuals. We showed how under banks it was the only possible occasion to witness the emergence of such a category, where individuals got more satisfaction than what could have been achieved in a society with no banks. In all our cases where banks emerged, most individuals, each searching for his benefits and based on their learning and experiences, joined the bank at the end of our simulations. Nevertheless the effects of banks on the "collective welfare" varied considerably. In "normal situations" where the liquidity preferences in the society are randomly and uniformly distributed, the resulted collective welfare deteriorated. The aggregate consumption level and successful investments are significantly lower than in societies with no banks, and in a direct transformation of wealth the reduction in individuals consumption reflected a rise in banks wealth.

On the other hand, banks benefit society in terms of higher aggregate consumption level, higher capital investments and less economic waste, in cases where societies have more complicated preference structures than the simple uniformly randomly distributed preferences, such as societies with high frequency of correlated preference shocks or societies with permanently established preference communities. In such societies banks delivered a superior state of liquidity transformation, social welfare and economic status. Finally it is worth noting that cases where banks are allowed to interact in an interbank market always delivered more efficient investments than those of individual direct investments in terms of less economic waste measured by the percentage of capital investments liquidated prematurely. 
In addition to the interesting results of our simple model, we demonstrated how to establish the internal forces of the banking organization that we claimed earlier on to be lacking form the empirically based set of studies. It was the process of individuals uncertainties and learning that paved the way to a process of bank emergence and constituted the necessities for bank learning, as well as the forces for bank runs, and interbank establishment.

We emphasize that our model is an agent-based one. Thus with our simplified version of societies and assumptions on our individuals we supplied a possible explanation and a possible view on the system and the development of banking, among many possible explanations and views. Extensions and alternative mechanisms are always possible with such simplified models, such as considering a continuum of time points instead of the three time points division of each period for investments and withdrawal of deposits, considering banks as price makers instead of price takers by giving them the possibility of determining the deposit contract payoff, or considering competition between banks over individuals depositors, etc.

Among the many extensions we can foresee, the crucial next step in our point of view within our model is to examine the network itself. Mainly we emphasized the effect of the existence of such interbank network on our system, we did not examine the shape nor the type of such a network. For such extension, larger societies need to be considered, coupled with the possibility of multiple bank establishments at the same period in such large societies (as in our model we are restricted to the possibility of only one bank establishment at a time). Is our model or another version of it capable of delivering an interbank network that actually resembles a scale-free one? If that is the case, we can then use it to fulfill one of the main motivating goals of agent-based models in general, namely that of serving as a "petri dish" simulation of an actual banking system, upon which one can test the effects of many real life policies and interventions. But alas, we must leave this ambitious goal for future work. 


\section{Appendix A}

\section{Numerical Experiments}

\section{A.1 Banking system performance: interbank vs no interbank}

Values for the mean and standard deviation for the 50 independent simulations under the different parameter selections, comparing interbank case versus no interbank.

\begin{tabular}{l|l|l|l|r|r|}
\cline { 2 - 5 } \multicolumn{1}{c|}{ Base Case } & \multicolumn{2}{c|}{ No interbank } & \multicolumn{3}{c|}{ Interbank } \\
\cline { 2 - 6 } \multicolumn{1}{c|}{} & mean & s.d. & mean & s.d. \\
\hline$\%$ surviving banks & 0.1179 & 0.0640 & 0.1895 & 0.0771 \\
\hline Size of largest bank & 0.6409 & 0.2041 & 0.5083 & 0.1584 \\
\hline Bank coverage & 0.6858 & 0.1421 & 0.7265 & 0.1252 \\
\hline
\end{tabular}

\begin{tabular}{|l|l|l|r|r|r|}
\cline { 2 - 5 } \multicolumn{1}{c|}{ Higher payoff } & \multicolumn{2}{c|}{ No interbank } & \multicolumn{3}{c|}{ Interbank } \\
\cline { 2 - 6 } \multicolumn{1}{c|}{} & mean & s.d. & mean & s.d. \\
\hline \% surviving banks & 0.0566 & 0.0360 & 0.0576 & 0.0532 \\
\hline Size of largest bank & 0.7989 & 0.2093 & 0.8069 & 0.2168 \\
\hline Bank coverage & 0.5155 & 0.2913 & 0.3900 & 0.3370 \\
\hline
\end{tabular}

\begin{tabular}{l|l|l|l|r|r|}
\cline { 2 - 5 } \multicolumn{1}{c|}{ Lower payoff } & \multicolumn{2}{c|}{ No interbank } & \multicolumn{3}{c|}{ Interbank } \\
\cline { 2 - 6 } \multicolumn{1}{c|}{} & mean & s.d. & mean & s.d. \\
\hline \% surviving banks & 0.2195 & 0.1039 & 0.3132 & 0.1096 \\
\hline Size of largest bank & 0.4756 & 0.1518 & 0.3882 & 0.1131 \\
\hline Bank coverage & 0.7488 & 0.0188 & 0.7550 & 0.0126 \\
\hline
\end{tabular}


Omneia R.H. Ismail - PhD Thesis - McMaster University - CES

\begin{tabular}{|l|l|l|r|r|r|}
\cline { 2 - 6 } \multicolumn{1}{c|}{ Slow learning speed } & \multicolumn{2}{c|}{ No interbank } & \multicolumn{3}{c|}{ Interbank } \\
\cline { 2 - 6 } \multicolumn{1}{c|}{} & mean & s.d. & mean & s.d. \\
\hline$\%$ surviving banks & 0.0993 & 0.0575 & 0.1400 & 0.0937 \\
\hline Size of largest bank & 0.7167 & 0.2424 & 0.6285 & 0.2418 \\
\hline Bank coverage & 0.6574 & 0.1437 & 0.6854 & 0.1531 \\
\hline
\end{tabular}

\begin{tabular}{|l|l|l|r|r|}
\cline { 2 - 5 } \multicolumn{1}{c|}{ Moderate learning speed } & \multicolumn{3}{c|}{ No interbank } & \multicolumn{3}{c|}{ Interbank } \\
\cline { 2 - 6 } \multicolumn{1}{l|}{} & mean & s.d. & mean & s.d. \\
\hline \% surviving banks & 0.1104 & 0.0575 & 0.1575 & 0.0848 \\
\hline Size of largest bank & 0.6808 & 0.2243 & 0.5599 & 0.2238 \\
\hline Bank coverage & 0.6671 & 0.2031 & 0.7139 & 0.1045 \\
\hline
\end{tabular}

\begin{tabular}{|l|r|r|r|r|}
\cline { 2 - 5 } \multicolumn{1}{c|}{ No reserves } & \multicolumn{2}{c|}{ No interbank } & \multicolumn{3}{c|}{ Interbank } \\
\cline { 2 - 6 } \multicolumn{1}{l|}{} & mean & s.d. & mean & s.d. \\
\hline \% surviving banks & 0.1179 & 0.0640 & 0.1500 & 0.0822 \\
\hline Size of largest bank & 0.6409 & 0.2041 & 0.5840 & 0.2052 \\
\hline Bank coverage & 0.6858 & 0.1421 & 0.6877 & 0.1243 \\
\hline
\end{tabular}

\begin{tabular}{|l|l|l|r|r|r|}
\cline { 2 - 6 } \multicolumn{1}{c|}{ Higher reserves } & \multicolumn{2}{c|}{ No interbank } & \multicolumn{3}{c|}{ Interbank } \\
\cline { 2 - 6 } & mean & s.d. & mean & s.d. \\
\hline \% surviving banks & 0.1179 & 0.0640 & 0.1567 & 0.0870 \\
\hline Size of largest bank & 0.6409 & 0.2041 & 0.6077 & 0.2306 \\
\hline Bank coverage & 0.6858 & 0.1421 & 0.6734 & 0.1781 \\
\hline
\end{tabular}

\begin{tabular}{|c|c|c|c|c|}
\hline \multirow{2}{*}{ Low Frequency Shocks } & \multicolumn{2}{|c|}{ No interbank } & \multicolumn{2}{|c|}{ Interbank } \\
\hline & \begin{tabular}{|l|l} 
mean & s
\end{tabular} & s.d. & mean & s.d. \\
\hline$\%$ surviving banks & 0.1015 & 0.0617 & 0.1706 & 0.1035 \\
\hline Size of largest bank & 0.6989 & 0.2069 & 0.5859 & 0.2278 \\
\hline Bank coverage & 0.6998 & 0.1884 & 0.7351 & 0.1657 \\
\hline
\end{tabular}

\begin{tabular}{|c|c|c|c|c|}
\hline \multirow{2}{*}{ High Frequency shocks } & \multicolumn{2}{|c|}{ No interbank } & \multicolumn{2}{|c|}{ Interbank } \\
\hline & \begin{tabular}{|l|l} 
mean & $\mathrm{s}$
\end{tabular} & s.d. & mean & s.d. \\
\hline$\%$ surviving banks & 0.0599 & 0.0333 & \begin{tabular}{|l|}
0.0855 \\
\end{tabular} & 0.0567 \\
\hline Size of largest bank & 0.8140 & 0.1778 & 0.7711 & 0.2444 \\
\hline Bank coverage & 0.5871 & 0.3538 & 0.6736 & 0.3110 \\
\hline
\end{tabular}

\begin{tabular}{|l|r|r|r|r|}
\cline { 2 - 6 } \multicolumn{1}{c|}{ Communities } & \multicolumn{2}{c|}{ No interbank } & \multicolumn{3}{c|}{ Interbank } \\
\cline { 2 - 6 } \multicolumn{1}{c|}{} & mean & s.d. & mean & s.d. \\
\hline \% surviving banks & 0.2357 & 0.0919 & 0.2800 & 0.1064 \\
\hline Size of largest bank & 0.4666 & 0.1324 & 0.4855 & 0.4048 \\
\hline Bank coverage & 0.6760 & 0.0277 & 0.6700 & 0.0426 \\
\hline
\end{tabular}

\section{A.2 Effects of banking on social measures}

Values for the mean and standard deviation for the 50 independent simulations under the different scenarios (base case, periodical shocks and communities), comparing societies with banks (interbank and no interbank case) versus societies with no banks. 
Omneia R.H. Ismail - PhD Thesis - McMaster University - CES

\begin{tabular}{|l|r|r|r|r|r|r|}
\cline { 2 - 7 } \multicolumn{1}{c|}{ Base Case } & \multicolumn{2}{c|}{ No interbank } & \multicolumn{2}{c|}{ Interbank } & \multicolumn{2}{c|}{ No Banks } \\
\cline { 2 - 7 } \multicolumn{1}{c|}{} & mean & s.d. & mean & s.d. & mean & s.d. \\
\hline Successful long term Investment & 222524.699 & 1892.189 & 219966.692 & 3324.999 & 224795.280 & 2636.456 \\
\hline Investments liquidated & 26952.454 & 1896.011 & 28863.357 & 2123.504 & 31326.320 & 759.795 \\
\hline Consumption & 716708.727 & 1936.637 & 716758.498 & 2747.420 & 721132.120 & 2381.792 \\
\hline Gini index & 0.117 & 0.004 & 0.116 & 0.005 & 0.129 & 0.001 \\
\hline Banks' reserves & 4339.745 & 1644.957 & 4768.650 & 2136.571 & - & - \\
\hline \% invetsment liquidated & 0.108 & 0.006 & 0.116 & 0.007 & 0.122 & 0.002 \\
\hline Reserves+consumption & 721048.472 & 1708.596 & 721527.148 & 2199.644 & 721132.120 & 2381.792 \\
\hline
\end{tabular}

\begin{tabular}{|c|c|c|c|c|c|c|}
\hline \multirow[t]{2}{*}{ Low Frequency Shocks } & \multicolumn{2}{|c|}{ No interbank } & \multicolumn{2}{|c|}{ Interbank } & \multicolumn{2}{|c|}{ No Banks } \\
\hline & mean & s.d. & mean & s.d. & mean & s.d. \\
\hline Successful long term Investment & 220297.993 & 2994.681 & 215915.981 & 4360.232 & 224116.340 & 2311.646 \\
\hline Investments liquidated & 27431.273 & 3225.951 & 31507.178 & 1568.415 & 32890.060 & 925.801 \\
\hline Consumption & 712803.620 & 4016.196 & 712940.194 & 3663.816 & 719671.310 & 2006.804 \\
\hline Gini index & 0.118 & 0.107 & 0.114 & 0.008 & 0.128 & 0.001 \\
\hline Banks' reserves & 5778.736 & 3154.618 & 6017.487 & 3412.174 & - & - \\
\hline$\%$ invetsment liquidated & 0.111 & 0.011 & 0.127 & 0.006 & 0.128 & 0.002 \\
\hline Reserves+consumption & 718582.357 & 2184.013 & 718957.682 & 1562.927 & 719671.310 & 2006.804 \\
\hline
\end{tabular}

\begin{tabular}{|l|r|r|r|r|r|r|}
\cline { 2 - 7 } \multicolumn{1}{c|}{ High Frequency shocks } & \multicolumn{2}{c|}{ No interbank } & \multicolumn{2}{c|}{ Interbank } & \multicolumn{2}{c|}{ No Banks } \\
\cline { 2 - 7 } & \multicolumn{1}{|c|}{ mean } & S.d. & mean & s.d. & mean & s.d. \\
\hline Successful long term Investment & 213606.871 & 2611.657 & 199552.626 & 13080.147 & 215224.780 & 2542.005 \\
\hline Investments liquidated & 34497.355 & 5392.824 & 44630.977 & 6888.948 & 40541.620 & 1027.169 \\
\hline Consumption & 701537.753 & 4708.852 & 699024.106 & 5268.009 & 706953.970 & 2339.739 \\
\hline Gini index & 0.106 & 0.010 & 0.101 & 0.012 & 0.118 & 0.001 \\
\hline Banks' reserves & 6820.440 & 5688.949 & 9301.808 & 6210.972 & - & - \\
\hline$\%$ invetsment liquidated & 0.139 & 0.018 & 0.183 & 0.032 & 0.158 & 0.003 \\
\hline Reserves+consumption & 708358.194 & 2614.875 & 708325.914 & 2813.305 & 706953.970 & 2339.739 \\
\hline
\end{tabular}

\begin{tabular}{|c|c|c|c|c|c|c|}
\hline \multirow{2}{*}{ Communities } & \multicolumn{2}{|c|}{ No interbank } & \multicolumn{2}{|c|}{ Interbank } & \multicolumn{2}{|c|}{ No Banks } \\
\hline & \begin{tabular}{|l|l|} 
mean \\
\end{tabular} & s.d. & mean & s.d. & mean & s.d. \\
\hline Successful long term Investment & 224828.720 & 9342.642 & 221413.388 & 11807.823 & \begin{tabular}{|l|l|}
3 & 205727.400 \\
\end{tabular} & 9427.988 \\
\hline \begin{tabular}{|l} 
Investments liquidated \\
\end{tabular} & 46210.532 & 9723.250 & 50107.103 & 10868.912 & 50077.400 & 9917.826 \\
\hline Consumption & 703618.902 & 13326.096 & 700416.445 & 15390.624 & 692688.700 & 14199.614 \\
\hline Gini index & 0.157 & 0.009 & 0.158 & 0.010 & 0.176 & 0.008 \\
\hline Banks' reserves & 10104.553 & 1860.474 & 9944.752 & 1950.121 & - & \\
\hline \% invetsment liquidated & 0.170 & 0.035 & 0.185 & 0.040 & 0.196 & 0.038 \\
\hline Reserves+consumption & 713723.455 & 13741.213 & 710361.198 & 16726.254 & 692688.700 & 14199.614 \\
\hline
\end{tabular}




\section{Bibliography}

[AB00] R. Albert and A. L. Barabási. Topology of evolving networks: local events and universality. Physical Review Letters, 85(24), December 2000.

[AB02] R. Albert and A. L. Barabási. Statistical mechanics of complex networks. Review of Modern Physics, 74:47-97, January 2002.

[AB08] F. Allen and A. Babus. Networks in finance. Wharton Financial Institutions Center Working Paper, (08-07), August 2008.

[AB09] R. Albert and A. L. Barabási. The Geometry of Discrete Groups. A Plume Book, New York, 2009.

[AG98] F. Allen and D. Gale. Financial contagion. Wharton School Working Paper, (98-31), October 1998.

[AJB00] R. Albert, H. Jeong, and A. L. Barabási. Error and attack tolerance of complex networks. Nature, 406:378382, 2000.

[Art92] W. Brian Arthur. On learning and adaptation in the economy. Santa Fe Institute Working Paper, 92-07-038, May 1992.

[Art94] W. Brian Arthur. Complexity in economic theory: Inductive reasoning and bounded rationality. The American Economic Review, 84(2):406-411, May 1994. 
Omneia R.H. Ismail - PhD Thesis - McMaster University - CES

[Atk70] A. B. Atkinson. On the measurement of inequality. Journal of Economic Theory, 2:244-263, 1970.

[BA99] A. L. Barabás and R. Albert. Emergence of scaling in random networks. Science, 286(5439):509-512, October 1999.

[Bab07] A. Babus. The formation of financial networks. Fondazione eni enrico mattei Working Paper, (129), 2007.

[BBCR03] B. Bollobás, C. Borgs, J. Chayes, and O. Riordan. Directed scale-free graphs. Proceedings of the Fourteenth Annual ACM-SIAM Symposium on Discrete Algorithms, pages 132-139, 2003.

[BEST04] M. Boss, H. Elsinger, M. Summer, and S. Thurner. Network topology of the interbank market. Quantitative Finance, 4:677-684, December 2004.

[BGH89] L. B. Booker, D.E. Goldberg, and J.H. Holland. Classifier system and genetic algorithm. Artificial Intelligence, 40(1-3):235-282, September 1989.

[Cal07] G. Caldarelli. Scale-Free Networks, complex webs in nature and technology. Oxford University Press, New York, 2007.

$\left[\mathrm{CFH}^{+} 08\right]$ D. Colander, H. Follmer, A. Haas, M. Goldberg, K. Juselius, A. Kirman, T. Lux, and B. Sloth. The financial crisis and the systemic failure of academic economics. Opinion Paper Following $98^{\text {th }}$ Dahlem Workshop, 2008.

[CFS05] R. Cifuentes, G. Ferrucci, and H. S. Shin. Liquidity risk and contagion. Journal of European economic association, pages 556-566, 2005.

[CG91] C. Calomiris and G. Gorton. The origins of banking panics, models, facts, and bank regulation. pages 109-174, 1991. 
Omneia R.H. Ismail - PhD Thesis - McMaster University - CES

[CLM04] P. Crucitti, V. Latora, and M. Marchiori. Model for cascading failures in complex networks. Phys. Rev. E, 69, 2004.

[CLMR04] P. Crucitti, V. Latora, M. Marchiori, and A. Rapisarda. Error and attack tolerance of complex networks. Physica A, 340:388-394, 2004.

[CS09] R. Cont and E. B. Santos. Banking system topology and systemic risk: the brazilian network empirical analysis. Working Paper, September 2009.

[CSN09] A. Clauset, C. R. Shalizi, and M. E. J. Newman. Power-law distributions in empirical data. SIAM review, 51(4):661-703, 2009.

[CT07] D. O. Cajueiro and B. M. Tabak. The role of banks in the brazilian market; does bank type matter. Banco Central Do Brasil Working Paper Series, (130), January 2007.

[Das04] A. Dasgupta. Financial contagion through capital connections: a model of the origin and spread of bank panics. Journal of the European Economic Association, 2, December 2004.

[DD00] D. W. Diamond and P. H. Dybvig. Bank runs, deposit insurance and liquidity. Federal reserve bank of Minneapolis Quartely Review, 24(1):1423, Winter 2000.

[Dia07] D. W. Diamond. Banks and liquidity creation: a simple exposition of the diamond-dybvig model. Economic Quarterly, 2007.

[DJW98] Steven H. Strogatz Duncan J. Watts. Collective dynamics of small-world networks. Nature, 393(6684):440-442, June 1998.

[DN07] H. Degryse and G. Nguyen. Interbank exposures: an empirical examination of contagion risk in the belgian banking sector. International Journal of Central Banking, 3(2), June 2007. 
[Fer08] N. Ferguson. The Ascent of Money: A Financial History of the World. Penguin, New York, 2008.

[Fri61] M. Friedman. Capitalism and freedom. University of Chicago Press, Chicago, 1961.

[FS02] X. Freixas and A M Santomero. An overall perspective on banking regulatio. Federal Reserve Bank of Philadelphia Working Papers, (02-1), February 2002 .

[GB08] T. Gross and B. Blasius. Adaptive coevolutionary networks: a review. J. R. Soc. Interface, 5:259-271, 2008.

[GJK07] P. Gai, N. Jenkinson, and S. Kapadia. Systemic risk in modern financial systems: analytics and policy design. Journal of Risk Finance, 8(2):156$165,2007$.

[Hal09] A. G. Haldane. Rethinking the financial network. Speech Delivered at the Financial Student Association, Amsterdam, April 2009.

[Hay44] F. A. Hayek. The Road to Serfdom. University of Chicago Press, Chicago, 1944.

[HC00] P. Howitt and R. Clower. The emergence of economic organization. Journal of Economic Behavior \&3 Organization, 41:55-84, 2000.

[How08] P. Howitt. Macroeconomics with intelligent autonomous agents. In Macroeconomics in the Small and Large: Essays on Microfoundations, Macroeconomics Applications and Economic History in Honor of Axel Leijonhufvud, pages 157-177, 2008. edited by Roger Farmer. Cheltenham: Edward Elgar.

[IJP06] G. Iori, S. Jafarey, and F. G. Padilla. Systemic risk on the interbank market. Journal of Economic Behavior \& Organization, 61:525-542, 2006. 
[INST04] H. Inaoka, T. Ninomia, T. Shimizu, and H. Takayasu. Fractal network derived from banking transaction- an anlysis of network structures formed by financial institutions. Bank of Japan Working Paper Series, (04), April 2004.

[JT96] Rochet J.C and J. Tirole. Interbank lending and systemic risk. Journal of Money, Credit and Banking, 28(2):733-762, November 1996.

[Kah11] D. Kahneman. Thinking fast and slow. Doubleday, Canada, 2011.

[LL04] I. Lelyveld and F. Liedorp. Interbank contagion in the dutch banking sector. MPRA, October 2004.

[LM09] C. Lazzetta and M. Manna. The topology of the interbank market: developments in italy since 1990. Banca D'Italia Working Papers, (711), May 2009.

[MBB99] W. Mendenhall, R. Beaver, and B. Beaver. Introduction to probability and statistics. Duxbury Press, 1999.

[MH04] B. Mandelbrot and R. Hudson. The (mis)bahavior of markets, a fractal view of financial turbulence. New York: Basic Books, New York, 2004.

[Mit09] M. Mitchel. Complexity, A guided tour. Oxford university press, New York, 2009.

[ML02] A. Motter and Y. C. Lai. Cascade-based attacks on complex networks. Phys. Rev. E, 66, 2002.

[MLS08] R. M. May, S. A. Levin, and G. Sugihara. Ecology for bankers. Nature, 451:893895, 2008.

[New10] M.E.J. Newman. Networks, an introduction. Oxford university press, New York, 2010. 
Omneia R.H. Ismail - PhD Thesis - McMaster University - CES

[NYYA07] E. Nier, J. Yang, T. Yorulmazer, and A. Alentorn. Network models and financial stability. Journal of Economic Dynamics $\mathscr{E}$ Control, pages 20332060, March 2007.

[Oor90] C. J. Oort. Banks and the stability of the international financial system. De Economist, 138(4), 1990.

[Rom09] P. P. Romero. Essays on banking and capital: an agent-based investigation. PhD thesis, George Mason University, 2009.

$\left[\mathrm{SBA}^{+} 06\right]$ K. Soramaki, M. Bech, J. Arnold, R. Glass, and W. Beyeler. The topology of the interbank payment flows. FRBNY Staff Rep, (243), March 2006.

[Sen76] A. Sen. Poverty: An ordinal approach to measurement. Econometica, 44:219-231, 1976.

[Shi08] R. Shiller. The subprime solution. Princeton University Press, 2008.

[Sim55] Herbert A. Simon. A behavioral model of rational choice. Quarterly Journal of Economics, 69(1):99-118, 1955.

[SLU09] S.J.Goerner, B. Lietaer, and R.E. Ulanowicz. Quantifying economics sustainability: implications for free-enterprise theory, policy and practice. Ecological Economics, 69:76-81, 2009.

[SP09] Standards and Poor's. Global credit portal, ratingsdirect, default, transition, and recovery: 2009 annual global corporate default study and rating transitions. 2009.

[Str01] S. H. Strogatz. Exploring complex networks. Nature, 410, March 2001.

[Tem97] T. Temzelides. Evolution, co-ordination and banking panics. Journal of Monetary Economics, 40:163-183, 1997. 
[Wat02a] D. J. Watts. A simple model of global cascades on random networks. PNAS, 99(9):5766-5771, April 2002.

[Wat02b] Duncan J. Watts. Six degrees. W. W. Norton \& Company, New York, London, 2002.

[Wil27] E. B. Wilson. Probable inference, the law of succession, and statistical inference. Journal of the American Statistical Association, 22(158):209$212,1927$. 